\title{
Composition in Distributional Models of
}

\section{Semantics}

Jeffrey Mitchell

Doctor of Philosophy

School of Informatics

University of Edinburgh

2011 


\section{Abstract}

Distributional models of semantics have proven themselves invaluable both in cognitive modelling of semantic phenomena and also in practical applications. For example, they have been used to model judgments of semantic similarity (McDonald, 2000) and association (Denhire and Lemaire, 2004; Griffiths et al., 2007) and have been shown to achieve human level performance on synonymy tests (Landuaer and Dumais, 1997; Griffiths et al., 2007) such as those included in the Test of English as Foreign Language (TOEFL). This ability has been put to practical use in automatic thesaurus extraction (Grefenstette, 1994). However, while there has been a considerable amount of research directed at the most effective ways of constructing representations for individual words, the representation of larger constructions, e.g., phrases and sentences, has received relatively little attention. In this thesis we examine this issue of how to compose meanings within distributional models of semantics to form representations of multi-word structures.

Natural language data typically consists of such complex structures, rather than just individual isolated words. Thus, a model of composition, in which individual word meanings are combined into phrases and phrases combine to form sentences, is of central importance in modelling this data. Commonly, however, distributional representations are combined in terms of addition (Landuaer and Dumais, 1997; Foltz et al., 1998), without any empirical evaluation of alternative choices. Constructing effective distributional representations of phrases and sentences requires that we have both a theoretical foundation to direct the development of models of composition and also a means of empirically evaluating those models.

The approach we take is to first consider the general properties of semantic composition and from that basis define a comprehensive framework in which to consider the composition of distributional representations. The framework subsumes existing proposals, such as addition and tensor products, but also allows us to define novel composition functions. We then show that the effectiveness of these models can be 
evaluated on three empirical tasks.

The first of these tasks involves modelling similarity judgements for short phrases gathered in human experiments. Distributional representations of individual words are commonly evaluated on tasks based on their ability to model semantic similarity relations, e.g., synonymy or priming. Thus, it seems appropriate to evaluate phrase representations in a similar manner. We then apply compositional models to language modelling, demonstrating that the issue of composition has practical consequences, and also providing an evaluation based on large amounts of natural data. In our third task, we use these language models in an analysis of reading times from an eye-movement study. This allows us to investigate the relationship between the composition of distributional representations and the processes involved in comprehending phrases and sentences.

We find that these tasks do indeed allow us to evaluate and differentiate the proposed composition functions and that the results show a reasonable consistency across tasks. In particular, a simple multiplicative model is best for a semantic space based on word co-occurrence, whereas an additive model is better for the topic based model we consider. More generally, employing compositional models to construct representations of multi-word structures typically yields improvements in performance over non-compositonal models, which only represent individual words. 


\section{Acknowledgements}

I am deeply grateful to my supervisor, Mirella Lapata, for her guidance, criticism and insight. The substance and detail of the work presented here owes much to her input. I would also like to thank Victor Lavrenko, Steve Renals and Paola Merlo, my second supervisor and examiners, who provided fresh viewpoints and stimulating discussions. In addition, the feedback from and discussions with numerous other researchers has been greatly appreciated. Finally, my debt to friends and family, for their support and encouragement, has to be acknowledged. Thank you. 


\section{Declaration}

I declare that this thesis was composed by myself, that the work contained herein is my own except where explicitly stated otherwise in the text, and that this work has not been submitted for any other degree or professional qualification except as specified.

(Jeffrey Mitchell) 


\section{Table of Contents}

1 Introduction 1

1.1 Composition in Distributional Models . . . . . . . . . . . . . 1

1.2 Contributions ......................... 4

1.3 Thesis Structure . . . . . . . . . . . . . . . 5

1.4 Publications ........................ 8

2 Background 9

2.1 Theories and Models of Semantics . . . . . . . . . . . . . . 9

2.2 Symbolic and Non-symbolic Representations . . . . . . . . . . . 13

2.3 Semantic Composition . . . . . . . . . . . . . 20

2.4 Distributional Semantics . . . . . . . . . . . . . . . 25

2.4.1 Composition in Distributional Models . . . . . . . . . . 33

2.5 Conclusions . . . . . . . . . . . . . . . . 35

3 Constructing Distributional Representations of Word Meaning 37

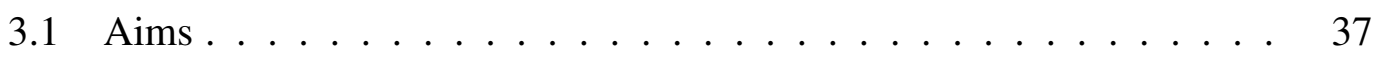

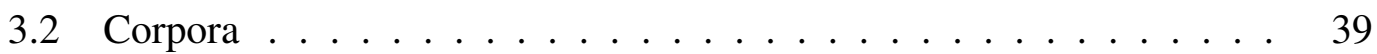

3.3 Acquiring Distributional Counts . . . . . . . . . . . . . . 40

3.3.1 Simple Semantic Space . . . . . . . . . . . . . . 41

3.3.2 Latent Dirichlet Allocation . . . . . . . . . . . . . . 41

3.4 Defining the Space . . . . . . . . . . . . . . 43

3.5 Evaluation . . . . . . . . . . . . . . . . . 47 
3.5.1 Predicting Similarity Judgements _ . . . . . . . . . . . 47

3.5.2 Identifying Synonyms . . . . . . . . . . . 51

3.5.3 Discussion ..................... 54

3.6 Conclusions . . . . . . . . . . . . . . . . 55

4 A Framework for Vector Composition $\quad 57$

4.1 Preliminaries ...................... 57

4.2 Composition Functions $\ldots \ldots$. . . . . . . . . . . 60

4.3 Conclusions . . . . . . . . . . . . . . . . . . . 69

5 Modeling Phrase Similarity $\quad 70$

5.1 Methodology ..................... 70

5.2 Experiment $1 \ldots \ldots \ldots \ldots \ldots$

5.2.1 Materials and Design . . . . . . . . . . . . . 74

5.2.2 Procedure and Subjects . . . . . . . . . . . . . 75

5.2.3 Model Parameters . . . . . . . . . . . . . . . 76

5.2 .4 Results . . . . . . . . . . . . . . 77

5.2 .5 Discussion .................... 79

5.3 Experiment $2 \ldots \ldots \ldots \ldots$. . . . . . . . . . . 80

5.3.1 Materials and Design . . . . . . . . . . . . . 82

5.3.2 Procedure and Subjects . . . . . . . . . . . . . . 83

5.3.3 Model Parameters . . . . . . . . . . . . . . . . . . 84

5.3 .4 Results .................... 85

5.3 .5 Discussion ..................... 90

5.4 Conclusions . . . . . . . . . . . . . . . . . . . 92

6 Language models based on Vector Composition 94

6.1 Language models . . . . . . . . . . . . . . . . . . . . 96

6.1 .1 Syntactic Models . . . . . . . . . . . . . . 97

6.1 .2 Ngram Models . . . . . . . . . . . . . . . . . . . . . 99 
6.1.3 Semantic Models . . . . . . . . . . . . . . . . . 101

6.1.4 Connectionist Language Models . . . . . . . . . . . . . . 104

6.2 Vector Composition . . . . . . . . . . . . . . . . . . 106

6.2.1 Vector Composition from a Probabilistic Perspective . . . . . 107

6.2.2 Deriving a Language Model . . . . . . . . . . . . . . . 109

6.2.3 Integrating with Other Language Models . . . . . . . . . . . 110

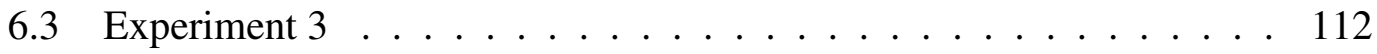

6.3 .1 Method .................... 112

6.3 .2 Data......................... 114

6.3.3 Model Parameters . . . . . . . . . . . . . . 114

6.3 .4 Results ....................... 115

6.3 .5 Discussion .................... 118

6.4 Conclusions . . . . . . . . . . . . . . . . . . . 119

7 Predicting Eye-movements in Reading 121

7.1 Cognitive Processes and Eye-movements in Reading . . . . . . . . 122

7.1.1 Eye-movements . . . . . . . . . . . . . . 122

7.1 .2 Cognitive Load . . . . . . . . . . . . . . . . . . 124

7.2 Surprisal for Compositional Language Models . . . . . . . . . . . . 129

7.3 Experiment $4 \ldots \ldots \ldots \ldots \ldots \ldots$

7.3.1 Analysis Methodology . . . . . . . . . . . . . . 132

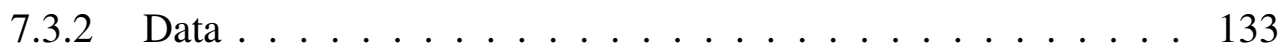

7.3 .3 Results . . . . . . . . . . . . . . 135

7.3 .4 Discussion . . . . . . . . . . . . . . . 138

7.4 Conclusions . . . . . . . . . . . . . . . . . . . . . . 139

8 Conclusions 140

8.1 Aims of the Thesis . . . . . . . . . . . . . . 140

8.2 Summary of Contributions . . . . . . . . . . . . . . 141 
8.3 General Discussion . . . . . . . . . . . . . . . . . . . 144

8.4 Future Work . . . . . . . . . . . . . . . . . . . . 147

A Simple Vector and Tensor Algebra 152

B Instructions for Experiment 1 156

$\begin{array}{ll}\text { C Materials for Experiment 1 } & 158\end{array}$

$\begin{array}{llr}\text { D Instructions for Experiment 2 } & 161\end{array}$

$\begin{array}{llr}\text { E } & \text { Materials for Experiment 2 } & 163\end{array}$

$\begin{array}{lr}\text { Bibliography } & 172\end{array}$ 


\section{Chapter 1}

\section{Introduction}

This chapter introduces the problem of composition in distributional models of semantics, details our contributions and outlines the structure of the rest of the thesis.

\subsection{Composition in Distributional Models}

Distributional methods, which allow semantic representations to be constructed from the patterns of word usage in large corpora, have proven themselves effective both in modelling cognitive phenomena and also in practical applications. For example, they have been used to model human similarity judgements (McDonald, 2000), enhance $n$-gram language models with long range semantic information (Bellegarda, 2000; Coccaro and Jurafsky, 1998) and quantify the effect of semantic constraint on reading times (Pynte et al., 2008). The basic idea is that words with similar meanings will be found in similar contexts, and that therefore the way in which a word's occurrences are distributed across a set of contexts can be used to infer its meaning (Firth, 1957; Harris, 1954). Practical implementations of this idea range from ad-hoc approaches for turning word co-occurrence statistics into vector based representations (Lund and Burgess, 1996) to sophisticated generative models of the distribution of words across the documents in a corpus (Blei et al., 2003).

However, these models are typically directed at the representation of isolated words, 
as opposed to phrases or sentences. The basic representations are vectors which capture the pattern of distribution of single words across a set of contexts, and evaluation of these representations is commonly based on relations of semantic similarity between individual words, e.g., identifying synonyms (Landuaer and Dumais, 1997; Griffiths et al., 2007) or modelling semantic priming (Lund and Burgess, 1996; Landuaer and Dumais, 1997). In the latter task, recognition of a word is facilitated by its being preceded by another word with a similar meaning, and the fact that distributional representations can be used to predict this effect demonstrates their relevance as models for the access and processing of lexical semantic information in cognition. While considerable research has been directed at optimising the construction of these word level representations (e.g., Bullinaria and Levy, 2007; Weeds, 2003; Curran, 2003), less attention has been focused on the question of how to combine them. A common method has been vector addition (Landauer et al., 1997; Foltz et al., 1998; Coccaro and Jurafsky, 1998), but this is unsatisfactory for a number of reasons. Firstly, without a theoretical motivation or an empirical evaluation of alternatives, there is little reason to believe that addition effectively models the way in which meanings combine. Secondly, addition is symmetric and so takes no account of syntax or word order, meaning it is essentially a bag-of-words approach. Together, these criticisms suggest that while additive representations may capture important semantic information about the collection of individual words within a larger construction, they probably fail to capture the full meaning that derives from the interaction of those words within their syntactic structure. In contrast, much experimental evidence suggests that semantic similarity is more complex than simply a relation between isolated words. For example, Duffy et al. (1989) showed that priming of sentence terminal words was dependent not simply on individual preceding words but on their combination, and Morris (1994) later demonstrated that this priming also showed dependencies on the syntactic relations in the preceding context.

The dependency of the process of semantic composition on syntactic structure can 
be elegantly modelled in terms of representations based on symbolic logic (Montague, 1974; Blackburn and Bos, 2005). In this approach, the composition of a modifier with a head, for example, can be modelled in terms of the application of a function, representing the modifier, to an argument, representing the head, to produce a result representing the semantics of the modified head. These functions are expressed in terms of the lambda calculus, and this allows a tight correspondence between syntactic types and functional types to be defined. While some work (Clark et al., 2008) has investigated the theoretical possibility of applying this approach to distributional models, the practical details of implementation and evaluation are lacking. In particular, the question of what sort of function is required to combine the constituent representations remains open.

As an alternative to simple vector addition, one set of approaches (Aerts and Czachor, 2004; Clark and Pulman, 2007; Widdows, 2008) has proposed using vector binding operations (Smolensky, 1990; Plate, 1991). The intention here is to use concatenation of vectors to build up structured representations in a way that emulates symbolic approaches. In contrast, the approach of Kintsch (2001) builds on an existing model (Kintsch, 1988) of how information is integrated during comprehension. In both cases, evaluation has been weak, either being absent or only relying on small numbers of hand-picked examples.

There is therefore a strong motivation to find a solid basis on which to investigate the issue of composition in distributional models of semantics, and also to develop robust evaluation paradigms of proposed composition operations. Our approach is to first develop a framework for considering the problem, based on a general discussion of the nature of semantic composition. We then relate the existing approaches to the framework, and also introduce novel proposals. Following that, we show that these models can be evaluated on three tasks using substantial quantities of natural data. The first task involves predicting similarity ratings for short phrases and allows us to test which models are most effective at modelling the semantic relations perceived 
by experimental subjects. The second task investigates whether these relations are relevant to the semantic structure present in a corpus of news data, by exploiting them in a compositional language model. Finally, our third task relates the predictions made by this model to the semantic expectations of readers, in terms of a regression model for reading times derived from an eye-tracking study.

\subsection{Contributions}

Modeling Our work makes novel contributions to the modeling of semantic composition both in terms of the operation of composition itself and also in terms of the use of compositional representations in modeling the semantic dependencies within natural text. We introduce a framework for composition in Chapter 4, which allows us to compare existing proposals and identify their differences and similarities. This leads us to consider the constraints and assumptions that can be used to derive functions within this framework. As a result, we propose three novel approaches: the simple multiplicative, weighted addition and dilation models. We then derive a language model that exploits the semantic relations between a word and its history using representations produced by such compositional models. This is also combined with an $n$-gram model and a probabilistic parser, to produce a language model that integrates lexical, syntactic and semantic dependencies. Finally, we derive a surprisal based measure of processing load from this integrated model.

Evaluation We develop three novel methods of evaluating compositional models based on substantial quantities of natural text. For the first of these, we collect a large dataset of similarity ratings for short phrases from native English speakers and correlate these judgements with the predictions of our compositional models. We then evaluate the perplexity of the semantic language models derived from these representations as a means of quantifying how well they capture semantic dependencies in news data. Finally, a regression of reading times against the corresponding surprisal mea- 
sures reveals the relevance of these dependencies to the semantic relations perceived by readers.

Findings Our experiments produce a number of noteworthy results. On all three tasks we find that there is a dependence between the structure of the underlying semantic representations and the form of the composition model. Specifically, the simple additive model tends to produce the best results for representations based on Latent Dirichlet Allocation (Blei et al., 2003), whereas our novel simple multiplicative model is more effective on the simple semantic space representations. On the phrase similarity task, the existing proposals are outperformed by our novel proposals: the simple multiplicative, weighted addition and dilation models. In particular, circular convolution (Widdows, 2008; Plate, 1991), a vector binding function, gives very weak results. This eliminates the possibility that effective models of complex semantic structures can be constructed by simply binding together distributional representations of the constituents. We also find that the semantic dependencies in natural text modelled by our compositional representations make significant contributions to language modelling and predicting processing difficulty in reading.

\subsection{Thesis Structure}

In overview, we will first set up the foundations of the thesis in Chapters 2, 3 and 4, where we will cover the relevant background topics, construct our basic distributional models and introduce a framework for considering composition in these models. We will then carry out our emprirical evaluations of the compositional models in Chapters 5, 6 and 7, with experiments testing the ability of these models to predict similarity judgements for short phrases, enhance $n$-gram language models with long range semantic dependencies and predict eye-movements in reading. Finally, Chapter 8 will summarise the conclusions to be drawn from this work and suggest future directions.

In more detail, Chapter 2 presents an overview of the main concepts and issues 
relevant to this thesis. This discussion starts with the questions of what meaning is and how it may be represented. The contrast between logical and distributional models of semantics leads to a more general discussion of different approaches to representation. In particular, we describe the vector binding operations that connectionist researchers proposed would allow them to emulate the structures of symbolic representations. We then turn our attention to the nature of semantic composition, and cover attempts to characterise both what it is and what it is not. Finally, we consider distributional models in more detail, examining their motivations and implementations, and describe the existing approaches to composing these representations including the aformentioned vector binding operations.

Following that discussion, a range of semantic models are constructed and evaluated in Chapter 3. Within two broad approaches, a simple semantic space and a Latent Dirchlet Allocation (Blei et al., 2003) model, we consider various parameter settings and evaluate the resulting representations on two tasks. The first task involves predicting similarity ratings for pairs of words, whereas the second task requires the identification of synonyms from among a set of alternatives. Based on robust performance across these tasks, we choose a pair of models which will be used as a basis for composition in further experiments.

However, before proceeding to those experiments, we outline a framework for composition in distributional models in Chapter 4. Drawing on the discussion of the general nature of semantic composition in Section 2.3, this framework assumes that the composition of a pair of constituents is a function of those constituents, their syntactic relation, plus any addition background knowledge that is required. We relate a number of existing proposals, such as vector addition, circular convolution (Widdows, 2008; Plate, 1991), and Kintsch's (2001) model, to our framework and also develop a number of novel functions, such as the simple multiplicative and dilation models.

We then show that the putative composition functions can be evaluated on three tasks based on substantial quantities of natural data. In Chapter 5 we construct a large 
dataset of similarity ratings for short phrases, which we use to assess our compositional models. We consider subject-verb, adjective-noun, noun-noun and verb-object constructions, deriving our materials from real examples attested in the BNC. The ratings are collected from native English speakers, and the models are evaluated on their ability to predict these human judgements. Our results show that many of our novel proposals outperform existing approaches, with the multiplicative model on a simple semantic space producing the best performance.

Following that, we investigate the use of these compositional representations to capture semantic dependencies for language modelling in Chapter 6. For reasons of simplicity and efficiency we compare two syntax independent approaches to composition, the simple additive and simple multiplicative models, and this allows us to define an incremental compositional language model which uses semantic coherence to assign probabilities to upcoming words given their history. Integrating this semantic component with an $n$-gram and a syntactic model allows us to investigate the ability of this model to exploit long range semantic dependencies not captured by the other models, and we evaluate the results in terms of perplexity on a test set.

Chapter 7 takes this integrated language model and uses it to derive a measure of processing difficulty for reading times. Our approach is based on the notion of surprisal (Hale, 2001), which assumes that input which conflicts with readers expectations is associated with increased cognitive load. In a regression analysis on the Dundee eyetracking corpus, we find that the semantic, syntactic and $n$-gram components of the integrated surprisal measure are all significant predictors of reading time.

Finally, in Chapter 8, we review our findings, draw conclusions and outline directions for future work. 


\subsection{Publications}

The research underlying this thesis also formed the basis for a number of journal and conference publications. Much of the material in Chapters 4 and 5 was previously published in Mitchell and Lapata (2008) and Mitchell and Lapata (2010), with the former covering Experiment 1 and the latter Experiment 2. Mitchell and Lapata (2009) describes the experiments on language modelling which constitute Chapter 6. Finally, the application, in Chapter 7, of these semantic composition models to eye-movement prediction is also described in Mitchell et al. (2010). 


\section{Chapter 2}

\section{Background}

This chapter covers the background necessary to tackle the problem of composition in distributional models. Beginning with a general examination of the topic of semantics, we differentiate a range of philosophical attitudes to meaning and identify some of the approaches to modelling semantics which follow from these conceptions. A key contrast among these models is the difference between symbolic and non-symbolic representations. We examine this dichotomy and describe some of the vector binding mechanisms that attempt to bridge the gap between these paradigms. The discussion then returns to the topic of semantic composition, covering both what is known about its function in natural languages and also its modelling in a computational setting. Finally distributional models of semantics are dealt with in some depth and the way in which vectors combine in these models is examined.

\subsection{Theories and Models of Semantics}

Semantics is the study of meaning, and the question of what exactly a meaning is therefore forms part of its foundation. However, rather than there being a single agreed conception of what constitutes meaning, there are, in fact, a diversity of definitions and proposals. Covering all of these in depth is beyond the scope of this chapter. Instead, we will examine the main issues relevant to this thesis by describing three 
broad approaches at a high level, to uncover the main differences and constrasts in their focus.

One common conception of meaning is that of a relationship between linguistic expressions and entities and events in the world. So, for example, water refers to the physical substance with the chemical formula $\mathrm{H}_{2} \mathrm{O}$. More formally, we might define the meaning of a sentence to be its truth conditions, the conditions that must exist in the world to make it true (Davidson, 1967). Given this formulation it becomes natural to use formal logic to express the meanings of natural language expressions more clearly. For example, if $M$ and $L$ are logical symbols with the meanings $I S \_A \_M A N$ and $I S \_A \_L I A R$ respectively, then $\forall x(M x \rightarrow L x)$ expresses the meaning of the sentence All men are liars. However, employing logical expressions in this way should not be interpreted as implying that the logical expression themselves are the meanings of the natural language expressions. Instead, the two expressions share the same meaning, with the formulation in terms of logic allowing a greater precision and avoidance of amibiguity than natural language.

However, this approach ignores the fact that linguistic expressions only become meaningful through being used in context. An alternative conception views the meaning of a word as being based on the role it plays in interactions between language users. Or to put it more pithily, meaning is use. This was the attitude adopted by Wittgenstein (1953), after he rejected the view described above, that the meaning of a word is what it refers to. Within this approach, a representation of the meaning of an expression should describe the contexts in which it is used. Firth (1957) paraphrased this approach as you shall know a word by the company it keeps and this has become a standard slogan invoked by those working on distributional representations of meaning (e.g., Weeds, 2003; Lowe, 2001; Jones and Mewhort, 2007).

A third attitude is that meanings are objects in the minds of language users, with the meaning of an expression being what is understood by it (Locke, 1690). In this case, a valid representation of meaning should reflect the cognitive structures and processes 
that underlie language comprehension. This idea, that meanings are internal mental representations has an obvious appeal to cognitive scientists (see Chomsky, 2000). Within this approach, the cognitive structures that embody meanings could take many different forms, and both logical and distributional approaches have been proposed as accurate models of these mental representations (Stenning and van Lambalgen, 2008; Lowe, 2000). However, these approaches draw on fairly distinct conceptions of how cognition works. In one case, mental representations are conceived in terms of strings of discrete symbols, in the other as points in a continuous vector space. As a consequence, the contrasts between their structures and capabilities have generated substantial controversy over their relative merits as models of cognition (Fodor and Pylyshyn, 1988; Fodor and Lepore, 2002).

Moreover, a number of other data structures and algorithms have also been proposed as cognitive models of how semantic information is processed and stored. These include semantic networks (Collins and Quillian, 1969), featural models (Smith et al., 1974), associative models (Raaijmakers and Schiffrin, 1981) and cognitive architectures such as ACT-R (Anderson, 1993).

Thus, there are fundamental disagreements about what meanings are and what structure they have. However, in practice this may not present a substantial obstacle because experiments are often concerned with the relations between meanings as opposed to the nature of the meanings themselves. In other words, topics in semantics can often be investigated experimentally by simply asking subjects to make a comparison of the meanings of two expressions. For example, we can ask whether two sentences are paraphrases (Barzilay and Lee, 2003), i.e. share the same meaning, without requiring a philosophical theory of what meaning is. Similarly, we could study the extent of semantic similarity between words (Rubenstein and Goodenough, 1965), or analyse the conditions under which the meaning of one sentence is contained in or implied by the other (Dagan et al., 2006). We can even study these semantic relations without asking subjects to make deliberate conscious judgements, using a priming experimental 
paradigm to probe the semantic relations between words and phrases. Such experiments can be based on word recognition times (Simpson et al., 1989), eye-movements during reading (Pynte et al., 2008) or even event related potentials in the brain (van Berkum et al., 1999).

Not surprisingly, different types of representations are better at modelling the different types of task. For example, logical representations are more effective at modelling the deductive relations between expressions, that is whether one sentence entails another. Whereas distributional models are more appropriate for predicting similarity ratings. As a consequence, these representations are often utilised in distinct applications. For example, logic based representations have been successful in natural language database querying systems (Thompson et al., 1997), question answering (Furbach et al., 2010; Moldovan et al., 2003) and natural language interfaces for robot control (Ge and Mooney, 2009). On the other hand, distributional models have been applied to essay grading (Landauer et al., 1997), word sense discrimination (Schütze, 1998), ontology extraction (Yamada et al., 2009) and modelling semantic priming (Lund and Burgess, 1996; Landuaer and Dumais, 1997). To some extent, these applications reflect the underlying motivations of the two approaches, with logic based representations being suited to situations where modelling the relation to external entities is important and distributional representations giving better results in relation to issues of how language is used.

There are, however, other important differences in the character of these representations over and above their differing motivations and assumptions. Most importantly, logical approaches are generally based on symbolic representations whereas distributional approaches are not. In logic, individual concepts, for example an entity such as ALICE or a predicate such as FEMALE, are represented by discrete, structureless symbols, for example $a$ or $F$. On the other hand, vectors, the representational elements of distributional approaches, are continuous and have a rich internal structure. This in itself helps to explain why symbolic approaches are most effective in applications 
based on qualitative, categorical judgements, such as true vs false, whereas distributional approaches tend to be based on quantitative, continuous factors, such as similarity judgements. In addition, symbolic approaches are based on their ability to combine individual concepts to produce meaningful wholes. For example, we can combine the symbols representing ALICE and FEMALE to give $F a$, which now expresses the fact that Alice is female. In contrast, the handling of such complex structures within distributional approaches is not so well understood, and representations have tended to focus on individual, isolated words.

This problem of representing complex structures in nonsymbolic approaches has a longer history, particularly in regard to connectionism. Connectionist representations, like their distributional counterparts, are essentially vectors, and there is a substantial literature concerned with their representational capacities in comparison to the symbolic alternatives (Fodor and Pylyshyn, 1988; Smolensky, 1990; Pollack, 1990; Plate, 1991). Some recent work (Aerts and Czachor, 2004; Clark and Pulman, 2007; Widdows, 2008) has drawn on this literature in addressing the problem of semantic composition within distributional models. In Section 2.2, we will describe in more depth the differences between symbolic and non-symbolic approaches and outline some of the proposals for capturing the capabilities of symbolic representations within a nonsymbolic model.

\subsection{Symbolic and Non-symbolic Representations}

While representational systems cannot in general be unambiguously differentiated into symbolic and non-symbolic schemes, it will nonetheless be helpful to identify some key characteristics of these two approaches. In summary, symbolic representations are typically discrete, arbitrary, composable and of unbounded complexity. Whereas nonsymbolic representations are of a fixed complexity, continuous and non-arbitrary.

In a classical symbolic system, an entity, for example $A L I C E$, can be represented 
by an arbitrary symbol, say $a$. This symbol is arbitrary to the extent that any other symbol could adequately represent the same entity. All that is required is that the same symbol is always used to represent this entity.

In contrast, a nonsymbolic representation might consist of a vector of luminance values making up an image of Alice. This representation is non-arbitrary to the extent that an image of another entity, say Bob or Charlie, could not be substituted. Furthermore, these vector representations can be subject to further processing, e.g. to identify features, such as stubble or a square jaw, which might allow us to infer that Bob is more similar to Charlie than Alice.

This sort of inference, however, cannot be made in the same manner in for representations based on single symbols. If Alice, Bob and Charlie are represented by the symbols $a, b$ and $c$, there is no procedure for inferring any relation between these entities based solely on these representations. On the other hand, by introducing the symbols $M$ and $F$ representing the predicates $M A L E$ and FEMALE, we can express the commonalities between Bob and Charlie and their difference to Alice in terms of the symbolic expressions $F a, M b$ and $M c$. That is Bob and Charlie are male whereas Alice is female.

This concatenation of representations, e.g. $F$ with $a$ to give $F a$, to form compound structures with more complex meanings, e.g. Alice is female, is another defining feature of classical symbolic representations. Repeated concatenation allows strings of unbounded length to be constructed, and so symbolic models can represent structures of arbitrary complexity. Significantly, concatenation is reversible, so that complex structures can be broken down into the original constituents. This allows symbolic processes to be defined in terms of breaking down complex structures and recombining the parts. Thus, while the atomic symbols, due to their arbitrary nature, are vacuous in themselves, complex representations formed by concatenating these symbols can be processed meaningfully. The prototypical example of such processing would be deductive inference, which would allow us to infer $L b$, Bob is a liar, from 
$M b \wedge \forall x(M x \rightarrow L x)$, Bob is a man and all men are liars. In fact, this inference to $L b$ would be valid whatever the meaning of the symbols $M, L$ and $b$, as what matters is that the syntactic form of the inference is valid. This property makes deductive inference particularly amenable to symbolic processing. Since, by simply manipulating symbols we can derive deductively valid inferences, without any reference to what the symbols stand for.

Inductive inference, however, usually cannot be formulated in such an abstract manner, and is more commonly implemented in terms of non-symbolic representations. A typical problem of inductive inference would be the discovery of general properties from specific instances. So, for example, given a series of specific images of men and women, we might try to infer a general procedure which would allow us to categorise new images by gender. Various approaches to solving such a problem exist, including back-propagation networks (Rumelhart et al., 1986) and support vector machines (Vapnik, 1995). A common property of these inductive algorithms is that the predicted class of a novel instance depends most strongly on the classes of the nearest training examples. In effect, the learning process involves finding those respects in which similarity is most predictive of the desired classification. Thus, flexibility in determining the similarity of representations is frequently a useful property of nonsymbolic representations for inductive tasks. Vector similarities, for example, lie on a continuous range of values and can be parameterised in a variety of ways. In contrast, two atomic symbols are simply either the same or different.

On the other hand, sophisticated measures of similarity have been investigated for more complex symbolic structures, such as methods based on structural alignment (Falkenhainer et al., 1989) or representation distortion (Hahn et al., 2003). These algorithms typically involve finding a mapping from the sub-parts of one structure into those of the other, and thus are well suited to representations which are constructed by combining a number of parts into a whole. Non-symbolic representations typically do not have such constituent structure. 
Moreover, whereas symbolic structures can be of unbounded size or complexity, non-symbolic representations are typically fixed in structure, for example vectors of a given dimension. In particular, the inductive learning processes referred to above usually require all representations to be of a limited, fixed size, and will often break down when applied to structures of too high a complexity.

Such differences between symbolic and non-symbolic approaches led Fodor and Pylyshyn (1988) to propose that cognition is fundamentally symbolic and to criticise the then increasingly popular connectionist models. Connectionist models, they argued, would be unable to represent structures such as Alice trusts Bob adequately. A symbolic representation, $a T b$, both distinguishes the roles of Alice and Bob, by being distinct from $b T a$, and also maintains the identity of Alice and Bob, by allowing the representation to be decomposed to recover the symbols $a$ and $b$. Connectionist representations, they argued, would either fail to differentiate the roles of trusting and being trusted, or would fail to identify the same individual in distinct roles, for example Bob in Alice trusts Bob and Bob lies.

In response, many connectionist researchers began looking for methods to overcome these criticisms. Their proposals are most clearly understood as attempts to harness the power of symbolic processing within a connectionist framework. Specifically, they sought to find some means of concatenating representations in a way that would allow representations with complex part-whole structures.

Smolensky (1990), for example, proposed the use of tensor products as a means of binding one vector to another to produce structured representations. The tensor product $\mathbf{u} \otimes \mathbf{v}$ is a matrix whose components are all the possible products $u_{i} v_{j}$ of the components of vectors $\mathbf{u}$ and $\mathbf{v}$. Figure 2.1 illustrates the tensor product for two threedimensional vectors $\left(u_{1}, u_{2}, u_{3}\right) \otimes\left(v_{1}, v_{2}, v_{3}\right)$. As a mechanism for binding vectors, it is essentially a connectionist version of concatenation, in that it allows two representations to be bound and also allows a bound representation to be broken down into the constituents from which it was formed. However, in this approach, the representa- 


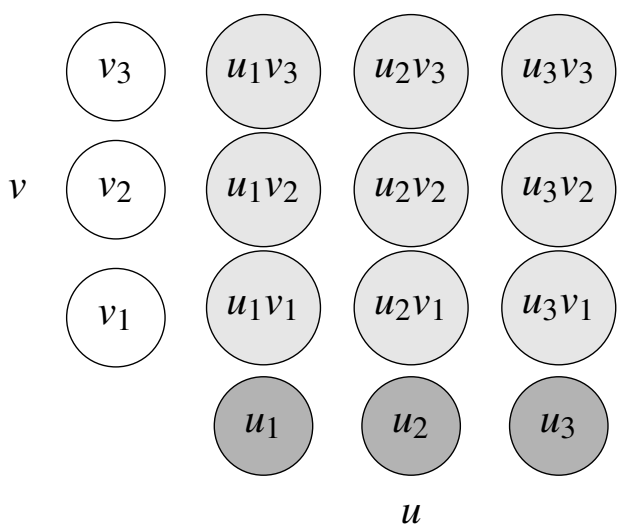

Figure 2.1: The tensor product of two three-dimensional vectors $u$ and $v$.

tions of complex structures suffer from the curse of dimensionality, with the number of dimensions increasing exponentially with the number of bindings.

Hinton (1990) makes clear that one of the strengths of symbolic representations is the handling of structures of unbounded complexity, and discusses how this might be implemented in terms of fixed dimensionality connectionist representations. After examining the representation of structures containing multiple component parts, he suggests that both full and reduced descriptions are required to handle them. Full descriptions provide the details of the constituents in a high dimensional representation, while the reduced description captures the essential properties of the combined structure using fewer dimensions, and also allows the full description to be recovered when required. Essentially, this requires a method for reversibly binding two vectors into a single vector which has the same dimensionality as its components.

Holographic reduced representations (HRR, Plate, 1991) are one implementation of this idea where the tensor product is projected onto the space of the original vectors, thus avoiding any dimensionality increase. The projection is defined in terms of circular convolution a mathematical function that compresses the tensor product of two vectors. The compression is achieved by summing along the transdiagonal elements of the tensor product. Noisy versions of the original vectors can be recovered by means of 
circular correlation which is the approximate inverse of circular convolution. The success of circular correlation crucially depends on the components of the $n$-dimensional vectors $\mathbf{u}$ and $\mathbf{v}$ being real numbers and randomly distributed with mean 0 and variance $\frac{1}{n}$.

Whereas holographic reduced representations bind vectors using a fixed, predetermined projection function (circular convolution), the Recursive Auto-Associative Memory (RAAM) proposed by Pollack (1990) learns how to bind representations using an auto-associative feedforward network. To bind pairs of $n$ dimensional vectors, this network would consist of a $2 n$ dimensional input layer, an $n$ dimensional hidden layer and a $2 n$ dimensional output layer. To learn a binding function, the inputs and outputs of such a network are presented with identical training data, consisting of the pairs of vectors to be bound. The network therefore learns how to project the vector pair seen in the input layer down onto a single $n$ dimensional vector in such a way that it can then reconstruct the original vector pair on the output layer. Crucially, recursiveness is introduced to this auto-associative structure by allowing for some of the training vectors to be representations constructed by the hidden layer of the network. In other words, vector pairs presented at the inputs of the auto-associative network are projected, in the hidden layer, onto a single vector representation, which may itself then be used as an input to the network and bound with other representations. This allows hierarchical structures to be represented and processed, for example in learning grammatical structures (Pollack, 1990).

Another major difference between the proposals of Smolensky (1990) and Plate (1991) in comparison to that of Pollack (1990) is that whereas the former are based on the tensor product of two vectors the latter is based on their cartesian product. If we have two $n$ dimensional vectors, $\mathbf{u}$ and $\mathbf{v}$, then their cartesian product is the $2 n$ dimensional vector whose first $n$ components are the components of $\mathbf{u}$ and whose remaining components are those of $\mathbf{v}$. In terms of the structure of the RAAM network, this means that the bound representations formed on the hidden layer are based on a 
sum of each vector multiplied by a matrix ${ }^{1}$ : i.e., $A \mathbf{u}+B \mathbf{v}$. In other words, the bound vector is based on additive combinations of the components of the constituent vectors. In contrast, bindings based on the tensor product use multiplicative combinations of the constituent vectors.

What these proposals all have in common is that the binding functions are all designed to mimic the characteristics of symbol concatenation. The concatenation of two symbols creates a new representation which can then be broken down to recover the original symbols. Analogously, these vector binding operations are designed to allow a pair of vectors to be bound into a single representation from which the original constituents can be recovered at some later juncture. From this perspective they can be seen as connectionist models of memory for complex structures. In fact, this is explicit in the work of Plate (1991) who refers to his architecture as a convolution memory and also in the name recursive auto-associative memory chosen by Pollack (1990).

The memory function of these proposals can be seen in the tasks they have been applied to. For example, Pollack (1990) applies the RAAM architecture to the storage and recall of letter sequences. Circular convolution has been applied to memorising pen trajectories for handwritten digits (Plate, 1993) and complex semantic structures, consisting of agents playing particular roles in various actions (Plate, 1995).

This latter application suggests that such binding operations may provide the necessary framework for composing distributional vectors to form representations of complex semantic constructions. Recent work (Aerts and Czachor, 2004; Clark and Pulman, 2007; Widdows, 2008), has examined this possibility in more depth, and in Chapter 5 we will evaluate the tensor product and circular convolution as models of semantic composition on their ability to predict similarity judgements for short phrases.

Before that evaluation, however, Chapter 4 will place those binding operations in the context of a more general framework for understanding semantic composition, alongside a number of other proposals. To motivate that framework, and the other

\footnotetext{
${ }^{1}$ In addition, the hidden layer nodes apply a non-linear sigmoid activation function to the components of the resulting vector.
} 
proposals it contains, Section 2.3 will discuss the general nature of semantic composition, identifying characteristics and issues which may be relevant to understanding the problem and formulating an approach.

\subsection{Semantic Composition}

Compositionality allows languages to construct complex meanings from combinations of simpler elements. This property is often captured in the following principle: the meaning of a whole is a function of the meaning of the parts (Partee, 1995, p. 313). Therefore, whatever approach we take to modeling semantics, representing the meanings of complex structures will involve modeling the way in which meanings combine. Let us express the composition of two constituents, $\mathbf{u}$ and $\mathbf{v}$, in terms of a function acting on those constituents:

$$
\mathbf{p}=f(\mathbf{u}, \mathbf{v})
$$

The vector $\mathbf{p}$ here represents the single meaning which results from combining the meanings represented by $\mathbf{u}$ and $\mathbf{v}$. This operation of combining multiple constituent parts into a single whole is the key characteristic of composition and sets it apart from the problem of, say, modelling how the meaning of individual words are modified or selected in context (Erk and Pado, 2008). This latter task, rather than producing a single combined representation, produces one modified representation for each constituent. While modelling the effects of context on the semantics of individual words is a closely related task, it does not by itself provide a model of composition.

Partee (1995, p. 313) suggests a further refinement of the above principle taking the role of syntax into account: the meaning of a whole is a function of the meaning of the parts and of the way they are syntactically combined. We thus modify the composition function in (2.1) to account for the fact that there is a syntactic relation $R$ between constituents $\mathbf{u}$ and $\mathbf{v}$ :

$$
\mathbf{p}=f(\mathbf{u}, \mathbf{v}, R)
$$


Unfortunately, even this formulation may not be fully adequate. Lakoff (1977, p. 239), for example, suggests that the meaning of the whole is greater than the meaning of the parts. The implication here is that language users are bringing more to the problem of constructing complex meanings than simply the meaning of the parts and their syntactic relations. This additional information includes both knowledge about the language itself and also knowledge about the real world. Thus, full understanding of the compositional process involves an account of how novel interpretations are integrated with existing knowledge. Again, the composition function needs to be augmented to include an additional argument, $K$, representing any knowledge utilized by the compositional process:

$$
\mathbf{p}=f(\mathbf{u}, \mathbf{v}, R, K)
$$

The difficulty of defining compositionality is highlighted by Frege $(1884$, p. x) himself who cautions never to ask for the meaning of a word in isolation but only in the context of a statement. In other words, it seems that the meaning of the whole is constructed from its parts, and the meaning of the parts is derived from the whole. Moreover, compositionality is a matter of degree rather than a binary notion. Linguistic structures range from fully compositional (e.g., black hair), to partly compositional syntactically fixed expressions, (e.g., take advantage), in which the constituents can still be assigned separate meanings, and non-compositional idioms (e.g., kick the bucket) or multi-word expressions (e.g., by and large), whose meaning cannot be distributed across their constituents (Nunberg et al., 1994).

Despite the foundational nature of compositionality to language, there are significant obstacles to understanding what exactly it is and how it operates. Most significantly, there is the fundamental difficulty of specifying what sort of "function of the meanings of the parts" is involved in semantic composition (Partee, 2004, p. 153). Fodor and Pylyshyn (1988) attempt to characterize this function by appealing to the notion of systematicity. They argue that the ability to understand some sentences is intrinsically connected to the ability to understand certain others. For example, no- 
one who understands Alice sues Bob fails to understand Bob sues Alice. Therefore, the semantic content of a sentence is systematically related to the content of its constituents and the ability to recombine these according to a set of rules. In other words, if one understands some sentence and the rules that govern its construction, one can understand a different sentence made up of the same elements according to the same set of rules. In a related proposal, Holyoak and Hummel (2000) claim that in combining parts to form a whole, the parts remain independent and maintain their identities. This entails that Alice has the same independent meaning in both Alice sues Bob and Charlie represents Alice.

Aside from the difficulties of determining what systematicity means in practice (Pullum and Scholz, 2007; Spenader and Blutner, 2007; Doumas and Hummel, 2005), it is worth noting that semantic transparency, the idea that words have meanings which remain unaffected by their context, contradicts Frege's (1884) claim that words only have definite meanings in context. Consider for example the adjective good whose meaning is modified by the context in which it occurs. The sentences Charlie is a good neighbor and Charlie is a solicitor do not imply Charlie is a good solicitor. In fact, we might expect that some of the attributes of a good lawyer are incompatible with being a good neighbor, such as nit-picking over details, or not giving an inch unless required by law. More generally, the claims of Fodor and Pylyshyn (1988) and Holyoak and Hummel (2000) arise from a preconception of cognition as being essentially symbolic in character. While it is true that the concatenation of any two symbols (e.g., $G$ and $S$ ), will compose into an expression (e.g., $G S$ ), within which both symbols maintain their identities, we cannot always assume that the meaning of a phrase is derived by simply concatenating the meaning of its constituents. Although the phrase good solicitor is constructed by concatenating the symbols good and solicitor, the meaning of good will vary depending on the nouns it modifies.

Interestingly, Pinker (1994, p. 84) discusses the types of functions that are not involved in semantic composition while comparing languages, which he describes as 
discrete combinatorial systems, against blending systems such as colour mixing. He argues that languages construct an unlimited number of completely distinct combinations with an infinite range of properties. This is made possible by creating novel, complex meanings which go beyond those of the individual elements. In contrast, for a blending system the properties of the combination lie between the properties of its elements, which are lost in the average or mixture. To give a concrete example, a brown cow does not identify a concept intermediate between brown and cow (Kako, 1999, p. 2). Thus, composition based on averaging or blending would produce greater generality rather than greater specificity.

Experiments on sentence recall (Sachs, 1967, 1988; Begg, 1971) also give us some indication of what semantic composition is not. These show that memory for the meaning of a sentence and memory for its surface realisation behave in quite independent ways, with subjects typically remembering a sentence's meaning for much longer than they can recall its specific wording. Thus, whatever a meaning is, it is clear that is not simply a memory of the superficial sequence of words used to express it. In other words, a mechanism for binding representations to form a single memory trace, from which the original constituents can be accurately recalled, is not the the same thing as a model for the composition of those representations. To a great extent this is because the same meaning can be constructed from quite disparate constituents: e.g. thespian mother and actress parent. If language users derive the same meaning for these phrases, and are subsequently unable to recall which phrase was used to express that meaning, then a valid model of semantic composition should also behave in this way. In particular, the model should compose semantic representations for these phrases to produce the same result in both cases, in effect erasing the details of the original constituents.

The most common approach to modelling semantic composition has been to use a combination of symbolic logic and the lambda calculus (Montague, 1974; Blackburn and Bos, 2005). In this framework, each syntactic type corresponds to a specific functional type, with composition consisting of the application of the function representing 
one constituent to arguments representing the other constituents. So, for example, the proper noun Bob could be represented by the logical symbol $b$ denoting a specific entity, whereas a verb like lies, might be represented by a function from entities to propositions, expressed in lambda calculus as $\lambda x . L x$. Applying this function to the entity $b$ yields the logical formula $L b$ as a representation of the sentence Bob lies. It is worth noting that the entity and predicate within this formula are represented symbolically, and that the connection between a symbol and its meaning is an arbitrary matter of convention.

On one hand, this symbolic character of logical representations is advantageous as it allows processing to be carried out syntactically. The laws of deductive logic in particular can be defined as syntactic processes which act irrespective of the meanings of the symbols involved. On the other hand, abstracting away from the actual meanings may not be fully adequate for modeling semantic composition. For example, while intersective adjectives can be handled in terms of predicate conjunction, e.g., Charlie is a male solicitor corresponds to $M c \wedge S c$, this approach cannot handle the context sensitive adjectives discussed above. Charlie is a good solicitor is not equivalent to the conjunction of Charlie is good and Charlie is a solicitor. In this case, the contribution of good depends on the noun it modifies, and this context dependence cannot be adequately represented by simple conjunction.

These issues suggest that associating the meaning of a word with a single discrete symbol may be inadequate. Modelling the complex interactions of meaning in the process of composition is likely to require representations with more sophisticated internal structure, and intuitively both good and solicitor seem to contain more content than can be adequately captured in terms of representation by a single predicate. Perhaps such complex concepts can be broken down into simpler elements and the process of composition defined in terms of the interaction of these components. However, this in turn raises the problems of how to identify the atomic elements and how to build the complex concepts associated with whole words out of these constituents. 
Instead, this thesis adresses the problem of composition within distributional models of semantics. These representations have a rich internal structure, being based on vectors, and can be derived from the empirical patterns of word usage collected from a suitable corpus.

\subsection{Distributional Semantics}

Semantic space models are based on two assumptions: (1) words with similar meanings are found in similar contexts, and (2) semantic similarity can be modelled in terms of the spatial similarity of vector representations. Together, these assumptions motivate models in which vector based representations of semantics are constructed from the distribution of words across contexts, such that words with similar meanings are found close to each other in the space. Putting this into practice means deriving vectors from the distributional properties of words, and then applying some metric to those vectors to calculate semantic similarities. These semantic similarities can then be used in variety of tasks, including modelling semantic priming (Landuaer and Dumais, 1997; Lund and Burgess, 1996) and human similarity judgments (McDonald, 2000), automatic thesaurus extraction (Grefenstette, 1994) and word sense discrimination (Schütze, 1998) and disambiguation (McCarthy et al., 2004)

The underlying motivations and assumptions of these models have their origins in a variety of disparate sources. For example, the idea of representing word meaning in a geometrical space can be traced back to Osgood et al. (1957), who used elicited similarity judgments to construct semantic spaces. Subjects rated concepts on a series of scales whose endpoints represented polar opposites (e.g., happy-sad); these ratings were further processed with factor analysis, a dimensionality reduction technique, to uncover latent semantic structure. In this study, meaning representations were derived from psychological data, thereby allowing the analysis of differences across subjects. Unfortunately, multiple subject ratings are required to create a representation for each 
word, which in practice limits the semantic space to a small number of words. Similar ideas are also employed by the vector space model in information retrieval (Salton et al., 1975; Deerwester et al., 1990) as a practical solution to the engineering problem of how to match documents to queries. In this approach, both documents and queries are represented as vectors, and the match between them is based on their spatial similarity. However, instead of using subject ratings to construct these vectors, they are based on word counts from a corpus.

The origin of the other crucial ingredient of semantic space models, the idea that the semantic properties of words can be inferred from their distributional properties, is commonly associated with the work of Firth (1957) and Harris (1954). Firth (1957) proposed the dictum you shall know a word by the company it keeps in response to the usage based theory of meaning described by Wittgenstein (1953). This has now become a standard slogan invoked by those working on distributional approaches to justify the representation of a word's meaning in terms of the contexts it occurs in. However, this idea also has strong connections to structural linguistics, which makes widespread use of distributional analyses in syntax and phonology, for example. Harris (1954) is generally credited with the hypothesis that similar forms of analysis could be applied to the semantic properties of words.

Perhaps because the semantic space approach lacks a single well-defined theoretical basis, but instead derives from multiple overlapping influences, the practical implementations are themselves diverse and varied. In particular, three main choices need to be made to turn these ideas into a concrete model of semantics. First, the concept of context needs to be given a practical definition. A word's context could be as wide as the whole document it occurs in, or as narrow as a word immediately beside it. Also relevant is the question of how we handle the syntactic structure of the context, or whether that structure is ignored altogether, opting for a bag-of-words treatment. Second, given a matrix of the occurrences of words across contexts, we need to choose some method of constructing word vectors from that data. A simple choice would be 
to associate each context one-to-one with a component of the vector, with the value of that component being some function of the frequency count of the corresponding context. Alternatively, we might apply some dimensionality reduction procedure to these raw vectors to uncover the latent semantic factors which underlie the raw frequencies. Third, some metric for comparing vectors within the derived space needs to be chosen, to allow the calculation of similarities.

As an example of such a space, we will outline one of the models described in the survey of Bullinaria and Levy (2007), which performs relatively well across a range of tasks. Context, in the case of this model, is defined in terms of word co-occurrence within a short distance. So, given a word for which we wish to build a distributional representation (the target word) we identify tokens of that word in the corpus, define a short window, say five words, either side of the target word tokens and then compile counts of words which occur in those windows (the context words). Typically, our set of context words will not include function words, which are not particularly semantically informative, and will also exclude infrequent word types, to avoid noise due to sparseness. Thus, the context counts for our target word are based on co-occurrences with a set of the most common, say top 2,000, content words. The effects of sparseness may also be reduced by removing semantically irrelevant inflectional structure from the word tokens, in other words by stemming or lemmatising.

Each context word then defines a component of the semantic vector representing the target word. So, if we have 2,000 context words, our vectors have 2,000 components, with the value of each component being based on the co-occurrence count for the corresponding context word. These raw frequencies could be used directly as the vector components, but it is common to transform the counts first. In particular, the raw counts emphasise the contribution of high frequency words, diminishing the influence of low frequency but semantically informative context words. This can be countered by using a ratio of probabilities measure instead of the raw frequencies. If $p\left(c_{i} \mid t\right)$ is the conditional probability of a context word $c_{i}$ given the target word $t$, and $p\left(c_{i}\right)$ is the 
overall probability of context word $c_{i}$, then we can define the components, $v_{i}$, of the vector, $\mathbf{v}$, representing $t$ in terms of the ratio of these probabilities:

$$
v_{i}=\frac{p\left(c_{i} \mid t\right)}{p\left(c_{i}\right)}
$$

These values now scale the context counts such that all the components are distributed around one, and gives equal weight to both high and low frequency context words.

Having constructed vectors in this way, we require some method of calculating similarities and the cosine measure is commonly employed to this end.

$$
\cos (\mathbf{u}, \mathbf{v})=\frac{\mathbf{u} \cdot \mathbf{v}}{\sqrt{\mathbf{u} \cdot \mathbf{u}} \sqrt{\mathbf{v} \cdot \mathbf{v}}}
$$

This essentially measures the cosine of the angle between the vectors $\mathbf{u}$ and $\mathbf{v}$, thus ignoring the length of the vectors involved. In contrast, for the Euclidean distance, length does have an effect. Alternatively, instead of using a geometric metric, a statistical or information theoretic measure could be employed, such as the Kullback-Leibler divergence of the conditional probability distributions over contexts for the two target words.

This then defines a simple semantic space which can be used as a model of semantic similarity between words. There are, however, many other ways of implementing such a model. For example, the Hyperspace Analogue to Language (HAL, Lund and Burgess, 1996) model also uses a window based approach to defining co-occurrence, but in this case the counts are weighted by the distance between the target word and context word. In addition, separate counts are maintained for occurrences to the left and right of the target word. So that if counts are gathered for $n$ context words, this results in a semantic vector with $2 n$ components. To select this set of context words, the components with the highest variance are chosen, rather than the most frequent, and the vector components are then based on raw counts for these words. Similarity is measured in terms of Euclidean distance between vectors, having normalised the lengths of all vectors. 
Latent Semantic Analysis (LSA, Landuaer and Dumais, 1997) is another implementation of the same ideas, using a fairly different approach. Here, context is defined in terms of documents, for example entries in an encyclopedia, with each document defining a separate context. Counts for the occurrence of each target word in each document are collated and entered into a word-document matrix, and these raw values are transformed to smooth the distribution of values and weight each word by its context specificity. Singular Value Decomposition (Golub et al., 1981), a dimensionality reduction technique, is then applied to this matrix, producing lower dimensional vectors representing words and documents. Essentially, SVD identifies the main components of variation in the original data and constructs an approximation based on retaining those components. In the context of LSA, this can be thought of as identifying a set of latent semantic factors which account for the differences in vocabulary of the documents. Semantic similarity is then measured in terms of the cosine measure on the reduced word vectors.

Both LSA and HAL have been used to model semantic priming (Landuaer and Dumais, 1997; Lund and Burgess, 1996). This is an effect where the recognition of a given word is facilitated when it is preceded by semantically similar or associated words. Thus, as cognitive models of semantic representations, these models appear to be effective predictors of how such information is retrieved and processed. Furthermore, HAL has been applied to modelling the cerebral asymmetries in word recognition (Burgess and Lund, 1998) and also lexical emotional connotations (Burgess and Lund, 1997). The applications of LSA have included essay grading (Landauer et al., 1997) and enhancing $n$-gram language models with long range semantic information (Bellegarda, 2000; Coccaro and Jurafsky, 1998).

This last application to language modelling is notably appropriate, in that it is reasonable to expect a model of meaning based on the distribution of words across contexts to be particularly useful in exploiting semantic factors to predict which words are likely to occur in a given context. However, the cosine similarities produced by LSA 
are unfortunately not practically conducive to deriving probabilities, and these values typically have to be transformed in some ad-hoc manner to derive an effective language model. Moreover, the whole statistical structure of the LSA approach is somewhat lacking in rigour. Rather than being an actual probabilistic model of the distribution of words across contexts, it is instead an ad-hoc set of procedures for constructing vector representations.

The probabilistic LSA model (pLSA, Hofmann, 2001) addresses these criticisms, and derives a generative model that accounts for the distribution of words across the documents of a corpus. Key to this model are latent topics, which are essentially unigram distributions over words. Each document is then represented as a particular mixture of topics, which determines its characteristic vocabulary. Latent Dirichlet Allocation (LDA, Blei et al., 2003) extends this model by introducing a set of Dirichlet priors $^{2}$ which determine how document topic mixtures are generated. This means the LDA model is a generative model for entire corpora, with documents treated as bagsof-words.

For each document, $d$, in the corpus we draw the mixing proportion over topics $\theta_{d}$ from a Dirichlet prior with parameters $\alpha$. Next, for each of the $N_{d}$ words $w_{d n}$ in document $d$, a topic $z_{d n}$ is drawn from the topic distribution defined by $\theta_{d}$. Finally, a word token $w_{d n}$ is drawn from a unigram distribution conditioned on the chosen topic, $p\left(w_{d n} \mid z_{d n}\right)$. These word probabilities are parametrised by a matrix, $\beta_{i j}=p(w=i \mid z=j)$. This model then defines the probability of an $M$ document corpus $D$ given the parameters $\alpha$ and $\beta$.

$$
P(D \mid \alpha, \beta)=\prod_{d=1}^{M} \int P\left(\theta_{d} \mid \alpha\right)\left(\prod_{n=1}^{N_{d}} \sum_{z_{d n}} P\left(z_{d n} \mid \theta_{d}\right) P\left(w_{d n} \mid z_{d n}, \beta\right)\right) d \theta_{d}
$$

Training the LDA model involves maximising the $\log$ likelihood of the corpus $D$ by

\footnotetext{
${ }^{2}$ The Dirichlet distribution is a commonly used prior for multinomials $P(\theta)=\frac{1}{B\left(a_{1} \ldots, a_{n}\right) \prod_{i=1}^{n} \theta_{i}^{a_{i}-1}}$ where $a_{1} \ldots, a_{n}$ are the parameters of the prior and the normalizing constant $B\left(a_{1} \ldots, a_{n}\right)$ is the $n$-dimensional Beta function. One important reason for the use of the Dirichlet prior in the case of multinomial parameters is its mathematical expedience. It is a conjugate prior for the multinomial distribution. This means that the prior and the likelihood can easily combine according to Bayes' law to specify the posterior distribution.
} 
setting the parameters $\alpha$ and $\beta$, which Blei et al. (2003) optimise using a variational form of the expectation maximization algorithm. Alternatively, a slightly modified form of the model can be optimised using Gibbs sampling (Griffiths et al., 2007). Either way, the representation of words in this approach are based on their probabilistic dependence on the latent topic variables.

Blei et al. (2003) evaluate LDA as a language model, in terms of its ability to account for the unigram vocabularies of the documents within a corpus, rather than investigating its value in creating semantic representations. Griffiths et al. (2007), in contrast, evaluate the topic based representations on semantic tasks, such as predicting word association norms and identifying synonyms, and find that LDA outperforms LSA. However, the LDA framework does not unambiguously identify the appropriate metric for calculating semantic similarity, and some ad-hoc manipulation can be required to fit semantic tasks to its probabilistic approach.

The preceding discussion has considered distributional models in which the internal structures of contexts are ignored and processed in a bag-of-words manner. One alternative approach is to incorporate the syntactic structure of contexts into the procedure for constructing vectors (Grefenstette, 1994; Lin, 1998; Padó and Lapata, 2007). For example, the simple context word by target word co-occurrence counts can be broken down further to take account of the syntactic dependency between them. This results in sparser counts, but which may be more semantically informative.

Another option is to use the sequential structure of context words. To some extent HAL (Lund and Burgess, 1996) already explots this structure, albeit simplistically, with its weighting by distance between context and target words. Jones and Mewhort (2007) propose a model that makes use of the linear order of words in a context in a more sophisticated manner. Their model represents words by high-dimensional holographic vectors. Each word is is assigned a random ${ }^{3}$ environmental vector. Contextual information is stored in a lexical vector which is computed with the aid of the en-

\footnotetext{
${ }^{3}$ Vector components are sampled at random from a Gaussian distribution with $\mu=0$ and $\sigma=\frac{1}{\sqrt{D}}$ where $D=2,048$.
} 
vironmental vectors. Specifically, a word's lexical vector is the superposition of the environmental vectors corresponding to its co-occurring words in a sentence. Order information is the sum of all $n$-grams that include the target word. The $n$-grams are encoded with the aid of a place-holder environmental vector $\Phi$ and circular convolution (Plate, 1995). The order vector is finally added to the lexical vector in order to jointly represent structural and contextual information. Despite the fact that these vectors contain information about multi-word structures in the contexts of target words, they are, nonetheless, still fundamentally representations of individual isolated target words. Circular convolution is only used to bind environmental vectors, which being random contain no semantic information. To make a useful semantic representation of a target word, the vectors representing its contexts are summed over, producing a vector which is no longer random and for which circular convolution is no longer optimal. Sahlgren et al. (2008) provide and alternative to convolution by showing that order information can also be captured by permuting the vector coordinates.

So far the discussion has centered on the creation of semantic representations for individual words. The focus of this thesis is the composition of vector-based semantic representations to create representations for phrases and sentences, which has thus far received relatively little attention. However, an alternative is not to compose at all but rather create semantic representations for phrases in addition to words. If a phrase is frequent enough, then it can be treated as a single target unit, and its occurrence across a range of contexts can be constructed in the same manner as described above. Baldwin et al. (2003) apply this method to model the decomposability of multi-word expressions such as noun compounds and phrasal verbs. Taking a similar approach, Bannard et al. (2003) develop a vector space model for representing the meaning of verb-particle constructions. In the limit, such an approach is unlikely to work as semantic representations for constructions that go beyond two-words will be extremely sparse. 


\subsubsection{Composition in Distributional Models}

Vector addition or averaging (which are equivalent under the cosine measure) is the most common form of vector combination (Landuaer and Dumais, 1997; Foltz et al., 1998). However, vector addition is not a suitable model of composition for at least two reasons. Firstly, it is insensitive to syntax and word order. Because vector addition is commutative, it assigns the same representation to any sentence containing the same constituents irrespective of their syntactic relations. It is therefore a bag-of-words model of composition. In contrast, there is ample empirical evidence that syntactic relations across and within sentences are crucial for sentence and discourse processing (Neville et al., 1991; West and Stanovich, 1986). Secondly, addition simply blends together the content of all words involved to produce something in between them all. Ideally, we would like a model of semantic composition that generates novel meanings by selecting and modifying particular aspects of the constituents participating in the composition. Kintsch (2001) attempts to achieve this in his predication algorithm by modeling how the meaning of a predicate (e.g., run) varies depending on the arguments it operates upon (e.g, the horse ran vs. the color ran). The idea is to add not only the vectors representing the predicate and its argument but also the neighbors associated with both of them. The neighbors, Kintsch argues, can strengthen features of the predicate that are appropriate for the argument of the predication.

Tensor products have been recently proposed as an alternative to vector addition. (Aerts and Czachor, 2004; Clark and Pulman, 2007; Widdows, 2008). However, as illustrated in Figure 2.1, these representations grow exponentially as more vectors are combined. This fact undermines not only their tractability in an artificial computational setting but also their plausibility as models of human concept combination. Interestingly, Clark et al. (2008) try to construct a tensor product based model of vector composition which makes an explicit connection to models of linguistic composition. In particular, they show how vector-based semantics can be unified with a compositional theory of grammatical types. Central to their approach is the association of each 
grammatical type with a particular rank of tensor. So, for example, if we take nouns as being associated with simple vectors, then an adjective as a noun modifier would be associated with a matrix, i.e. a vector transformation. Clark et al. (2008) do not suggest concrete methods for constructing or estimating the various tensors involved in their model. Instead, they are more interested in its formal properties and do not report any empirical tests of this approach.

A number of other proposals have also focused on the alleged theoretical benefits of representational formalisms that go beyond simple vectors. In the approach of Rudolph and Giesbrecht (2010), semantic representations are made in terms of matrices, with semantic composition being based on matrix multiplication. Clarke et al. (2010) propose instead the use of quotient algebras. In neither case are there clear empirical evaluations of these schemes.

Unfortunately, comparisons across vector composition models have been few and far between. The merits of different approaches are illustrated with special purpose examples and large scale evaluations are uniformly absent. For instance, Kintsch (2001) demonstrates how his own composition algorithm works intuitively on a few hand selected examples but does not provide a comprehensive test set (see Frank et al. 2007 for a criticism of Kintsch's 2001 evaluation standards). In a similar vein, Widdows (2008) explores the potential of vector product operations for modeling compositional phenomena in natural language, again on a small number of hand picked examples.

There is thus considerable scope for empirical evaluations of semantic composition in distributional models. Ideally such evaluations should be based on substantial quantities of natural data and demonstrate both the practical application and cognitive relevance of the compositional models. In this thesis, we achieve this by applying distributional models of composition to three tasks. We first quantify the ability of our composition models to predict subject similarity ratings in Chapter 5 by correlating their computed similarities against a large dataset of judgements gathered for short phrases and sentences from native English speakers. We then evaluate the ability of 
these models to predict long range semantic dependencies in natural text in Chapter 6 by employing them in the practical task of language modelling. Finally, in Chapter 7 we assess the relevance of the semantic relations identified by our models to cognitive processes of comprehension in a regression analysis of reading times from an eye-movement study.

\subsection{Conclusions}

In this chapter we presented an overview of concepts and issues relevant to the understanding of composition in distributional representations. In particular, we described the assumptions and implementations of various distributional models of semantics, which will inform the construction of our underlying semantic representations in Chapter 3. Our discussion of the general nature of semantic composition serves as a background against which to motivate the framework we propose for vector composition in Chapter 4 and helps to elucidate the results of our experiments. Finally, the discussion of symbolic and non-symbolic representations serves to contextualise the various approaches to modelling semantic composition, such as those based on formal logic or vector binding. With regard to this last proposal, it is worth noting here that these binding functions are designed to mimic the syntactic operation of symbol concatenation as opposed to the semantic operation of composition. We will return to this distinction in the discussion of their empirical evaluation.

An important unresolved question concerns the precise nature of the function of the parts involved in semantic composition. An answer to this question would narrow the space of hypotheses to be tested and help to focus our empirical investigations. Instead, lacking a precise answer, we will describe a fairly broad range of existing proposals for vector composition functions in Chapter 4 and propose a few novel functions based on simplicity and intuition. We have, however, discussed two possibilities for what semantic composition is not. Pinker (1994) claims that whatever it is, it is not 
blending or averaging and the experiments of Sachs (1967) suggest that it is not simply memorisation of the constituents and the structure in which they occurred. Again, we will return to these hypotheses in discussing our empirical results.

To obtain these empirical results, and so investigate the issues raised in this chapter, we first need to construct the basic distributional representations on which our composition functions will operate. In Chapter 3, we evaluate a number of such models and select those that we wish to use in the experimental evaluations of Chapters 5, 6 and 7. 


\section{Chapter 3}

\section{Constructing Distributional}

\section{Representations of Word Meaning}

This chapter will describe the distributional models we constructed, and their evaluation as representations of individual words. These models will then form the basis of our later experiments in representing larger structures, such as phrases and sentences. We implemented a number of different approaches, experimenting with a range of parameter settings, and evaluated the computed word similarities of the resulting models on two tasks. The first of these involved predicting similarity judgements for the word pairs gathered in the WordSim353 database (Finkelstein et al., 2002). The second task was based on a TOEFL task, commonly used to evaluate distributional models (Landauer and Dumais, 1996; Bullinaria and Levy, 2007; Padó and Lapata, 2007), and involved identifying synonyms from amongst a set of alternatives.

\subsection{Aims}

In choosing the representations on which to investigate models of composition we had a number of aims. First, we wanted to ensure that we were working with models which represented the semantics of individual words effectively. Representations which show 
poor evaluations at the word level are unlikely to be give reliable results at the phrase level. Second, we wanted to select representations which were robust and could be processed efficiently. Ideally, our models ought to show consistent performance across data and tasks, while also avoiding approaches which consume excessive computing time or are badly affected by problems of sparsity on infrequent words. These latter issues are particularly relevant to representations of high dimension.

Although we based the selection of distributional representations on word level evaluations, this choice nonetheless has consequences for our compositional models and the phrase level evaluations. The effectiveness of any operation which combines distributional representations will almost inevitably be dependent on the particular structure of those constituent representations. Therefore, rather than select a single model with which to proceed, we selected two models based on distinct approaches. In later chapters, this will allow us to examine the dependence between the effectiveness of compositional models and the form of the underlying word level representation.

Of the selected models, one is a semantic space based on simple word co-occurrence, whereas the other is a topic model based on Latent Dirichlet Allocation (LDA, Blei et al., 2003). There are a number of differences between these approaches. In particular, the type of distributional information exploited by each model is different. In the case of the simple semantic space the distribution of a word is represented in terms of its co-occurrence with a set of context words, whereas the LDA model looks at distribution across documents. Moreover, the components of the vectors in the simple semantic space correspond directly to those context words, whereas those of the LDA model correspond to latent factors, inferred probabilistically, which do not map one-to-one onto document contexts.

We chose these two methods as representing distinct approaches to building such models, yet also being relatively simple and popular. A diversity of other methods are of course available, but attempting an exhaustive survey would be prohibitive and produce more data than could be meaningfully interpreted. Instead we focus on these 
two simple approaches as a test of whether the questions we want to ask produce meaningful answers and also as a basis for further investigation.

One specific option we do not explore is that of using syntactic relations to define our contexts. The contexts we use here are windows and documents, which act as bags-of-words. Syntactic models (Grefenstette, 1994; Lin, 1998; Padó and Lapata, 2007) take into account the syntactic relations between words in defining their contexts, and this can help improve their performance. However, combining these word representations to handle phrases is complicated by the fact that their contexts will often be disjoint, limiting the potential for combining them. So, for example, the syntactic relations available to a verb are substantially different to those of a noun, and representations based on syntactic contexts for these words will have very different sets of active components. The question of how to reconcile and integrate these disparate sets of components remains open. Instead, we work with simpler bag-of-words contexts, which do not suffer from this problem.

\subsection{Corpora}

The distributional models we employed in this thesis were based on two sources of data. One was the written part of the British National Corpus (BNC) and the other was the Brown Laboratory for Linguistic Information Processing (BLLIP) corpus. The written part of the BNC contains about 90 million words drawn from a mixture of samples of fiction and non-fiction published in a variety of sources such as newspapers, leaflets and books. All the texts were written in British English in the second half of the 20th century. The BLLIP corpus contains 30 million words from news articles written in American English and published in the Wall Street Journal between 1987 and 1989.

We use these data sources in Chapters 5, 6 and 7 on two distinct compositional applications. The first was the task of simulating human similarity judgements on short phrases and the second was the task of language modelling. The differences in 
these tasks determined the choice of data and how that data was handled. The phrase similarity judgements, being gathered mainly from native speakers of British English, were modelled in terms of the BNC. In addition, this corpus was lemmatised to focus on the semantic content of the words as opposed to their surface realisation. Language modelling, on the other hand, is typically carried out on the WSJ, and so we used the BLLIP corpus on this task. Lemmatisation in this case is not appropriate, as the goal is to predict the actual sequence of words in a text, as opposed to just the semantic content. Instead, standard language modelling pre-processing was applied to the data with numbers replaced by the symbol $\langle$ num $\rangle$, a vocabulary of 20,000 words chosen and the remaining tokens replaced with $\langle$ unk $\rangle$.

Both corpora consist of a number of documents, each containing text from a single source. This internal structure is slightly different for each corpus, with the BNC consisting of 4,054 documents, and BLLIP 94,423. In the case of the BNC we used all these documents as a training set, as the models built on this corpus would be evaluated on a distinct set of data gathered in human experiments. In the case of BLLIP, for the purposes of language modelling, we randomly split the corpus into an 85,455 document training set consisting of 38,521,346 words, a 4,456 document development set of 2,095,964 words and a 4,511 document test set of 2,074,446 words ${ }^{1}$. We further randomly sampled sentences from these latter two sets to produce smaller development and test sets, of 52,070 and 50,006 words respectively, for the evaluation of language models that could not be run feasibly on the larger datasets.

\subsection{Acquiring Distributional Counts}

Constructing distributional representations from this data requires that the idea of context be given a concrete definition which allows us to compile counts for target words

\footnotetext{
${ }^{1}$ The development and test sets were drawn from the year 1987 to avoid overlap with the Penn Treebank, which we used to train Roark's (2001) parser. This ensured the development and test sets were entirely distinct from all the training data.
} 
across these contexts. We evaluate two alternatives here: a simple semantic space (SSS) and a model based on Latent Dirichlet Allocation (LDA). The simple semantic space defines contexts in terms of co-occurence with a set of context words, whereas the contexts of LDA are latent variables, known as topics. We compiled counts for each target word in each context, and then transform these counts (as described in Section 3.4) to produce vectors which allow the measurement of similarity between representations.

\subsubsection{Simple Semantic Space}

In the simple semantic space, the distribution of a target word was assessed in terms of its co-occurrence with a set of context words. Co-occurrence in our case meant the presence of a context word token within a five word span either side of a target word token. Context words were defined as common content words, with each context word corresponding to a component of the resulting vector. Thus, the number of context words defines the dimensionality of the vector space, and we considered spaces ranging from 50 to 500,000 dimensions. In each space of dimensionality $N$, the context words were the $N$ most frequent content words. This defined a set of raw counts, $S_{c t}$, of the number times target word $t$ occurred with context word $c$ within the sample corpus. These counts were then smoothed by adding a small quantity, $\beta=0.01$, to produce the frequencies used in the generation of the semantic representations.

$$
\operatorname{freq}_{c t}=S_{c t}+\beta
$$

\subsubsection{Latent Dirichlet Allocation}

In our LDA representations, topics play the role that content words played in the semantic space. They define the contexts across which words are distributed, and each component of the semantic representation corresponds to a particular topic. However, unlike content words they cannot be observed directly in the data. Instead they are 
hidden variables which arise in a generative model of the distribution of words across documents.

As explained in Chapter 2, Latent Dirichlet Allocation (Blei et al., 2003) models the relationship between words and documents in terms of topics, with each document being a mixture of topics and each topic being a unigram distribution over words. The variations in vocabulary across documents are thus explained by variation in topic content, with topics acting like soft clusterings of words.

To create our representations, we use the implementation described by Phan et al. $(2008)^{2}$. In this approach, both the document topic mixtures and the topic word distributions are controlled by Dirichlet priors, having parameters $\alpha$ and $\beta$ respectively, which are chosen before training begins. This choice then defines the probability, $P(D \mid \alpha, \beta)$, of a collection, $D$, of $M$ documents, $d$, to be:

$$
\int P(\Phi \mid \beta) \prod_{d=1}^{M} \int P\left(\theta_{d} \mid \alpha\right)\left(\prod_{n=1}^{N_{d}} \sum_{c_{d n}} P\left(c_{d n} \mid \theta_{d}\right) P\left(w_{d n} \mid c_{d n}, \Phi\right)\right) d \Phi d \theta_{d}
$$

where $w_{d n}$ is the $n$th word of document $d, c_{d n}$ is the topic which generated that word, $\theta_{d}$ is the topic distribution of document $d$ and $\Phi$ is a matrix of word probabilities given topics.

Training of this model is then based on a Gibbs sampling procedure, which allows us to estimate the $\theta_{d}$ and $\Phi$ for a given document collection. This algorithm iteratively approximates the target distribution, producing samples of its variables, including, here, the hidden topics $c$. The basic idea is that each variable is sampled from its distribution conditioned on the current values of the remaining variables. When this process eventually converges to the correct joint distribution, the hidden variable assignments can be used to infer the document topic mixtures and topic word distributions.

Given a set of such samples, consisting of a topic assignment for each word in each document, Equation 3.2 allows the derivation of posterior distributions for $\theta$ and $\Phi$.

\footnotetext{
${ }^{2}$ Available at http://gibbslda.sourceforge. net/.
} 
The expected value of $\theta_{d c}$, the probability of topic $c$ in document $d$, is then given by:

$$
\theta_{d c}=\frac{S_{d c}+\alpha}{\sum_{c} S_{d c}+\alpha}
$$

where $S_{d c}$ is the number of times topic $c$ occurs in document $d$ among the samples. Similarly, the expected value of $\Phi_{c t}$, the probability of word $t$ given topic $c$, is given by:

$$
\Phi_{c t}=\frac{S_{c t}+\beta}{\sum_{t} S_{c t}+\beta}
$$

where $S_{c t}$ is the number of times topic $c$ occurs with word $t$. In effect, the contribution of the dirichlet priors is to smooth the raw sample counts by adding a small amount to each, and it is these smoothed counts we use to construct the semantic vectors.

$$
\text { freq }_{c t}=S_{c t}+\beta
$$

In our experiments we set $\alpha$ and $\beta$ to 0.1 , and 0.01 respectively. We excluded all function words from the training data and experimented with models ranging from 10 to 1,000 topics. For each of these models we performed 150 iterations of the sampling procedure to allow for covergence of the algorithm before drawing the samples used to construct the semantic vectors.

\subsection{Defining the Space}

Having acquired smoothed frequencies for the distribution of words across contexts as described in the previous section, we now need to construct spatial representations from them. This requires two choices to be made: how to transform counts to produce vector components and how to measure the similarity between those vectors.

Table 3.1 lists a number of approaches to defining vector components, drawn from the survey of Bullinaria and Levy (2007). The Conditional Probability components are based simply on the probability of a context, $c$, given a target word, $t$. By conditioning on the target word, as opposed to using raw frequencies or joint probabilities, 
differences in the underlying frequencies of target words are ignored. However, the effects of differences in the underlying frequencies of the contexts remain. The Ratio of Probabilities components take these differences into account to produce a measure of how much greater or lesser than expected the context counts are. Pointwise Mutual Information is essentially the log of this ratio, and measures the contribution of each context-target pair to the overall mutual information between contexts and targets. In cases where a context occurs less than expected with a given target this measure is negative. The Positive Pointwise Mutual Information measure replaces these negative values with zeroes, keeping only the positives. There are a number of reasons why this may be beneficial. First, negatives arise where the actual number of counts is less than expected, and since this will often be due to sparsity, these values may not be reliable. Second, the contexts which have positive values are those which occur most strongly with the target word, and so these are probably the values most relevant to its meaning. Finally, the lower bound of these negatives is minus infinity, which may result in them having undue influence in the representation.

The typical method of quantifying the similarity of two such vectors, is to use the cosine of the angle between them.

$$
\cos (\mathbf{u}, \mathbf{v})=\frac{\mathbf{u} \cdot \mathbf{v}}{\sqrt{\mathbf{u} \cdot \mathbf{u}} \sqrt{\mathbf{v} \cdot \mathbf{v}}}
$$

We apply this measure to the vectors defined by the formulas in Table 3.1.

Weeds (2003) considers various other similarity measures defined on simple conditional probability vectors. We implemented a number of these measures, which are listed in Table 3.2. The L1 city block metric measures the distance between two points when the path travelled consists of orthogonal segments along basis directions. Pearson's $r$ measures the linear correlation between two sets of values and this is the measure of similarity listed as Product Moment Correlation. The Jensen-Shannon Divergence is a symmetrised version of the Kullback-Leibler divergence, which can be thought of as an information theoretic measure of how substitutable one distribution is for another. The Confusion Probability also measures the ability of one distribution 


\begin{tabular}{|c|c|c|}
\hline Name & Description & Components \\
\hline cond & Conditional Probabilities & $p(c \mid t)=\frac{\text { freq } c t}{\text { freq }}$ \\
\hline ratio & Ratio of Probabilities & $\frac{p(c \mid t)}{p(c)}=\frac{\text { freq }_{c t} \text { freq }_{\text {total }}}{\text { freq }_{c} \text { freq }}$ \\
\hline pmi & Pointwise Mutual Information & $\log \left(\frac{\text { freq }_{\text {ct }} \text { freq }_{\text {total }}}{\text { freq }_{c} \text { freq }_{t}}\right)$ \\
\hline pospmi & Positive PMI & $\max \left(0, \log \left(\frac{\text { freq }_{\text {ct }} \text { freq }_{\text {total }}}{\text { freq }_{c} \text { freq }}\right)\right)$ \\
\hline
\end{tabular}

Table 3.1: Definitions of vector components in terms of the ferquency, $f r e q_{c t}$, of the tar-

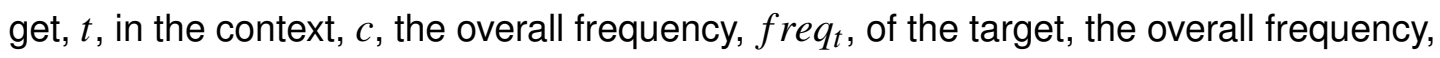
freq $_{c}$, of the context, $c$, and the total frequency, freqtotal, of all words.

to be substituted for another, but produces a probability value rather than a number of bits. Hindle's Measure captures the extent to which the high mutual information contexts for a pair of targets are in concordance. It is worth noting that the L1 and Jensen-Shannon measures are distance measures, in that they produce large values for dissimilar representations, in contrast to the other measures which are similarity measures, producing the highest values for identical representations.

In addition to varying these component and similarity measure definitions, we also investigated the effect of the dimensionality of the space, by varying the size of the context set (words for the simple semantic space, topics for the LDA model) as described in the previous section. 


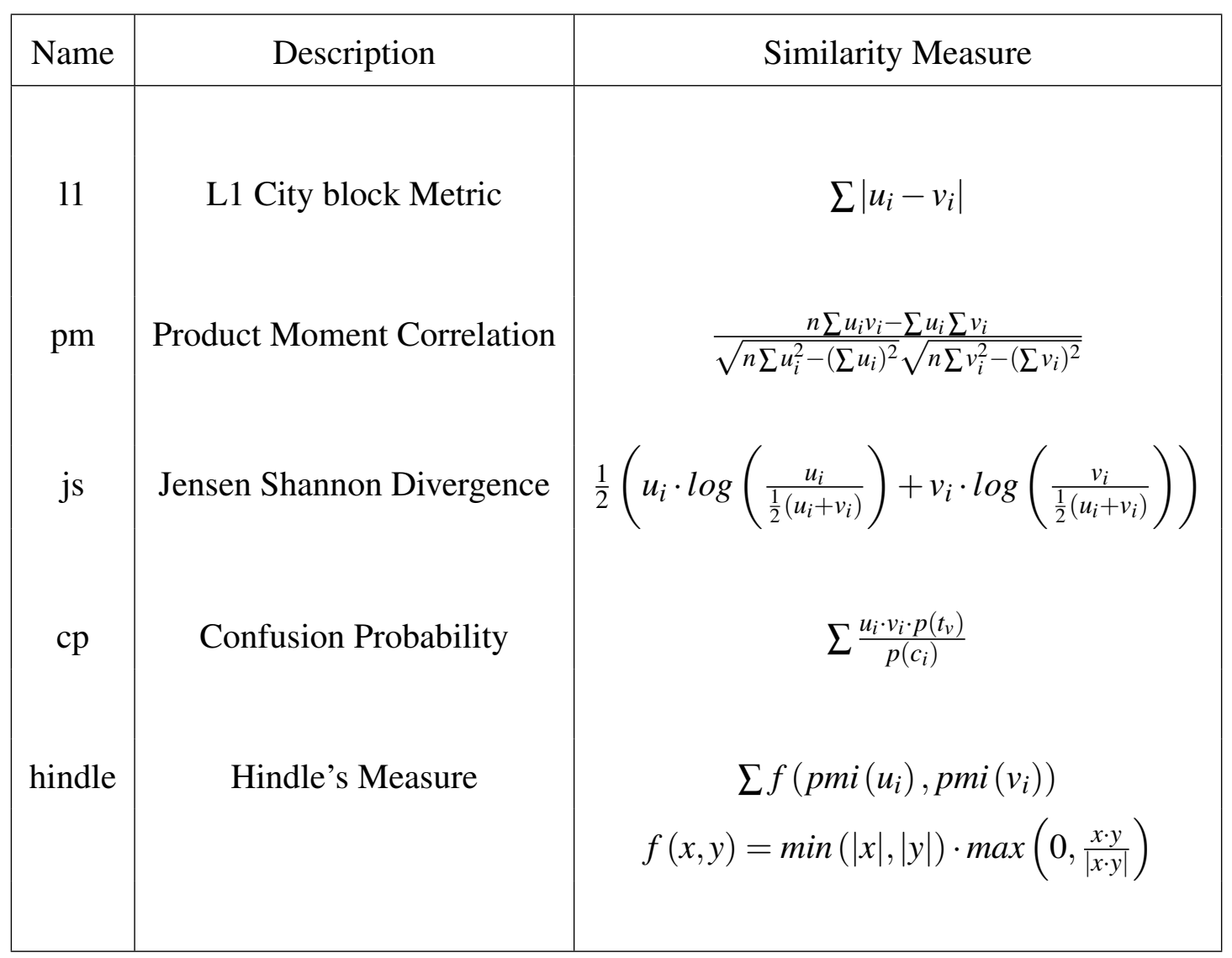

Table 3.2: Definitions of similarity measures for a pair of vectors $u$ and $v$. 


\subsection{Evaluation}

We considered two tasks for evaluating the semantic representations described in the previous sections. The first was predicting similarity judgements and the second was identifying synonyms. The fundamental semantic information that can be extracted from distributional models are similarities between pairs of representations and the application and evaluation of these models is typically made to a problem in which such information is relevant. Priming, for example, has been modelled using distributional representations (Landauer et al., 1997; Lund and Burgess, 1996) on the assumption that priming strength depends on the similarity of target and prime. The tasks we have chosen evaluate this ability to capture similarity relations fairly directly. In the first task, we quantify the ability of these models to predict similarity ratings in terms of the correlation between model predictions and human judgements. In the second task, we measure their ability to identify the synonym of a test word from among a set of alternatives by choosing the most similar option. Both tasks therefore probe the validity of the similarities these models assign to pairs of words. However, the synonym identification task is based on the ability of the models to identify the most similar items and does not evaluate the correctness of predictions at the low similarity end of the spectrum. In contrast, the similarity prediction task evaluates the performance across the whole of this range. As a result, we can expect that our models may give differing results on the two tasks.

\subsubsection{Predicting Similarity Judgements}

The ability of the semantic spaces described in the previous sections to predict human similarity judgements was evaluated against the WordSim353 test collection (Finkelstein et al., 2002). This contains similarity judgements made by 13 to 16 participants on a set of 353 word pairs, which contains, as a subset, the smaller collection of word pairs used by Miller and Charles (1991). Evaluation against this larger collection should 
produce more reliable results than using the more restricted sets of Rubenstein and Goodenough (1965) or Miller and Charles (1991).

For each candidate space, we calculated the similarity of the vectors representing pairs of words in the WordSim353 collection and measured the correlation of these model similarities with the human similarity judgements. To avoid making unnecessary assumptions about the form of the relationship between the ratings and the model predictions, we use a non-parametric correlation measure, Spearman's $\rho$. We interpret the magnitude of these correlations as reflecting the ability of our representations to predict the similarity judgements. However, while most of our models produce predicted similarities with the same ordering as the human similarities (high values correspond to highly similar items), the L1 and Jensen-Shannon measures are distance measures and produce values in the opposite ordering. Consequently, the correlations for these models are typically negative and our comparison of correlation strengths needs to be based on the absolute value of $\rho$.

The correlations of the predicted similarities with the WordSim353 ratings are found in Tables 3.3, 3.4, 3.5 and 3.6. Starting with the simple semantic space constructed on the BNC, Table 3.3 reveals that the ratio components produce the highest correlations for all dimensions. In fact, that column contains the only values greater than 0.30 , with only the pospmi, cond, $p m$ and $c p$ models achieving correlations over 0.20 . The values of ratio exceed 0.30 at 2,000 dimensions and then remain above that value until 500,000 dimensions, peaking at around 10,000 to 100,000 . In contrast, hindle fails to pass 0.10 and is the worst performing model.

The correlations of the LDA model constructed on the same data are reported in Table 3.4. Here all the approaches produce much more effective representations with many attaining correlations of 0.50 and over. The cond and ratio columns reach this level at 100 dimensions and the pospmi and pm models achieve marginally higher values for similar sizes. In fact, pospmi gives the best performance of all models, with a value of 0.54 . Here, hindle demonstrates much higher correlations, performing 


\begin{tabular}{|l|ccccccccc|}
\hline $\operatorname{dim}$ & pmi & pospmi & cond & ratio & 11 & pm & js & cp & hindle \\
\hline 50 & 0.05 & 0.13 & 0.17 & 0.19 & -0.13 & 0.17 & -0.12 & -0.02 & 0.08 \\
100 & 0.04 & 0.13 & 0.20 & 0.22 & -0.17 & 0.20 & -0.15 & -0.01 & 0.05 \\
200 & 0.05 & 0.12 & 0.21 & 0.26 & -0.19 & 0.22 & -0.16 & 0.01 & 0.03 \\
500 & 0.09 & 0.12 & 0.22 & 0.27 & -0.19 & 0.23 & -0.16 & 0.04 & 0.01 \\
1000 & 0.16 & 0.11 & 0.22 & 0.30 & -0.19 & 0.24 & -0.16 & 0.07 & 0.01 \\
2000 & 0.18 & 0.09 & 0.22 & 0.32 & -0.19 & 0.24 & -0.16 & 0.10 & -0.00 \\
5000 & 0.17 & 0.09 & 0.22 & 0.36 & -0.18 & 0.23 & -0.15 & 0.14 & -0.01 \\
10000 & 0.16 & 0.12 & 0.21 & 0.37 & -0.18 & 0.22 & -0.16 & 0.18 & -0.00 \\
20000 & 0.16 & 0.15 & 0.21 & 0.38 & -0.18 & 0.21 & -0.15 & 0.20 & 0.01 \\
50000 & 0.15 & 0.21 & 0.21 & 0.37 & -0.17 & 0.21 & -0.15 & 0.21 & 0.01 \\
100000 & 0.15 & 0.23 & 0.20 & 0.38 & -0.17 & 0.21 & -0.15 & 0.22 & 0.02 \\
200000 & 0.15 & 0.26 & 0.20 & 0.35 & -0.17 & 0.20 & -0.15 & 0.23 & 0.02 \\
500000 & 0.11 & 0.26 & 0.20 & 0.30 & -0.17 & 0.20 & -0.15 & 0.23 & 0.02 \\
\hline
\end{tabular}

Table 3.3: Correlations of model similarities with human ratings for simple semantic space models constructed on the BNC

\begin{tabular}{|l|ccccccccc|}
\hline $\operatorname{dim}$ & pmi & pospmi & cond & ratio & 11 & pm & js & cp & hindle \\
\hline 10 & 0.38 & 0.34 & 0.32 & 0.35 & -0.32 & 0.33 & -0.33 & 0.14 & 0.32 \\
20 & 0.36 & 0.42 & 0.42 & 0.42 & -0.38 & 0.44 & -0.38 & 0.21 & 0.37 \\
50 & 0.42 & 0.45 & 0.41 & 0.42 & -0.37 & 0.44 & -0.40 & 0.28 & 0.35 \\
100 & 0.41 & 0.54 & 0.50 & 0.50 & -0.47 & 0.52 & -0.49 & 0.35 & 0.40 \\
200 & 0.37 & 0.52 & 0.48 & 0.51 & -0.45 & 0.53 & -0.48 & 0.35 & 0.36 \\
500 & 0.36 & 0.52 & 0.46 & 0.48 & -0.47 & 0.50 & -0.50 & 0.35 & 0.37 \\
1000 & 0.30 & 0.51 & 0.48 & 0.49 & -0.51 & 0.52 & -0.52 & 0.37 & 0.35 \\
\hline
\end{tabular}

Table 3.4: Correlations of model similarities with human ratings for LDA models constructed on the BNC 


\begin{tabular}{|l|ccccccccc|}
\hline $\operatorname{dim}$ & pmi & pospmi & cond & ratio & 11 & pm & js & cp & hindle \\
\hline 50 & 0.10 & 0.12 & 0.13 & 0.13 & -0.10 & 0.13 & -0.10 & 0.00 & 0.08 \\
100 & 0.11 & 0.11 & 0.14 & 0.15 & -0.11 & 0.14 & -0.11 & 0.01 & 0.05 \\
200 & 0.13 & 0.10 & 0.13 & 0.19 & -0.11 & 0.13 & -0.11 & 0.02 & 0.04 \\
500 & 0.15 & 0.08 & 0.11 & 0.18 & -0.11 & 0.11 & -0.11 & 0.03 & 0.03 \\
1000 & 0.14 & 0.07 & 0.10 & 0.17 & -0.11 & 0.10 & -0.12 & 0.04 & 0.02 \\
2000 & 0.12 & 0.07 & 0.10 & 0.20 & -0.12 & 0.10 & -0.13 & 0.07 & 0.01 \\
5000 & 0.10 & 0.09 & 0.11 & 0.25 & -0.14 & 0.11 & -0.13 & 0.12 & 0.02 \\
10000 & 0.09 & 0.10 & 0.10 & 0.25 & -0.14 & 0.10 & -0.12 & 0.14 & 0.03 \\
20000 & 0.08 & 0.13 & 0.10 & 0.26 & -0.13 & 0.10 & -0.11 & 0.16 & 0.04 \\
\hline
\end{tabular}

Table 3.5: Correlations of model similarities with human ratings for simple semantic space models constructed on BLLIP

\begin{tabular}{|l|ccccccccc|}
\hline $\operatorname{dim}$ & pmi & pospmi & cond & ratio & 11 & pm & js & cp & hindle \\
\hline 10 & 0.40 & 0.33 & 0.31 & 0.30 & -0.32 & 0.35 & -0.32 & 0.18 & 0.40 \\
20 & 0.33 & 0.33 & 0.28 & 0.30 & -0.27 & 0.31 & -0.29 & 0.22 & 0.36 \\
50 & 0.35 & 0.44 & 0.41 & 0.41 & -0.38 & 0.43 & -0.41 & 0.34 & 0.43 \\
100 & 0.37 & 0.43 & 0.38 & 0.39 & -0.36 & 0.42 & -0.38 & 0.36 & 0.37 \\
200 & 0.33 & 0.49 & 0.43 & 0.45 & -0.44 & 0.47 & -0.44 & 0.41 & 0.39 \\
500 & 0.31 & 0.52 & 0.46 & 0.47 & -0.45 & 0.49 & -0.46 & 0.44 & 0.42 \\
1000 & 0.25 & 0.47 & 0.43 & 0.43 & -0.39 & 0.44 & -0.39 & 0.42 & 0.40 \\
\hline
\end{tabular}

Table 3.6: Correlations of model similarities with human ratings for LDA models constructed on BLLIP 
comparably to $p m i$ and $c p$.

Turning to the models constructed on the BLLIP corpus, Table 3.5 presents the results for the simple semantic space. Again, the ratio components produce clearly the best performance, with the closest competitor being $c p$, which reaches a value of 0.16 for 20,000 dimensions. The value of ratio, in contrast reaches 0.20 at 2,000 dimensions and then peaks at 0.26 at 20,000. Hindle is again the worst performing model.

Table 3.6 contains much higher correlations for the LDA models, with all models attaining values greater than 0.40 , except pmi. The highest correlation, 0.52 , is produced by pospmi with 500 dimensions. The runners up, cond, ratio, $p m$ and $c p$, all produce very similar results.

\subsubsection{Identifying Synonyms}

We next examined the performance of these models on a TOEFL task (Landauer and Dumais, 1996). This involved identifying the synonym of a given test word from among four alternatives. To do this, we calculated the similarity to each of the alternatives and then chose the closest for each model, and Tables 3.7 to 3.10 give the model accuracies across the 80 items.

Examining the results for the BNC simple semantic space first, Table 3.7 indicates that the $c p$ model produces the highest TOEFL scores of 0.81 , for dimensions of 50,000 context words and over. Next most effective are ratio and pospmi, which achieve levels of 0.68 for around 2,000 to 50,000 dimensions and 0.69 at 100,000 respectively. Scores over 0.60 are also attained by cond, $l 1, \mathrm{pm}$ and $j s$.

The scores for the LDA model on the BNC data are listed in Table 3.8. For this model the values are generally much lower, with the highest being 0.63 for the 1,000 topic model with pospmi components. However, this value is considerably larger than for smaller numbers of topics, and may be just a statistical fluke.

Table 3.9 lists the results for the simple semantic space built on BLLIP. As on the other simple semantic space, here $c p$ produces the best scores, attaining 0.67 with 


\begin{tabular}{|l|ccccccccc|}
\hline dim & pmi & pospmi & cond & ratio & 11 & pm & js & cp & hindle \\
\hline 50 & 0.42 & 0.51 & 0.64 & 0.62 & 0.56 & 0.61 & 0.60 & 0.43 & 0.39 \\
100 & 0.36 & 0.51 & 0.56 & 0.57 & 0.55 & 0.53 & 0.55 & 0.51 & 0.39 \\
200 & 0.42 & 0.53 & 0.61 & 0.65 & 0.60 & 0.58 & 0.60 & 0.52 & 0.42 \\
500 & 0.40 & 0.52 & 0.65 & 0.62 & 0.58 & 0.66 & 0.61 & 0.64 & 0.38 \\
1000 & 0.45 & 0.51 & 0.64 & 0.64 & 0.57 & 0.66 & 0.56 & 0.68 & 0.42 \\
2000 & 0.47 & 0.49 & 0.61 & 0.68 & 0.56 & 0.65 & 0.55 & 0.71 & 0.44 \\
5000 & 0.42 & 0.55 & 0.62 & 0.66 & 0.52 & 0.64 & 0.51 & 0.71 & 0.44 \\
10000 & 0.38 & 0.56 & 0.62 & 0.68 & 0.52 & 0.62 & 0.51 & 0.77 & 0.44 \\
20000 & 0.40 & 0.60 & 0.65 & 0.66 & 0.51 & 0.65 & 0.53 & 0.79 & 0.44 \\
50000 & 0.42 & 0.66 & 0.65 & 0.68 & 0.53 & 0.65 & 0.52 & 0.81 & 0.44 \\
100000 & 0.42 & 0.69 & 0.65 & 0.66 & 0.53 & 0.65 & 0.52 & 0.81 & 0.44 \\
200000 & 0.45 & 0.68 & 0.65 & 0.65 & 0.53 & 0.65 & 0.52 & 0.81 & 0.44 \\
500000 & 0.40 & 0.65 & 0.65 & 0.57 & 0.53 & 0.65 & 0.51 & 0.81 & 0.44 \\
\hline
\end{tabular}

Table 3.7: Proportion of TOEFL items correct for simple semantic space models constructed on the BNC

\begin{tabular}{|l|ccccccccc|}
\hline $\operatorname{dim}$ & pmi & pospmi & cond & ratio & 11 & pm & js & cp & hindle \\
\hline 10 & 0.40 & 0.41 & 0.42 & 0.40 & 0.42 & 0.41 & 0.44 & 0.44 & 0.41 \\
20 & 0.44 & 0.42 & 0.40 & 0.38 & 0.48 & 0.38 & 0.40 & 0.37 & 0.49 \\
50 & 0.45 & 0.42 & 0.45 & 0.45 & 0.45 & 0.42 & 0.47 & 0.45 & 0.48 \\
100 & 0.45 & 0.49 & 0.55 & 0.58 & 0.58 & 0.52 & 0.52 & 0.48 & 0.53 \\
200 & 0.44 & 0.53 & 0.48 & 0.53 & 0.49 & 0.52 & 0.53 & 0.48 & 0.51 \\
500 & 0.36 & 0.47 & 0.52 & 0.53 & 0.56 & 0.53 & 0.56 & 0.45 & 0.49 \\
1000 & 0.41 & 0.63 & 0.56 & 0.53 & 0.62 & 0.55 & 0.59 & 0.48 & 0.58 \\
\hline
\end{tabular}

Table 3.8: Proportion of TOEFL items correct for LDA models constructed on the BNC 


\begin{tabular}{|l|ccccccccc|}
\hline $\operatorname{dim}$ & pmi & pospmi & cond & ratio & 11 & pm & js & cp & hindle \\
\hline 50 & 0.48 & 0.45 & 0.40 & 0.52 & 0.37 & 0.40 & 0.48 & 0.40 & 0.33 \\
100 & 0.46 & 0.39 & 0.36 & 0.52 & 0.34 & 0.34 & 0.45 & 0.39 & 0.30 \\
200 & 0.43 & 0.39 & 0.37 & 0.45 & 0.39 & 0.36 & 0.40 & 0.49 & 0.42 \\
500 & 0.40 & 0.37 & 0.40 & 0.43 & 0.40 & 0.40 & 0.42 & 0.55 & 0.36 \\
1000 & 0.42 & 0.43 & 0.39 & 0.58 & 0.40 & 0.39 & 0.42 & 0.54 & 0.40 \\
2000 & 0.40 & 0.45 & 0.45 & 0.52 & 0.43 & 0.45 & 0.45 & 0.58 & 0.37 \\
5000 & 0.39 & 0.46 & 0.46 & 0.54 & 0.45 & 0.46 & 0.45 & 0.63 & 0.40 \\
10000 & 0.40 & 0.55 & 0.46 & 0.60 & 0.40 & 0.46 & 0.42 & 0.67 & 0.42 \\
20000 & 0.45 & 0.54 & 0.46 & 0.52 & 0.48 & 0.46 & 0.45 & 0.64 & 0.40 \\
\hline
\end{tabular}

Table 3.9: Proportion of TOEFL items correct for simple semantic space models constructed on BLLIP

\begin{tabular}{|l|ccccccccc|}
\hline $\operatorname{dim}$ & pmi & pospmi & cond & ratio & 11 & pm & js & cp & hindle \\
\hline 10 & 0.50 & 0.42 & 0.39 & 0.44 & 0.47 & 0.44 & 0.47 & 0.42 & 0.39 \\
20 & 0.42 & 0.47 & 0.50 & 0.47 & 0.47 & 0.50 & 0.50 & 0.39 & 0.44 \\
50 & 0.42 & 0.42 & 0.42 & 0.50 & 0.36 & 0.42 & 0.39 & 0.47 & 0.44 \\
100 & 0.42 & 0.56 & 0.50 & 0.44 & 0.47 & 0.50 & 0.53 & 0.50 & 0.50 \\
200 & 0.42 & 0.42 & 0.50 & 0.44 & 0.47 & 0.56 & 0.50 & 0.47 & 0.44 \\
500 & 0.36 & 0.44 & 0.53 & 0.56 & 0.44 & 0.53 & 0.47 & 0.50 & 0.44 \\
1000 & 0.28 & 0.53 & 0.47 & 0.53 & 0.42 & 0.42 & 0.50 & 0.56 & 0.56 \\
\hline
\end{tabular}

Table 3.10: Proportion of TOEFL items correct for LDA models constructed on BLLIP 
10,000 context words. Second place goes to ratio, with a score of 0.60 for the same size of space. Of the remaining models, only pospmi exceeds 0.50 .

Finally, the performance of the LDA models using the BLLIP corpus is again worse than the simple semantic space, the highest score being 0.56 . This is achieved by pospmi, at 100 topics, ratio, at 500 topics, pm, at 200, and cp and hindle, both at 1,000.

\subsubsection{Discussion}

The results of the preceding evaluations reveal that the effectiveness of any approach to constructing distributional representations is to a great extent dependent on the particular corpus and task it is applied to. Hindle's similarity measure, for example, performed extremely poorly on the similarity task for the simple semantic space. In contrast, on the synonymy task, using the same space, its performance was much more competitive. Similarly, the LDA approach appeared to achieve better performance than the simple semantic space on the similarity task, whereas this relationship was reversed on the synonymy task.

As discussed, one of the major differences between the two tasks is that whereas predicting similarity ratings requires a good representation across the whole range of similarities, the synonym identification task is focused on the highly similar end of the spectrum. We might expect LDA to be weaker on such a fine-grained task for a number of reasons. Firstly, the LDA representations are typically of a lower dimensionality and they are also sparser in comparison to the high-dimensional, distributed representations of the simple semantic space. This almost inevitably means the LDA representations are coarser. Secondly, the contexts relevant to LDA are whole documents, in comparison to the five word context window of the simple semantic space. Thus, the distributional information available to LDA is probably coarser too. This coarseness of it semantic representations, probably helps to explain LDA's weaker performance on the synonym identification task.

Given these variations in performance between the two evaluations, identify a sin- 
gle optimal representation is not possible. Instead, we wish to select an approach which is likely to be robust, in the sense that it performs well across tasks and data and is less vulnerable to the effects of sparsity. The ratio model gives good results in all the evaluations. On the similarity task for the simple semantic space it clearly outperforms the alternatives, while elsewhere it remains a strong contender. We therefore select the ratio definition of vector components for both the simple semantic space and the LDA model. The remaining choice is the number of dimensions for each space. For the simple semantic space, 2,000 dimensions appears to contain enough information about the contexts a word occurs in to ensure a strong performance while avoiding an overly large and sparse space. In the case of LDA, we chose 100 topics to achieve the balance between representational capacity and sparsity.

\subsection{Conclusions}

In this chapter, we evaluated a range of distributional representations constructed on the BNC and BLLIP corpora. We selected two models for each data source: a simple semantic space and a model based on Latent Dirichlet Allocation. This choice was motivated by the ability to produce robust and effective semantic representations of individual words, as measured on two separate tasks.

In Chapters 5, 6 and 7 we will use these word level representations in the evaluation of composition models for the construction of representations of phrases and sentences. Chapter 5 essentially extends the similarity prediction task to phrase level similarities, gathering similarity judgements for phrases and modelling these in terms of distributional models of composition. Chapter 6 then evaluates these models of semantic composition in the setting of language modelling and Chapter 7 tests the relevance of these models to the cognitive processes of language comprehension in terms of their ability to predict eye-movements during reading.

However, before carrying out these evaluations, we need to identify which compo- 
sitional models will be evaluated. The space of possible functions for combining two, or more, constituent vectors into one is too large to be meaningfully surveyed in its entirety. In the following Chapter, we will propose a framework for such vector composition, which will allow us to handle this diversity. In addition to discussing existing proposals for vector composition within this scheme, we will propose novel functions which arise naturally within our framework. 


\section{Chapter 4}

\section{A Framework for Vector Composition}

In Chapter 2, we discussed the general nature of semantic composition and described some of the particular functions which have been applied to build representations of compositional structures in distributional models of semantics. Given the great variety of functions that could be potentially exploited as a model of composition, we require some means to get an overview of vector composition and compare the various alternatives rather than just consider individual proposals in isolation. In this chapter we outline a general framework for vector composition functions and consider some of the choices that lead to specific instantiations of composition models.

\subsection{Preliminaries}

Our aim is to construct vector representations for phrases and sentences. We assume that constituents are represented by vectors which subsequently combine in some way to produce a new vector. We can think of this process as being based on a function of the parts and the syntactic structure in which they are embedded, along with any additional background knowledge (see the discussion in Section 2.3). This provides the basic framework in which we will consider vector composition:

$$
\mathbf{p}=f(\mathbf{u}, \mathbf{v}, R, K)
$$




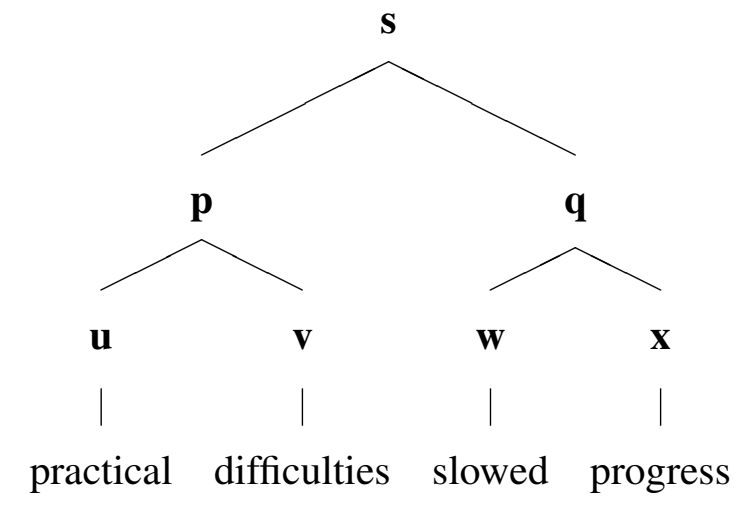

Figure 4.1: Example of composition operating over parse trees.

This equation takes a pair of vectors $\mathbf{u}$ and $\mathbf{v}$ and combines them into a single representation $\mathbf{p}$ of their composition, based on the syntactic relation, $R$ between them, and some additional background knowledge, $K$. Compositional structures consisting of more than two words can also be handled in this formulation by applying Equation 4.1 recursively. If we assume that the parse trees are derived from a grammar in Chomsky Normal Form (Hopcroft and Ullman, 1979), then each binary node involves composing a pair of constituents. For example Figure 4.1 depicts this composition process for the sentence practical difficulties slowed progress. Initially, practical and difficulties are composed into $\mathbf{p}$, and slowed and progress into $\mathbf{q}$. The final sentence representation, $\mathbf{s}$, is the composition of the pair of phrase representations $\mathbf{p}$ and $\mathbf{q}$.

For this process to succeed, composition must produce a single representation of its constituents which can then be used in further processing, including composition with other representations. This is distinct from the related problem of how to represent the semantics of an individual word in context. For example, given the phrase piggy bank we might wish to update the representations of the individual constituents, piggy and bank, to reflect the particular senses in which each is being used in this context. Representation of their composition, however, requires that the constituents are combined, somehow, into a single representation of the whole phrase. This is crucial in enabling the recursive application of Equation 4.1 as illustrated in Figure 4.1. 
Our general framework allows for composition to be dependent on the syntactic relations among the constituents being composed. Proper treatment of this dependence may require that our representation of syntactic structure takes into account the full argument structure, rather than just the limited relation between two constituents. For example, although boy, football and window are all subjects of the sentences in Example (1), they arguably bear distinct semantic roles which ought to be reflected in the compositional process. Thus, the way in which a verb subject is composed is dependent on what other arguments of the verb are present and the syntax term, $R$, in Equation 4.1 must therefore contain this wider information, e.g., whether the verb is being used transitively or not.
(1) a. The boy broke the window with a football
b. A football broke the window
c. The window broke

It is also worth noting that this process only produces well defined results for constituents with a complete syntactic structure. Non-constituents, such as the sentence prefix practical difficulties slowed, are not given a representation in this approach. Although Equation 4.1 can be applied to practical difficulties to produce a representation for this phrase, it does not tell us how to integrate this with slowed. Until the object of this verb is known we cannot compositionally create a representation of the verb phrase which would then be available for composition with the subject phrase. Thus, incrementality is a problem for our framework, as it necessarily involves handling such non-constituent sentence prefixes.

One way to enable the handling of partial stuctures would be to throw away the dependence on syntax and word order entirely. That is we could revert to the formulation $\mathbf{p}=f(\mathbf{u}, \mathbf{v})$, without any dependence on syntax and where $f()$ is a symmetric function, i.e., is invariant when $\mathbf{u}$ and $\mathbf{v}$ are swapped. Now the combination of vectors to form a representation of the whole can be done in any order without changing the result. That 
is, we treat the structure as a bag of words, and from this perspective a non-constituent is just another bag of words which can be handled in the same manner.

This solution is clearly a gross simplification and we might prefer to preserve the dependence on syntax, while extending our approach to handle partial trees. There is certainly evidence that interpretation proceeds word by word, with syntactic and semantic information being integrated into an incremental representation as it becomes available (Tanenhaus et al., 1995; Marslen-Wilson, 1973; Konieczny, 2000). However, this is potentially a more complex problem than simply representing complete constituents, and we will not attempt to solve it in this thesis.

Instead, we focus here on the problem of how to combine vectors to create representations of complete constituents. In the next section, we examine specific instantiations of our framework, including existing proposals and novel functions. We consider both syntax aware functions, which treat the components asymmetrically, and also simpler symmetric functions, which show no dependence on syntax. In subsequent chapters these functions will be used to model similarity ratings for phrases, to enhance $n$-gram language models, and to model readings times captured in eye-tracking experiments. In the case of the last two applications we will use only the symmetric functions because of their suitability for incremental processing.

\subsection{Composition Functions}

To make the vector composition problem more concrete, consider constructing a representation $\mathbf{p}$ for the phrase practical difficulty from the vectors $\mathbf{u}$ and $\mathbf{v}$ representing the constituents practical and difficulty, respectively. Hypothetical vectors for these constituents are illustrated in Figure 4.2. This simplified semantic space ${ }^{1}$ will serve to illustrate examples of the composition functions we consider in this section.

Equation 4.1 above defines a wide class of composition functions which might be

\footnotetext{
${ }^{1}$ The space has only five dimensions; the matrix cells denote the co-occurrence of the words practical and difficulty with breathing, creative, and so on.
} 


\begin{tabular}{l|ccccc} 
& breathing & creative & economic & approach & joke \\
\hline practical & 0 & 6 & 2 & 10 & 4 \\
difficulty & 1 & 8 & 4 & 4 & 0
\end{tabular}

Figure 4.2: A hypothetical semantic space for practical and difficulty.

applied to this task. To derive specific models from this general framework requires the identification of appropriate constraints that narrow the space of functions being considered. To begin with, we will ignore $\mathrm{K}$ so as to explore what can be achieved in the absence of any background or world knowledge. While background knowledge undoubtedly contributes to the compositional process, and resources like WordNet (Fellbaum, 1998) may be used to provide this information, from a methodological perspective it is preferable to understand the fundamental processes of how representations are composed before trying to understand the interaction between existing representations and those under construction. As far as the syntactic relation $R$ is concerned, we can proceed by investigating one such relation at a time, thus removing any explicit dependence on $R$, but allowing the possibility that we identify distinct composition functions for distinct syntactic relations.

Another particularly useful constraint is to assume that $\mathbf{p}$ lies in the same space as $\mathbf{u}$ and $\mathbf{v}$. This essentially means that all syntactic types have the same dimensionality. The simplification may be too restrictive as it assumes that verbs, nouns and adjectives are substantially similar enough to be represented in the same space. Clark et al. (2008) suggest a scheme in which the structure of a representation depends on its syntactic type, such that, for example, if nouns are represented by plain vectors then adjectives, as modifiers of nouns, are represented by matrices. Thus an adjective maps a given noun representation onto a new vector. More generally, we may question whether representations in a fixed space are flexible enough to cover the full expressivity of language. Intuitively, sentences are more complex than individual phrases and 
this should be reflected in the representation of their meaning. In restricting all representations within a space of fixed dimensions, we are implicitly imposing a limit on the complexity of structures which can be fully represented. Nevertheless, the restriction renders the composition problem computationally feasible. We can use a single method for constructing representations, rather than different methods for different syntactic types. In particular, constructing a vector of $n$ elements is easier than constructing a matrix of $n^{2}$ elements. Moreover, our composition and similarity functions only have to apply to a single space, rather than a set of spaces of varying dimensions.

Given these simplifying assumptions, we can now begin to identify specific mathematical types of functions. For example, if we wish to work with linear composition functions, there are two ways to achieve this. We may assume that $\mathbf{p}$ is a linear function of the Cartesian product of $\mathbf{u}$ and $\mathbf{v}$, giving an additive class of composition functions:

$$
\mathbf{p}=\mathbf{A} \mathbf{u}+\mathbf{B} \mathbf{v}
$$

where $\mathbf{A}$ and $\mathbf{B}$ are matrices which determine the contributions made by $\mathbf{u}$ and $\mathbf{v}$ to $\mathbf{p}$.

Or, we can assume that $\mathbf{p}$ is a linear function of the tensor product of $\mathbf{u}$ and $\mathbf{v}$, giving a multiplicative class of composition functions:

$$
\mathbf{p}=\mathbf{C u v}
$$

where $\mathbf{C}$ is a tensor of rank 3, which projects the tensor product of $\mathbf{u}$ and $\mathbf{v}$ onto the space of p. (Appendix A discusses in more detail the linear algebra which is used in this section.)

Linearity is very often a useful assumption because it constrains the problem considerably. However, this usually means that the solution arrived at is an approximation to some other, non-linear, structure. Going beyond the linear class of multiplicative functions, we will also consider some functions which are quadratic in $\mathbf{u}$, having the general form:

$$
\mathbf{p}=\text { Duuv }
$$

where $\mathbf{D}$ is now a rank 4 tensor which projects the product uuv onto the space of $\mathbf{p}$. 
Within the additive model class (Equation 4.2), the simplest composition function is vector addition:

$$
\mathbf{p}=\mathbf{u}+\mathbf{v}
$$

So, according to Equation 4.5, the addition of the two vectors representing practical and difficulty would be practical + difficulty $=\left[\begin{array}{lllll}1 & 14 & 6 & 14 & 4\end{array}\right]$. This model assumes that composition is a symmetric function of the constituents; in other words, the order of constituents essentially makes no difference. While this might be reasonable for certain structures, a list perhaps, a model of composition based on syntactic structure requires some way of differentiating the contributions of each constituent.

Kintsch (2001) attempts to model the composition of a predicate with its argument in a manner that distinguishes the role of these constituents, making use of the lexicon of semantic representations to identify the features of each constituent relevant to their combination. Specifically, he represents the composition in terms of a sum of predicate, argument and a number of neighbors of the predicate.

$$
\mathbf{p}=\mathbf{u}+\mathbf{v}+\sum_{i} \mathbf{n}_{i}
$$

Considerable latitude is allowed in selecting the appropriate neighbors. Kintsch (2001) considers only the $m$ most similar neighbors to the predicate, from which he subsequently selects $k$, those most similar to its argument. So, if in the composition of practical with difficulty, the chosen neighbor is problem, with problem $=\left[\begin{array}{lllll}2 & 15 & 7 & 9 & 1\end{array}\right]$, then this produces the representation practical + difficulty + problem $=\left[\begin{array}{lllll}3 & 29 & 13 & 23 & 5\end{array}\right]$.

This composition model draws inspiration from the construction-integration model Kintsch (1988), which was originally based on symbolic representations, and introduces a dependence on syntax by distinguishing the predicate from its argument. In this process the selection of relevant neighbors for the predicate plays a role similar to the integration of a representation with existing background knowledge in the original construction-integration model. Here, background knowledge takes the form of the lexicon from which the neighbors drawn. 
A simpler approach to introducing dependence on the syntactic relation, $R$, is to weight the constituents differentially in the summation.

$$
\mathbf{p}=\alpha \mathbf{u}+\beta \mathbf{v}
$$

This makes the composition function asymmetric in $\mathbf{u}$ and $\mathbf{v}$ allowing their distinct syntactic roles to be recognized. For instance, we could give greater emphasis to heads than other constituents. As an example, if we set $\alpha$ to 0.4 and $\beta$ to 0.6 , then $0.4 \cdot$ practical $=\left[\begin{array}{lllll}0 & 2.4 & 0.8 & 4 & 1.6\end{array}\right]$ and $0.6 \cdot$ difficulty $=\left[\begin{array}{lllll}0.6 & 4.8 & 2.4 & 2.4 & 0\end{array}\right]$, and their sum $0.4 \cdot$ practical $+0.6 \cdot$ difficulty $=\left[\begin{array}{lllll}0.6 & 5.6 & 3.2 & 6.4 & 1.6\end{array}\right]$ represents the phrase practical difficulty.

An extreme form of this differential in the contribution of constituents is where one of the vectors, say $\mathbf{u}$, contributes nothing at all to the combination: ${ }^{2}$

$$
\mathbf{p}=\mathbf{v}
$$

In this case practical difficulty would be simply represented by difficulty $=\left[\begin{array}{lllll}1 & 8 & 4 & 4 & 0\end{array}\right]$. Admittedly the model in (4.8) is impoverished and rather simplistic, however it can serve as a simple baseline against which to compare more sophisticated models.

So far we have considered solely additive composition models. These models blend together the content of the constituents being composed. The contribution of $\mathbf{u}$ in Equation 4.5 is unaffected by its relation to $\mathbf{v}$. It might be preferable to scale each component of $\mathbf{u}$ with its relevance to $\mathbf{v}$, namely to pick out the content of each representation that is relevant to their combination. This can be achieved by using a multiplicative function instead:

$$
\mathbf{p}=\mathbf{u} \odot \mathbf{v}
$$

where the symbol $\odot$ represents multiplication of the corresponding components:

$$
p_{i}=u_{i} \cdot v_{i}
$$

\footnotetext{
${ }^{2}$ The model in (4.8) is equivalent to setting $\alpha=0$.
} 
For this model, practical $\odot$ difficulty $=\left[\begin{array}{lllll}0 & 48 & 8 & 40 & 0\end{array}\right]$ would represent the phrase practical difficulty.

Note that the multiplicative function in (4.9) is still a symmetric function and thus does not take word order or syntax into account. However, Equation 4.9 is a particular instance of the more general class of multiplicative functions (Equation 4.3), which allows the specification of asymmetric syntax-sensitive functions. For example, the tensor product is an instance of this class with $\mathbf{C}$ being the identity matrix.

$$
\mathbf{p}=\mathbf{u} \otimes \mathbf{v}
$$

Where the symbol $\otimes$ stands for the operation of taking all pairwise products of the components of $\mathbf{u}$ and $\mathbf{v}$ :

$$
p_{i, j}=u_{i} \cdot v_{j}
$$

So, the tensor product representation of practical difficulty is:

$$
\begin{array}{cccccc}
0 & 0 & 0 & 0 & 0 \\
6 & 48 & 24 & 24 & 0 \\
\text { practical } \otimes \text { difficulty }= & 2 & 16 & 8 & 8 & 0 \\
10 & 80 & 40 & 40 & 0 \\
4 & 32 & 16 & 16 & 0
\end{array}
$$

Circular convolution is also a member of this class:

$$
\mathbf{p}=\mathbf{u} \circledast \mathbf{v}
$$

where the symbol $\circledast$ stands for a compression of the tensor product based on summing along its transdiagonal elements:

$$
p_{i}=\sum_{j} u_{j} \cdot v_{(i-j)}
$$

Subscripts are interpreted modulo $n$ which gives the operation its circular nature. Circular convolution compresses the matrix in (4.13) into a vector of the same dimension as the constituents: practical $\circledast$ difficulty $=\left[\begin{array}{lllll}116 & 50 & 66 & 62 & 80\end{array}\right]$. 
One reason for choosing such multiplicative functions is that the magnitudes of $\mathbf{u}$ and $\mathbf{v}$ can only affect the magnitude of $\mathbf{p}$, not its direction. In contrast, in additive models, the relative magnitudes of $\mathbf{u}$ and $\mathbf{v}$, can have a considerable effect on both the magnitude and direction of $\mathbf{p}$. This can lead to difficulties when working with the cosine similarity measure, which is itself insensitive to the magnitudes of vectors. For example, if vector definitions are optimized by comparing the predictions from the cosine similarity measure to some gold standard, then it is the directions of the vectors which are optimized, not their magnitudes. Utilizing vector addition as the composition function makes the product of the composition dependent on an aspect of the vectors which has not been optimized, namely their magnitude. Multiplicative combinations avoid this problem, because effects of the magnitudes of the constituents only show up in the magnitude of the product, which has no effect on the cosine similarity measure.

The multiplicative class of functions also allows us to think of one representation as modifying the other. This idea is fundamental in logic-based semantic frameworks (Montague, 1974) where different syntactic structures are given different function types. To see how the vector $\mathbf{u}$ can be thought of as something which modifies $\mathbf{v}$, consider the partial product of $\mathbf{C}$ with $\mathbf{u}$, producing a matrix which we shall call $\mathbf{U}$.

$$
\mathbf{p}=\mathbf{C u v}=\mathbf{U v}
$$

Here, the composition function can be thought of as the action of a matrix, $\mathbf{U}$, representing one constituent, on a vector, $\mathbf{v}$, representing the other constituent. This is related to the approach of Clark et al. (2008) to adjective-noun composition. In their scheme, nouns would be represented by vectors and adjectives by matrices which map the original noun representation to the modified representation. In our approach all syntactic types are simply represented by vectors; nevertheless, we can make use of their insight. Equation 4.16 demonstrates how a multiplicative composition tensor, $C$, allows us to map a constituent vector, $\mathbf{u}$, onto a matrix, $\mathbf{U}$, while representing all words with vectors. 


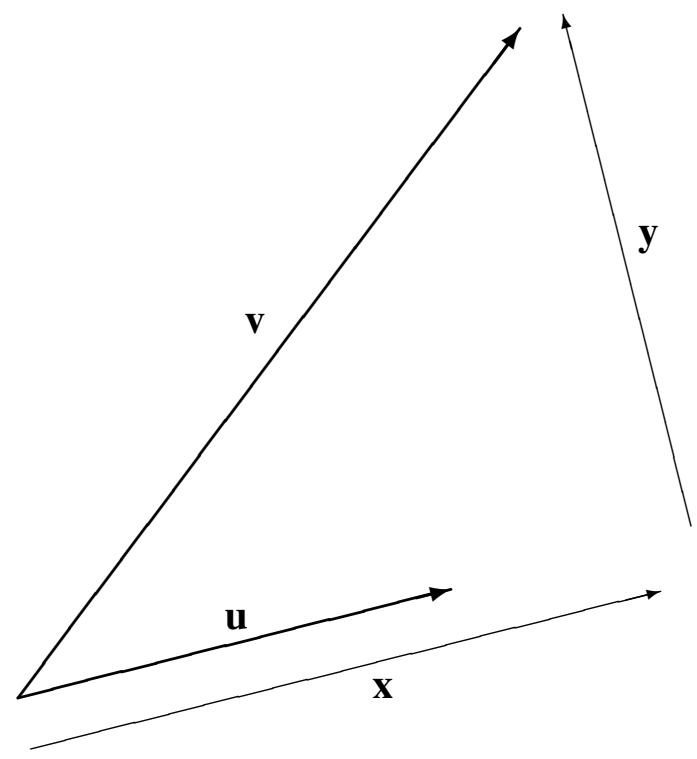

Figure 4.3: Vector $\mathbf{v}$ is decomposed into $\mathbf{x}$, a component parallel to $\mathbf{u}$ and $\mathbf{y}$, a component orthogonal to $\mathbf{u}$.

Putting the simple multiplicative model (see Equation 4.9) into this form yields a matrix, $\mathbf{U}$, whose off-diagonal elements are zero and whose diagonal elements are equal to the components of $\mathbf{u}$.

$$
U_{i j}=0, U_{i i}=u_{i}
$$

The action of this matrix on $\mathbf{v}$ is a type of dilation, in that it stretches and squeezes $\mathbf{v}$ in various directions. Specifically, $\mathbf{v}$ is scaled by a factor of $u_{i}$ along the $i$ th basis.

One drawback of this process is that its results are dependent on the basis used. Ideally, we would like to have a basis independent composition, i.e., one which is based solely on the geometry of $\mathbf{u}$ and $\mathbf{v}^{3}$ One way to achieve basis independence is by dilating $\mathbf{v}$ along the direction of $\mathbf{u}$, rather than along the basis directions. We thus

\footnotetext{
${ }^{3}$ This would allow, for example, the same composition function to be applied both to original vectors and to dimensionality reduced versions, without worrying about how to match the bases of these two spaces.
} 
decompose $\mathbf{v}$ into a component parallel to $\mathbf{u}$ and a component orthogonal to $\mathbf{u}$, and then stretch the parallel component to modulate $\mathbf{v}$ to be more like $\mathbf{u}$. Figure 4.3 illustrates this decomposition of $\mathbf{v}$ where $\mathbf{x}$ is the parallel component and $\mathbf{y}$ is the orthogonal component. These two vectors can be expressed in terms of $\mathbf{u}$ and $\mathbf{v}$ as follows:

$$
\begin{gathered}
\mathbf{x}=\frac{\mathbf{u} \cdot \mathbf{v}}{\mathbf{u} \cdot \mathbf{u}} \mathbf{u} \\
\mathbf{y}=\mathbf{v}-\mathbf{x}=\mathbf{v}-\frac{\mathbf{u} \cdot \mathbf{v}}{\mathbf{u} \cdot \mathbf{u}} \mathbf{u}
\end{gathered}
$$

Thus, if we dilate $\mathbf{x}$ by a factor $\lambda$, while leaving $\mathbf{y}$ unchanged, we produce a modified vector, $\mathbf{v}^{\prime}$, which has been stretched to emphasize the contribution of $\mathbf{u}$ :

$$
\mathbf{v}^{\prime}=\lambda \mathbf{x}+\mathbf{y}=\lambda \frac{\mathbf{u} \cdot \mathbf{v}}{\mathbf{u} \cdot \mathbf{u}} \mathbf{u}+\mathbf{v}-\frac{\mathbf{u} \cdot \mathbf{v}}{\mathbf{u} \cdot \mathbf{u}}=(\lambda-1) \frac{\mathbf{u} \cdot \mathbf{v}}{\mathbf{u} \cdot \mathbf{u}} \mathbf{u}+\mathbf{v}
$$

However, since the cosine similarity function is insensitive to the magnitudes of vectors, we can multiply this vector by any factor we like without essentially changing the model. In particular, multiplying through by $\mathbf{u} \cdot \mathbf{u}$ makes this expression easier to work with:

$$
\mathbf{p}=(\mathbf{u} \cdot \mathbf{u}) \mathbf{v}+(\lambda-1)(\mathbf{u} \cdot \mathbf{v}) \mathbf{u}
$$

In order to apply this model to our example vectors, we must first calculate the dot products practical $\cdot$ practical $=156$ and practical $\cdot$ difficulty $=96$. Then, assuming $\lambda$ is 2 , the result of the composition is 96 difficulty +156 practical $=\left[\begin{array}{lllll}96 & 1704696 & 1944 & 624\end{array}\right]$. This is now an asymmetric function of $\mathbf{u}$ and $\mathbf{v}$, where $\mathbf{v}$ is stretched by a factor $\lambda$ in the direction of $\mathbf{u}$. However, it is also a more complex type of function, being quadratic in $\mathbf{u}$ (Equation 4.4).

Again, we can think of the composition of $\mathbf{u}$ with $\mathbf{v}$, for this function (Equation 4.21), in terms of a matrix $\mathbf{U}$ which acts on $\mathbf{v}$.

$$
\begin{gathered}
U_{i, j}=(\lambda-1) u_{i} u_{j} \\
U_{i, i}=\left(\sum_{k} u_{k} u_{k}\right)+(\lambda-1) u_{i} u_{i}
\end{gathered}
$$

Where $i, j$ and $k$ range over the dimensions of the vector space. 
The matrix $\mathbf{U}$ has one eigenvalue which is larger by a factor of $\lambda$ than all the other eigenvalues, with the associated eigenvector being $\mathbf{u}$. This corresponds to the fact that the action of this matrix on $\mathbf{v}$ is a dilation which stretches $\mathbf{v}$ differentially in the direction of $\mathbf{u}$. Intuitively, this seems like an appropriate way to try to implement the idea that the action of combining two words can result in specific semantic aspects becoming more salient.

\subsection{Conclusions}

We have proposed a general framework for vector composition based on the assumption that composition is a function of the parts, the syntactic relation between them and some additional background knowledge. Within this framework, we discussed a range of proposals for vector combination functions: from the tensor products of Smolensky (1990) to the predication algorithm of Kintsch (2001), and introduced a number of novel functions.

This chapter has discussed a number of vector composition functions from a purely theoretical perspective. To identify which of these proposals is most appropriate for modelling semantic composition in natural language requires some form of empirical evaluation. In Chapter 5, we will apply these models to the task of predicting subject similarity ratings for pairs of phrases. These experiments will cover a range of structures, examining the dependence on syntax, and will draw on real phrases extracted from a corpus. By correlating the models' predicted similarity ratings to ratings gathered in human experiments, we will demonstrate that certain composition models are more effective than others, and that the success of any particular vector composition function is dependent on the underlying semantic representation. 


\section{Chapter 5}

\section{Modeling Phrase Similarity}

This chapter describes the experiments we ran to evaluate distributional models of composition in terms of their ability to predict similarity ratings for simple phrases. The fundamental semantic information which can be extracted from distributional models is the similarity between pairs of representations and their evaluation is typically made in terms of tasks which rely on this property, for example priming (Lund and Burgess, 1996; Landuaer and Dumais, 1997) or synonymy identification (Bullinaria and Levy, 2007; Landauer and Dumais, 1996). Thus, the ability to predict human similarity judgements for phrases is a natural extension of the word level evaluations. Experiment 1 takes its inspiration from Kintsch (2001), and constructs a set of pairs of sentences consisting of a subject and a verb. After collecting subject similarity ratings for these pairs, we evaluate the models described in the previous chapter in terms of their ability to predict these ratings. Experiment 2 improves on some of the limitations of the materials of Experiment 1 and extends the investigation to adjective-noun, noun-noun and verb-object phrases.

\subsection{Methodology}

Both experiments gather similarity ratings for short phrases and then evaluate the composition models in terms of their correlations with those ratings. We focus on simple 
structures that essentially consist of just two content words. This simplicity allows us to investigate a single syntactic relationship at a time and exclude other confounding factors that might arise in more complex structures. We calculate a predicted similarity for such a pair of phrases by composing the representations of the constituents and then comparing the resulting phrase representations. So, given some composition function, $f(\cdot, \cdot)$, and two phrases $a_{1} b_{1}$ and $a_{2} b_{2}$, we apply $f$ to the vectors $\mathbf{u}_{\mathbf{1}}$ and $\mathbf{v}_{\mathbf{1}}$ representing $a_{1}$ and $b_{1}$, respectively, to produce a composite representation, $\mathbf{p}_{\mathbf{1}}$. Analogously, vectors $\mathbf{u}_{\mathbf{2}}$ and $\mathbf{v}_{\mathbf{2}}$ yield $\mathbf{p}_{2}$ as a representation for $a_{2} b_{2}$. Taking the cosine measure of these two phrase vectors, $\mathbf{p}_{\mathbf{1}}$ and $\mathbf{p}_{\mathbf{2}}$, yields a measure of the similarity for the pair of phrases.

Our experiments assess the performance of the simple additive and multiplicative models (see Equations 4.5 and 4.9, respectively), Kintsch's (Kintsch (2001)) model (Equation 4.6), the tensor product (Equation 4.11) and circular convolution (Equation 4.14). Note that Kintsch's model has two free parameters, the $m$ neighbors most similar to the head, and the $k$ of $m$ neighbors closest to its dependent, which we optimized for each experiment on a held out set. In addition to these models, we also considered two models based on the weighted sum of two values. These were the weighted addition model (Equation 4.7) and the dilation model (Equation 4.21), the parameters of which were also tuned on the same held out sets. Table 5.1 gives the details of these composition functions, expressed in terms of the vector components for each model.

In addition to calculating the similarities predicted by these models, we also gathered a set of human similarity ratings against which the models were evaluated. Chapter 3 evaluated semantic representations of individual words against the WordSim353 (Finkelstein et al., 2002) dataset of similarity ratings for word pairs. In this chapter, models of composition will be evaluated against similarity ratings for phrase pairs. Although similarity ratings are more commonly gathered for pairs of words, experiments on larger structures are nonetheless possible. For example, Lapata and Lascarides 


\begin{tabular}{|ll|}
\hline Model & Function \\
\hline Additive & $p_{i}=u_{i}+v_{i}$ \\
Kintsch & $p_{i}=u_{i}+v_{i}+n_{i}$ \\
Multiplicative & $p_{i}=u_{i} \cdot v_{i}$ \\
Tensor Product & $p_{i, j}=u_{i} \cdot v_{j}$ \\
Circular Convolution & $p_{i}=\sum_{j} u_{j} \cdot v_{i-j}$ \\
Weighted Additive & $p_{i}=\alpha v_{i}+\beta u_{i}$ \\
Dilation & $p_{i}=v_{i} \sum_{j} u_{j} u_{j}+(\lambda-1) u_{i} \sum_{j} u_{j} v_{j}$ \\
Head Only & $p_{i}=v_{i}$ \\
\hline
\end{tabular}

Table 5.1: Composition functions considered in our experiments.

(2003) present an experiment where participants rate whether adjective-noun combinations and their paraphrases have similar meanings, whereas other work (Li et al., 2006) elicits similarity judgments for sentence pairs.

Following previous work (Bullinaria and Levy, 2007; Padó and Lapata, 2007; McDonald, 2000), we then use correlation analysis to examine the relationship between the human ratings and the corresponding model predictions. We interpret the strength of these correlations as indicative of how effective the models are at capturing the semantic similarity of phrases as perceived by the experimental subjects.

We correlate the model similarities directly with the individual subject ratings, and our evaluation is therefore based on the ability of the composition models to predict these raw ratings. To avoid making unnecessary assumptions about the form of the relationship between the ratings and the model predictions, we use a non-parametric correlation measure, Spearman's $\rho$. This measure is based solely on the relative rankings of the ratings, rather than their absolute values, and quantifies the extent to which the orderings of the human ratings and model predictions are in concordance. In comparison, parametric measures of correlation will assume the relationship between the 
raw values comes from a specific family of functions, e.g. linear. Because $\rho$ is based on rankings, we can think of it as quantifying the ability of one variable to be predicted in terms of the other, independently of the particular form of the relationship between them.

\subsection{Experiment 1}

As indicated, Experiment 1 is based on a sentence similarity task initially proposed by Kintsch (2001). In his study, Kintsch investigates how the meaning of an ambiguous verb, for example run, changes in the context of specific noun subjects, for example horse or colour. In particular, the contextual meaning of the verb is established in terms of its relation to other verbs, for example gallop and dissolve, which act as landmarks in the space of meanings. So, the horse ran should be closer to the horse galloped, whereas the colour ran should be closer to the colour dissolved. Kintsch applies this to evaluating his predication algorithm, but uses only a small set of hand picked materials (see Frank et al. 2007 for a criticism of Kintsch's 2001 evaluation standards). Here, we automate the selection of items and gather similarity ratings from a large set of experimental participants.

The basic structure of our experiment is based on pairs of simple sentences consisting of a verb and its noun subject. Participants rated the similarity of these pairs and we then evaluated the computational models of composition in terms of their ability to predict these human ratings. Each item consisted of two simple sentences: both having a subject-verb structure, with one based on such a construction identified in the BNC, and the other having the same noun but with the verb replaced with a synonym taken from WordNet (Fellbaum, 1998). The meaning of the original verb in the context of particular noun subject is thus compared to the WordNet synonym, acting as a landmark as in Kintsch (2001). For each verb there are two such landmarks and the nouns are chosen to maximise the variation of verb meaning between them. 


\subsubsection{Materials and Design}

To construct our materials, we first compiled a list of intransitive verbs from CELEX ${ }^{1}$. From these we identified a subset manifesting limited ambiguity, defined as having between two and eight senses in WordNet (Fellbaum, 1998). All occurrences of these verbs with a subject noun were next extracted from a RASP parsed (Briscoe and Carroll, 2002) version of the BNC. From this set of verbs and nouns we eliminated those which occurred less than fifty times in the BNC, to retain only those words for which reliable vector representations could be built. A pair of landmarks were then chosen for each verb using WordNet (Fellbaum, 1998). Specifically, the landmarks were chosen from different synsets of the verb to be maximally dissimilar and to have a frequency in the BNC greater than fifty. The similarity of the verbs was measured using the Jiang and Conrath (1997) measure ${ }^{2}$, which combines a corpus based approach with an approach based on traversing edges through the WordNet taxonomy.

This process produced a set of candidate items, with the structure subject-verb, subject-landmark. However, at this stage we had no guarantee that the nouns selected did actually induce variations in the verb meaning, nor whether the landmarks we had chosen usefully tracked that variation. In fact, manual inspection of these items suggested that a large proportion of them would not produce a great deal of variation in the assigned similarity ratings. We therefore applied a pretest to a set of candidate items and used the results to further filter the materials for the full experiment.

Our set of candidate materials were constructed from 20 verbs, each paired with 10 nouns, and 2 landmarks, to produce 200 sentence triples of the form subject-verb, subject-landmark1, subject-landmark2. In the pretest, subjects saw the reference sentence containing the original verb alongside the comparisons containing the two landmarks and were asked to choose which landmark sentence was most similar to the reference or neither. Our items were converted into simple sentences (all in past tense)

\footnotetext{
${ }^{1}$ http://www.ru.nl/celex/

${ }^{2}$ We used the implementation provided in the WordNet Similarity package Pedersen et al. (2004).
} 
by adding articles where appropriate. The stimuli were administered to four separate groups; each group saw one set of 50 sentence triples. The pretest was completed by 53 participants.

For each reference verb, the subjects' responses were entered into a contingency table, whose rows corresponded to nouns and columns to each possible answer (i.e., one of the two landmarks). Each cell recorded the number of times our subjects selected the landmark as compatible with the noun or not. We used Fisher's exact test to determine which verbs and nouns showed the greatest variation in landmark preference and items with $p$-values greater than 0.001 were discarded.

This yielded a reduced set of experimental items (120 in total) consisting of 15 reference verbs, each with 4 nouns, and 2 landmarks. The results of the pretest also allowed us to identify which landmark ought to be most similar for each subject-verb sentence. Table 5.2 gives examples of these high and low similarity landmarks. Here, burn is a high similarity landmark (High) for the reference The fire glowed, whereas beam is a low similarity landmark (Low). The opposite is the case for the reference The face glowed. We randomly split the set of materials into two groups to be seen by disjoint sets of participants, such that each group contained one item for each noun associated with a verb. In both groups, two nouns for each verb were paired with high similarity landmarks and two with low similarity landmarks.

\subsubsection{Procedure and Subjects}

The elicitation studies were conducted online using Webexp (Keller et al., 2009), an interactive software package for administering web-based psychological experiments. Subjects took part in a self-paced experimental session that lasted approximately 20 minutes. They accessed the experiment using their web browser, which established an Internet connection to the experimental server running WebExp.

Subjects were given instructions that explained the task and provided examples. They were asked to judge the similarity of phrases using a seven point rating scale 


\begin{tabular}{|llll|}
\hline \multicolumn{1}{|c|}{ Noun } & Reference & High & Low \\
\hline \hline The fire & glowed & burned & beamed \\
The face & glowed & beamed & burned \\
\hline \hline The child & strayed & roamed & digressed \\
The discussion & strayed & digressed & roamed \\
\hline \hline The sales & slumped & declined & slouched \\
The shoulders & slumped & slouched & declined \\
\hline
\end{tabular}

Table 5.2: Example Stimuli from Experiment 1

where a high number indicates higher similarity. To familiarize subjects with the similarity rating task, the experiment consisted of a practice phase (of five items), followed by the experimental phase of 60 items; each containing two sentences, one with the reference verb and one with its landmark. The instructions and materials are listed in Appendices B and C. In both phases, the participants saw one phrase pair at a time and rated its similarity by clicking on one of seven buttons displaying the numbers 1 to 7 . The set of practice and experimental items was presented in random order.

74 unpaid volunteers completed the experiment, recruited by postings to local email lists. 14 participants were eliminated because they were non-native English speakers. This left 60 subjects for analysis. 35 participants were male and 25 female, 55 were right-handed, and 5 left-handed. The subject ages ranged from 15 to 55 , the mean was 28 . Their ratings were randomly split into a development set of 12 participants and a test set of 48 participants.

\subsubsection{Model Parameters}

Our compositional models utilised the distributional representations constructed on the lemmatised BNC described in Chapter 3. The first of these models is a simple semantic space based on word co-occurrence and the other is an LDA model based on 
the document structure of the corpus. Both models define their components in terms of a ratio of probabilities:

$$
v_{i}=\frac{p\left(c_{i} \mid t\right)}{p\left(c_{i}\right)}
$$

and also use the cosine similarity measure:

$$
\cos (\mathbf{u}, \mathbf{v})=\frac{\mathbf{u} \cdot \mathbf{v}}{\sqrt{\mathbf{u} \cdot \mathbf{u}} \sqrt{\mathbf{v} \cdot \mathbf{v}}}
$$

The dimensionalities of the two spaces are 2,000 and 100 for the simple semantic space and the LDA model respectively

The similarity ratings in the development set were used to optimise the parameterised composition models: the weighted addition model, the dilation model and Kintsch's predication model. Kintsch's model contains two free parameters: $m$, the number of neighbours of the predicate which are used in the spreading activation network, and $k$, the number of these neighbours which are eventually summed with the argument. We considered values of 1, 2, 5, 10, 20, 50 and 100 for these parameters and achieved the best results with $m=100$ and $k=1$ for the simple semantic space, and $m=10, k=1$ for the LDA space. The parameters of the weighted addition model define what proportion of head and dependent vectors are summed to produce a representation of their composition. We performed a grid search in steps of $1 \%$, from $0 \%$ to $100 \%$, and found the best results with $71 \%$ of the verb and $29 \%$ of the noun for the simple semantic space whereas for the LDA space the sum was $100 \%$ verb. In the dilation model, the parameter $\lambda$ controls the factor by which one vector is stretched in the direction of the other, and this parameter was optimised in another grid search. For both spaces, the verb was dilated by the noun, with $\lambda=5.56$ for the simple semantic space, and $\lambda=16.67$ for the LDA space.

\subsubsection{Results}

The reliability of the collected judgments is important for our evaluation experiments; we therefore performed several tests to validate the quality of the ratings. First, we 


\begin{tabular}{|c|ccc|}
\hline & Mean & SD & SE \\
\hline High & 5.05 & 1.61 & 0.038 \\
Low & 3.25 & 1.75 & 0.041 \\
\hline
\end{tabular}

Table 5.3: Descriptive statistics for similarity experiments.

examined whether participants gave high ratings to high similarity sentence pairs and low ratings to low similarity ones. Table 5.3 reports means, standard deviations and standard errors of the similarity ratings for items within the two groups. As we can see sentences with high similarity landmarks are perceived as more similar to the reference sentence. A Wilcoxon rank sum test confirmed that the difference is statistically significant $(p<0.01)$. We also measured how well humans agree in their ratings. Inter-subject agreement gives an upper bound for the task and allows us to interpret how well our models are doing in relation to humans. To calculate inter-subject agreement we used leave one-out resampling. The technique is a special case of $n$ fold cross-validation (Weiss and Kulikowski, 1991) and has been previously used for measuring how well humans agree on judging semantic similarity (Resnik and Diab, 2000; Resnik, 1999). For each subject, we calculated the correlation of his ratings with the ratings of the remaining subjects. The average correlation across subjects was $\rho=0.44$. We believe that this level of agreement is satisfactory given that naive subjects are asked to provide judgments on fine-grained semantic distinctions (see Table 5.2).

Table 5.4 shows the correlation of the compositional models with the human ratings. For the LDA models, only composition based on dilation produces a significant $(p<0.01)$ value of $\rho$. The other forms of composition show values of $\rho$ distributed around zero. In contrast, the simple semantic space models appear to be more effective. In this case, all except the simple additive model are significant $(p<0.01)$. The highest correlations are achieved by the simple multiplicative model, the dilation model 


\begin{tabular}{|lcc|}
\hline Model & SSS & LDA \\
\hline Additive & 0.02 & -0.02 \\
Kintsch & 0.15 & -0.02 \\
Multiplicative & 0.18 & 0.02 \\
Tensor Product & 0.07 & -0.03 \\
Convolution & 0.07 & -0.02 \\
Weighted Additive & 0.07 & -0.03 \\
Dilation & 0.16 & 0.05 \\
Head Only & 0.07 & -0.03 \\
Humans & \multicolumn{2}{c}{0.44} \\
\hline
\end{tabular}

Table 5.4: Correlation coefficients of model predictions with subject similarity ratings (Spearman's $\rho$ ) using the simple semantic space (SSS) and latent dirichlet allocation (LDA) models.

and Kintsch's model, which are not significantly different from each other, but are significantly higher than the remaining models. Finally, the tensor product, convolution, weighted additive and head only models all attain very similar levels of correlation, among which only the tensor product and head only models are significantly different $(p<0.01)$.

\subsubsection{Discussion}

The results of this experiment identify the multiplicative composition model on the simple semantic space as the most effective predictor of the subject similarity ratings. In comparison, all the models based on LDA produce much weaker results. One potential explanation for this disparity is the fact that most of the comparisons involve only fine grained differences in meaning: e.g his thoughts strayed - his thoughts roamed. In Chapter 3, we found that LDA's performance was lower on the synonymy identifi- 
cation task, which tests the representations for highly similar items, rather than across the whole spectrum. If LDA is less effective at making discriminations between highly similar items then this would explain the difference between its results and those of the simple semantic space.

However, even the simple semantic space does not achieve particularly high correlations and certainly not correlations comparable to the intersubject comparison $(\rho=$ 0.44). There are at least two issues which might be relevant to explaining these low correlations. The first is the previously discussed issue of the fine-grained discriminations in meaning that the task required. This is a result of the fact that all of the items are based on a pair of sentences in which the verb of one is replaced with a synonym in the other. In many cases, these replacements result in only minor changes in meaning, rather than covering a wide range of variation. The second issue is that many of these replacements result in implausible items. For instance, one of the WordNet synonymns of boom is prosper and this results in an item containing the sentence the noise prospered. Given that it is difficult to interpret what is meant by such a phrase, it is difficult to see how participants could validly assign semantic similarities to such items.

\subsection{Experiment 2}

Experiment 2 overcomes a number of the limitations in the materials used in Experiment 1 . Whereas the latter only investigated subject-verb constructions, the materials we describe in this section cover adjective-noun, noun-noun and verb-object constructions. These new materials also avoid the restriction of having only one word change between the phrases within an item. Experiment 1 generated its comparison sentences by retaining the same noun and replacing the original verb with a synonym, resulting in a limited variation in meaning. Here, the items consist of a pair of entirely distinct phrases both of which are attested in the BNC. Selecting frequent phrases not only avoids the implausible phrases which arose in Experiment 1, it also allows us to test a 
non-compositional model based on treating the phrase as a single linguistic unit with its own distributional properties.

The materials of Experiment 2 also cover a much wider distribution of semantic similarity, from practically wholly unrelated to almost entirely synonymous. Evaluating on such materials is important in terms of showing that our models can handle a set of items representative of the full range of variation. However, simply making gross discriminations between highly similar items and highly dissimilar items is likely to be too easy, and so fail to differentiate the performance of the models. Equally, evaluating on extremely fine grained distinctions in similarity is likely to be too difficult and also fail to differentiate the models successfully. Therefore, our materials are constructed in three distinct processes, designed to produce high, medium and low similarity items respectively. The alternative of simply randomly sampling items from the set of all possible phrase pairs, while unbiased, yields in the main largely unrelated pairs, with very few items of even medium similarity. In contrast, the high medium and low similarity "bands" contain a wide range of semantic similarities and overlap substantially with each other, resulting in an effective evaluation across the whole spectrum of similarity.

A further concern was that effects due to the vocabulary of the items should be controlled for. In other words, we wanted to avoid the possibility that differences in the performance of the models or in the participant ratings could be attributed to differences in the vocabulary of the similarity bands. To this end, the items within each band consisted of the same set of phrases combined in different pairings to produce the desired range of similarities for each band. Participants were randomly divided into three groups, with each participant seeing each phrase only once, and each phrase being part of a high, medium and low similarity pairing across the three groups. 


\subsubsection{Materials and Design}

Initially, we extracted all adjective-noun, noun-noun, and verb-object combinations attested in the BNC, parsed with RASP (Briscoe and Carroll, 2002). Our high similarity items were compiled from phrases occurring at least 100 times in the BNC. For each grammatical construction, any two phrases were considered highly similar if swapping their heads resulted into two new phrases which were also attested in the BNC at least 100 times. For example, practical difficulty-economic problem is a candidate high similarity item, because practical problem and economic difficulty are also high frequency phrases. Our hypothesis was that the phrases resulting from this recombination process must exhibit some semantic overlap, especially if they appear often in the BNC. This procedure resulted in 11,476 candidate adjective-noun, 366 noun-noun, and 1,004 verb-object pairs. For subject-verb constructions, only 36 pairs were produced, which was too few to construct a full set of items from.

In order to reduce the set of items to a more manageable size and more importantly to guarantee that the phrases were indeed semantically similar, we resorted to WordNet (Fellbaum, 1998). We used a well-known dictionary-based similarity measure, originally proposed by Lesk (1986), to rank the candidate phrase pairs. According to this measure, the semantic relatedness of two words is proportional to the extent of overlap of their dictionary definitions ${ }^{3}$ (glosses in WordNet). We computed the similarity of two phrases, as the sum of the similarities of their constituents. The 36 highest ranking phrase pairs (for each grammatical structure) on this measure formed our highsimilarity items (e.g., vast amount-large quantity, telephone number-phone call, start work-begin career). These 36 phrase pairs (72 phrases in total) were subsequently recombined to produce the items in the medium and low similarity bands. This was done in order to eliminate any confounding effects relating to the vocabulary of the individual phrases. By choosing the same same set of phrases to construct all three bands, differences between bands cannot be attributed to lexical choice but instead to

\footnotetext{
${ }^{3}$ We used the implementation provided in the WordNet Similarity package Pedersen et al. (2004).
} 
their actual similarity relations.

Specifically, the high similarity phrases were first randomly split into three groups, and then candidate items for the remaining bands were constructed by pairing phrases from each of these groups. So, each phrase was used three times in our materials: once in a high similarity pair, once in a medium pair and once in a low pair. For example, practical difficulty from the first group was paired with effective way from the third group to produce the item practical difficulty-effective way. The Lesk similarity for each of these pairs was calculated as above and the 36 highest ranking items on this measure were selected, subject to the constraint that each phrase was only used once in each group. This produced a set of Medium similarity items, which, while they scored reasonably highly on the WordNet-based measure, did not have the recombination property described above (e.g., social activity-economic condition, market leader-board member, discuss issue-present problem). A further 36 items were selected from the same set of candidate items, though in this case by choosing the lowest ranking items. This produced a set of Low similarity items (e.g., practical difficultycold air, phone call-state benefit, drink water-use test). The entire list of experimental stimuli is given in Appendix E.

Thus, in our experimental design, the subject ratings and model predictions were the dependent variables, and the bands and groups acted as blocking factors with a $3 \times 3$ structure. For each phrase type (i.e., adjective-noun, noun-noun, and verb-object) we collected 108 items, 12 for each band by group cell. The selected verb-object pairs were converted into a simple sentence by adding a subject and articles or pronouns where appropriate. All verbs were in the past tense. The sentential subjects were familiar proper names (BNC corpus frequency $>30$ per million) balanced for gender.

\subsubsection{Procedure and Subjects}

The experimental procedure followed a very similar course to that of Experiment 1 . Subjects took part in a self-paced experimental session that lasted approximately 20 
minutes, using Webexp (Keller et al., 2009). This began with instructions that explained the task and provided examples (see Appendix D). The experiment itself consisted of a practice phase (of five items), followed by the experimental phase. In both phases, the participants saw one phrase pair at a time and rated its similarity by clicking on one of seven buttons displaying the numbers 1 to 7 . The set of practice and experimental items was presented in random order.

The experiment was completed by unpaid volunteers, all self-reported native speakers of English, recruited by postings to local email lists. The adjective-noun experiment was completed by 88 participants; 69 subjects took part in the noun-noun experiment and 91 in the verb-object experiment. 14 participants were eliminated because they were non-native English speakers. The data of 30 subjects was excluded after inspection of their responses revealed anomalies in their ratings. For example, they were pressing buttons randomly, alternately, or rated all phrase pairs uniformly. This left 204 subjects for analysis, 72 for the adjective-noun, 56 for the noun-noun, and 76 for the verb-object experiment. 35 participants were male and 73 female, 94 were right-handed, and 14 left-handed. The subject ages ranged from 17 to 66, the mean was 31. Participants were randomly allocated to a development set, used for optimizing model parameters, and a test set on which the final evaluation of all models was carried out. For each experiment the test set contained 36 participants, and the development set contained 18 .

\subsubsection{Model Parameters}

The same distributional representations were used as in Experiment 1 Section 5.2.3 and parameters were also tuned on the development set as before. The final parameters for Kintsch's model and our weighted addition and dilation models are shown in Tables 5.5, 5.6 and 5.7, respectively.

Additionally, as a baseline, we considered a non-compositional model which treats the phrase as a single target unit and thus extracts a vector representation for the whole 


\begin{tabular}{|l|cc|cc|}
\hline & \multicolumn{2}{|c|}{ Semantic Space } & \multicolumn{2}{|c|}{ LDA } \\
& $m$ & $k$ & $m$ & $k$ \\
\hline Adjective-Noun & 10 & 10 & 50 & 10 \\
Noun-Noun & 100 & 1 & 50 & 1 \\
Verb-Object & 50 & 10 & 100 & 1 \\
\hline
\end{tabular}

Table 5.5: Parameters for Kintsch's composition model.

\begin{tabular}{|l|cc|cc|}
\hline & \multicolumn{2}{|c|}{ Semantic Space } & \multicolumn{2}{|c|}{ LDA } \\
& $\alpha$ & $\beta$ & $\alpha$ & $\beta$ \\
\hline Adjective-Noun & 0.88 & 0.12 & 0.65 & 0.35 \\
Noun-Noun & 0.32 & 0.68 & 0.34 & 0.66 \\
Verb-Object & 0.31 & 0.69 & 0.50 & 0.50 \\
\hline
\end{tabular}

Table 5.6: Parameters for weighted addition composition models.

phrase. This baseline is only applicable to the standard semantic space. LDA derives semantic representations for individual words rather than word combinations ${ }^{4}$.

\subsubsection{Results}

As before, we performed an initial analysis to confirm that our experiment had produced reliable ratings across a range of similarities. We first performed a series of Kruskal-Wallis rank sum tests to examine the relationship between our similarity bands and the elicited similarity ratings. Within each experiment, the subject ratings were significantly different $(p<0.01)$ across all bands, and also between each pair of bands.

\footnotetext{
${ }^{4}$ It would be possible to train an LDA model which contained representations for such word pairs. We would simply treat each pair as a single unit as before and enter counts for these units into the standard word document matrix. However, this would result in a new model, distinct from the one evaluated in Chapter 3. In particular, vectors from one representation would not be comparable to those of the other. In contrast, for the simple semantic space the single word and word pair representations are essentially in the same space and are directly comparable.
} 


\begin{tabular}{|l|rr|rr|}
\hline & \multicolumn{2}{|c|}{ Semantic Space } & \multicolumn{2}{|c|}{ LDA } \\
& $\lambda$ & Direction & $\lambda$ & Direction \\
\hline Adjective-Noun & 16.7 & Adjective & 2.2 & Noun \\
Noun-Noun & 8.3 & Head Noun & 7.1 & Head Noun \\
Verb-Object & 7.7 & Verb & 6.3 & Verb \\
\hline
\end{tabular}

Table 5.7: Parameters for dilation models.

\begin{tabular}{|l|ccc|ccc|ccc|}
\hline & \multicolumn{3}{|c|}{ Adjective-Noun } & \multicolumn{3}{c|}{ Noun-Noun } & \multicolumn{3}{c|}{ Verb-Object } \\
\hline \multirow{4}{*}{ High } & Mean & SD & SE & Mean & SD & SE & Mean & SD & SE \\
Medium & 3.76 & 1.926 & 0.093 & 4.13 & 1.761 & 0.085 & 3.91 & 2.031 & 0.098 \\
Low & 2.50 & 1.814 & 0.087 & 3.04 & 1.732 & 0.083 & 2.85 & 1.775 & 0.085 \\
& 1.99 & 1.353 & 0.065 & 2.80 & 1.529 & 0.074 & 2.38 & 1.525 & 0.073 \\
\hline
\end{tabular}

Table 5.8: Descriptive statistics for similarity experiments (adjective-noun, noun-noun, and verb-object), by subjects.

Furthermore, the statistics in Table 5.8 demonstrate that the mean ratings show the correct ordering (High $>$ Medium $>$ Low) and that there is substantial overlap between each band. These results confirm that our procedure for generating the materials produced items with a wide range of similarities.

We further examined how well the participants agreed in their similarity judgments for adjective-noun, noun-noun, and verb-object combinations. As in Experiment 1, we quantified this by correlating each subjects ratings with the remaining subjects and averaging over subjects. For the adjective-noun experiment, the average of Spearman's $\rho$ was $.52(\operatorname{Min}=0.35, \operatorname{Max}=0.73, \mathrm{SD}=0.12)$, for the noun-noun experiment .49 $(\operatorname{Min}=0.36, \operatorname{Max}=0.58, \mathrm{SD}=0.06)$, and for the verb-object experiment $0.55(\mathrm{Min}$ $=0.45, \mathrm{Max}=0.65, \mathrm{SD}=0.06)$. These results indicate that the participants found the similarity rating task relatively difficult, though still produced ratings with a reasonable 


\begin{tabular}{|lccc|}
\hline Model & Adjective-Noun & Noun-Noun & Verb-Object \\
\hline Additive & 0.36 & 0.39 & 0.30 \\
Kintsch & 0.32 & 0.22 & 0.29 \\
Mutliplicative & 0.46 & 0.49 & 0.37 \\
Tensor Product & 0.41 & 0.36 & 0.33 \\
Convolution & 0.09 & 0.05 & 0.10 \\
Weighted Additive & 0.44 & 0.41 & 0.34 \\
Dilation & 0.44 & 0.41 & 0.38 \\
Target Unit & 0.43 & 0.34 & 0.29 \\
Head Only & 0.43 & 0.17 & 0.24 \\
Humans & 0.52 & 0.49 & 0.55 \\
\hline
\end{tabular}

Table 5.9: Correlation coefficients of model predictions with subject similarity ratings (Spearman's $\rho$ ) using a simple semantic space.

level of consistency.

Table 5.9 shows the correlation of the subjects' similarity ratings with the models' predictions when using a simple co-occurrence-based semantic space. All models are significantly correlated with the human judgments ( $p<0.01)$, except circular convolution when applied to noun-noun combinations. Let us first consider, the simpler composition models based on vector addition (see Additive and Kintsch in the table). Within this class of models we observe that Kintsch's model fails to improve on the simple additive model and is significantly ${ }^{5}$ worse $(p<0.01)$ than the standard additive model for the noun compounds.

Within the class of multiplicative models (see Multiplicative, Tensor Product, and Circular Convolution in Table 5.9), the simple multiplicative model significantly $(p<0.01)$ outperforms all other models. Specifically, both tensor products and circular convolu-

\footnotetext{
${ }^{5}$ We examined whether the correlations achieved differ significantly using a $t$-test (Cohen and Cohen, 1983).
} 
tion are significantly worse $(p<0.01)$. The multiplicative model is also significantly better than the Additive one $(p<0.01)$. These results are observed across the board, with adjective-noun, noun-noun, and verb-object combinations. It is worth noting that circular convolution is the worst performing model. The tensor product itself, from which circular convolution is derived, is significantly better $(p<0.01)$ in all experiments. This indicates that the manner in which circular convolution projects the tensor product down onto a lower dimensional space does not preserve the useful information the product may have contained. In addition, the fact that the tensor product is significantly worse than the simple multiplicative model indicates that the off diagonal elements of the product, which are discarded in the simple multiplicative model, are probably not contributing much to the composition.

We next consider the Weighted Additive and Dilation models. Recall that these models are parametrized; in dilation models one word dilates the other by a factor $\lambda$ whereas the weighted additive model weights the constituents in the summation differentially. As shown in Table 5.9 the two models perform similarly. This is not entirely surprising, as both consist of a sum of the constituents multiplied by scalar factors (see Equations 4.7 and 4.21). The performance of these models does not differ significantly, except in the case of verb-object combinations where the dilation model performs significantly better $(p<0.01)$. This model also fares similarly to the multiplicative model. The two models yield correlations that are not significantly different, except in the case of noun-noun combinations, where the multiplicative model is better $(p<0.01)$.

The two non-compositional models, Target Unit and Head Only, perform worse than multiplicative composition, with this difference reaching significance $(p<0.01)$ for noun-noun and verb-object combinations. In general, the target unit model performs better than the head only model (it obtains significantly $(p<0.01)$ better correlations for noun-noun combinations). This is not surprising, the target unit model may be non-compositional, but nevertheless represents the semantics of the two words 


\begin{tabular}{|lccc|}
\hline Model & Adjective-Noun & Noun-Noun & Verb-Object \\
\hline Additive & 0.37 & 0.45 & 0.40 \\
Kintsch & 0.30 & 0.28 & 0.33 \\
Multiplicative & 0.25 & 0.45 & 0.34 \\
Tensor Product & 0.39 & 0.43 & 0.33 \\
Convolution & 0.15 & 0.17 & 0.12 \\
Weighted Additive & 0.38 & 0.46 & 0.40 \\
Dilation & 0.38 & 0.45 & 0.41 \\
Head Only & 0.35 & 0.27 & 0.17 \\
Humans & 0.52 & 0.49 & 0.55 \\
\hline
\end{tabular}

Table 5.10: Correlation coefficients of model predictions with subject similarity ratings (Spearman's $\rho$ ) using the LDA topic model.

participating in the composition more faithfully, whereas the head only model offers a more impoverished representation as it is based solely on the meaning of the head.

In sum, we find that the multiplicative, weighted additive and dilation models perform overall best. The multiplicative model has a slight advantage as it has no parameters (other than the semantic space representing the individual words), and is conceptually simpler than the other two models. On the down side, it is does not take syntactic information into account, whereas the other two can modulate the role of syntactic structure by tuning the appropriate weights. We should also note that in all cases our compositional models fall behind the human upper bound (see the last row in Table 5.9). The multiplicative model comes close when applied to noun-noun combinations.

We now turn our attention to the compositional models which employ the LDA topic model. As can be seen in Table 5.10, Kintsch's model remains worse than the simple additive model for all constructions considered here (and the differences are 
statistically significant $(p<0.01))$. Regarding compositional models based on multiplication, we observe that tensor products and the simple multiplicative model yield comparable performances for noun-noun and verb-object combinations. They differ for adjective-nouns with the tensor product being significantly better $(p<0.01)$. Circular convolution remains the worst performing model. Not surprisingly, Weighted Additive and Dilation models obtain almost identical performances. And they are not significantly different from the simple Additive model. The non-compositional model (Head Only) is significantly worse than these models. Comparing the spatial and topicbased representations reveals that the multiplicative composition model on the simple semantic space is significantly $(p<0.01)$ better than the dilation model with LDA, except in the verb-object experiment, where there is no significant difference between them.

\subsubsection{Discussion}

For the simple semantic space, no model significantly outperformed the simple multiplicative model. In contrast, for the LDA representations, no model significantly outperformed the simple additive model. The weaker performance of the multiplicative model on the LDA representations can be attributed to the fact that these vectors are much sparser, and multiplication of their components tends to result in a loss of useful information when a non-zero component is multiplied by zero. More difficult to explain is the fact that no syntax based model outperforms either of these simple bag-of-words models. In Section 2.3 we discussed Pinker's (1994) assertion that composition is not blending. Yet, the simple additive and multiplicative models are essentially forms of blending, producing composed vectors which are somewhat similar to both the inputs. Syntactic models, which differentiate the contributions of the two constituents, seem like they ought to provide a more effective model of semantic composition. For example, our novel weighted additive and dilation models treat their constituents differently and also allow different parameter values for each syn- 
tactic structure. However, while they produced competitive results on all the phrase types for both spaces, they did not significantly outperform the syntaxless rival. This may be attributable to the fact that while they do allow a dependence on syntax, it is not a particularly sophisticated dependence. In fact, both models are essentially the weighted sum of the two constituents, and thus the composed vector is something like both inputs, but a little more like one than the other. Such a simple treatment of syntax appears to be insufficient to outperform the simpler non-syntactic models. Nonetheless, these models did outperform the other syntax based alternatives, in particular circular convolution.

Circular convolution is designed to allow two vectors to be bound into a single representation of the same dimension, in such a way that this composite vector can be broken down into the original components again. As we discussed in Section 2.2, this means that the representations it forms act like memories for complex structures, allowing the recall of component parts after binding. However, we then argued in Section 2.3 that experiments on sentence recall (Sachs, 1967, 1988; Begg, 1971) demonstrate that semantic composition is not simply equivalent to memory for constituents and their stucture. Our experiments confirm this line of reasoning: the poor performance of circular convolution indicates that vector binding is not by itself sufficient to model the semantic processes of composition. While circular convolution may be an adequate model of symbol concatenation, this syntactic operation is not the same thing as semantic composition. In modeling the composition of a predicate and argument, it is not enough to simply bind the representation of one to the representation of the other, we must instead model the interaction between their meanings and their integration to form a whole.

Interestingly, circumventing this issue, by treating the phrase as a single noncompositional unit, yields results inferior to several of the compositional models. This is somewhat surprising, as the target unit approach might be expected to produce a representation more specific to the meanings of the words as they occur in the phrase. 
In contrast, the semantic representations used in the compositional approach are general to all instances of the constituent words. Nonetheless, the compositional models produce representations that are more effective in predicting human similarity judgements.

Currently, few of our models outperform the simple multiplicative model on this task, even though it lacks a dependence on syntax. Of course, this simplicity has advantages: it is fast and easy to compute, it does not need to be optimised for each syntactic structure, and it can be applied in a bag-of-words manner, without regard to syntactic structure. This latter trait will be exploited in the following chapter to construct incremental representations for language modelling. Nonetheless, a model of composition which can exploit syntax effectively is still desirable. However, while our models lack the necessary syntactic sophistication the subjects ratings gathered in this chapter will continue to provide a means of evaluation as distributional models of composition develop.

\subsection{Conclusions}

In this chapter we demonstrated that distributional models of semantic composition can be evaluated in terms of their ability to predict human similarity ratings for pairs of phrases. We gathered such judgements for subject-verb, adjective-noun, noun-noun and verb-object constructions, and used them to evaluate a number of composition functions applied to two distinct forms of distributional representation: a simple semantic space and an LDA topic based model. The correlation of the human ratings with the model predictions reveals that, of the unparameterised models, the simple multiplicative model is the best performing model on the simple semantic space whereas the simple additive model is best for LDA. We attribute the disparity in performance to the sparsity of the LDA representations. The simple semantic space contains highly distributed representations, with the semantic content spread across the great variety of 
contexts a target word occurs in. In contrast, topic models tend to produce representations in which the vast majority of topics are inactive (i.e., zero) and when these topics are multiplied by other topics, the result is zero. Thus, multiplicative combinations of sparse representations tend to result in a loss of useful information. Among the parameterised models, the dilation model gives results close to the best performance across both spaces.

The major limitation of these evaluations is that they are constrained to very simple constructions. As argued in Section 5.1, this was a deliberate choice allowing us to isolate a single syntactic structure and eliminate other confounding factors. Nonetheless, future work will need to evaluate the representations of more complex structures, involving not only multiple syntactic relationships but also more complex constructions such as relative clauses.

However, before tackling this phrase similarity task in more depth, we would like to confirm that the results presented in this chapter are relevant outside the domain of the experiments we have considered so far. In particular, finding a similar pattern of results in other tasks would help to underscore the validity of this evaluation. Moreover, it would be desirable to be able to automate the evaluation of compositional models on a corpus, without requiring human ratings. A programme of human experiments to cover the full richness and diversity of language would be impractical. Ideally, a corpus based task would also demonstrate the practical utility of distributional models of composition.

In the following chapter, we will adapt the models of phrase similarity used here to produce a compositional language model. From the assumption that upcoming words should be semantically coherent with their history, we will derive probabilities from distributional representations of words and histories. In particular, the representation of prior history will be constructed incrementally using a compositional model. We will evaluate the effectiveness of these composition operations in terms of the perplexity of the resulting language model on a test set. 


\section{Chapter 6}

\section{Language models based on Vector}

\section{Composition}

Chapter 5 evaluated a number of vector composition functions against similarity ratings for pairs of short phrases. While this approach does give a fairly direct insight into semantic similarity and compositional representations, it can be criticised on a number of fronts. Firstly, the amount of data that can be gathered in this way is limited by the need to recruit participants and convince them to repetitively perform the task without losing interest. Secondly, the materials on which the judgements are made are limited and have been carefully selected, rather being representative of the range of constructions found in natural text. Lastly, the elicited ratings are the product of an artificial experimental task, the relation of which to natural linguistic behaviour is unclear.

Ideally, we would like to augment our evaluation with a task which is based on large quantities of natural data. In addition we would like to demonstrate that the issue of vector composition has practical consequences. For these reasons, the present chapter investigates how vector composition models from the previous chapters can be applied to language modelling. Language modelling is one of the fundamental components of computational linguistics, being applied in speech recognition, machine translation and many other tasks. For our purposes, it also provides a means of testing whether 
the semantic relationships hypothesised by our compositional models relate to what is found in naturally occurring linguistic data. The basic idea, which has been implemented in a variety of ways (Bellegarda, 2000; Coccaro and Jurafsky, 1998; Gildea and Hofmann, 1999), is that each new word in a sentence should be semantically similar to the prior context. This simple approach to modelling the semantic dependencies between the parts of a sentence allows us to enforce an overall semantic coherence on its subject matter.

The contribution of the work presented here is that we examine the question of how to construct a representation of prior context by composing the vectors representing the words it contains. This requires an incremental approach to the construction of semantic representations, and the simple additive and multiplicative functions are well suited to this task. We show that these composition models can function as a language model, with relatively minor modifications. We evaluate them for both the simple semantic space and LDA vectors in terms of the perplexity of the derived language model on a held out set.

As a stand alone language model, we find that this approach is only weakly predictive, as might be expected given that it has little to say about word order. We therefore use it to modulate the output from a trigram language model, and show that this improves performance by incorporating dependencies to content words outside the trigram window. We also show that interpolating with a syntactic language model results in further improvements.

In Section 6.1, we give an overview of language modelling. We then examine vector composition within this perspective in Section 6.2. Finally, Section 6.3 evaluates the compositional language models and Section 6.4 summarises and draws conclusions from this work. 


\subsection{Language models}

A language model is a means of assigning probabilities to texts, that is to sequences of words. In the research presented here we focus on the probability of sentences, but longer texts, e.g. paragraphs or whole documents, can also be modelled. Such probability distributions have many practical applications for example in guiding speech recognition or machine translation, but they are also relevant to the investigation of the cognitive processes that enable linguistic behaviours. Ultimately, a successful cognitive model of the processes of language production ought to be able to tell us which word sequences are likely to be found in natural text and which are not. Furthermore, the probability of a text can also be used in modelling its comprehension, with high probability sequences being read more quickly than those with low probability.

Thus, there are a number of practical and theoretical reasons for taking an interest in modelling the probabilities of word sequences. At its most general this requires deriving a joint probability model of the sequence. Commonly, however, the joint probability is expressed as a product of conditional probabilities as in Equation 6.1.

$$
p\left(w_{1}, w_{2}, \ldots w_{n}\right)=p\left(w_{1}\right) p\left(w_{2} \mid w_{1}\right) \ldots p\left(w_{n} \mid w_{n-1} \ldots w_{2}, w_{1}\right)
$$

Although this is provably true for all distributions, expressing the joint distribution in this way is most useful when the dependencies being exploited by the language model are indeed of this form, that is when a word is only dependent on previous words to its left. Such a model will be incremental in the sense that it processes the input one word at a time, conditioning the probability of the next word only on the previous history of already processed words. This incrementality can be useful practically, in integrating with the other components of a speech recognition system, and also cognitively consistent with the incrementality of the human language processor (Tanenhaus et al., 1995; Marslen-Wilson, 1973; Konieczny, 2000).

As already intimated, in assigning probabilities to sequences, language models exploit dependencies between the parts. So, for example either is typically followed at 
some point by or, and a language model, sensitive to such long range dependencies, may use this fact to deflate the probabilities of sequences which break this rule. Alternatively, a language model may be attuned to short range dependencies, such as the fact that chief operating is almost always followed immediately by officer. It is clear that the dependencies within any sentence of a reasonable length will be both numerous and complex, posing difficulties for any system that attempts to handle all of them. The ideal language model would of course exploit all the structure of language to make the most accurate predictions of the probability of a text, but in practice the need to estimate the model's parameters from a corpus makes this impractical for current approaches. Instead, effective language models exploit a limited set of the most important dependencies. In the following discussion we outline the various types of dependencies and how they may be modelled.

\subsubsection{Syntactic Models}

The obvious way to characterise linguistic structure would be in terms of syntax, and syntactic language models express the probability of sentences in terms of these dependencies. For example a Probabilistic Context Free Grammar (PCFG) associates a probability with each rule in a CFG (Booth, 1969). The joint probability of a parse tree, $T$, is then the product of the rules, $r$, that expand each node, $n$, and the probability of a sentence, $S$, is a sum over the set of its parse trees, $\tau(S)$.

$$
p(S)=\sum_{T \in \tau(S)} p(T)=\sum_{T \in \tau(S)} \prod_{n \in T} p(r(n))
$$

However, the probabilities produced by a PCFG are not very predictive. While this approach captures dependencies at the level of syntactic types, e.g. nouns are modified by adjectives, it tends to ignore lexical dependencies, e.g the adjective happy rarely modifies the noun sky.

To remedy this shortcoming, the grammar can be lexicalised. That is, non-terminals are annotated with some information about the lexical items into which they expand. 
For example, Charniak (2001) implements a language model based on immediate head parsing. In this case the expansion of a constituent is conditioned on its lexical head, and this allows the relation between the head and its dependents to be modelled. It does not, however, model relations between dependents of the same head, treating them instead as independent. Of course, additional lexical conditioning information can be introduced into the grammar, but dividing the grammatical categories more and more finely will eventually lead to problems with the sparsity of data.

It is also worth noting that conditioning the probability of words on their heads is not generally consistent with incrementality. When a dependent can precede its head, e.g., adjectives precede their head noun, the head will not be available for conditioning the probability of the dependent if the sentence is processed incrementally in a left to right fashion. Roark (2001) develops an incremental syntactic language model by employing a couple of variations. First, a left corner transform is applied to the grammar, which allows complete rooted trees to be built on the left context by removing the problem of non-terminating left recursion. This allows the identification of all the dependencies in this prior history which could be used to condition the probabilities of upcoming words. Second, a general approach to selecting this conditioning information based on a tree walking algorithm is employed. In practice, the best performing model selects lexical heads where available, and nearby lexical items otherwise, but alternative models also consider non-lexical information, such as POS tags.

Both these language models are supervised, in that they require a treebank of parsed text on which to be trained. Parsing and annotating a corpus by hand is time consuming, and consequently the quantity of such data is much more limited than simple raw text. In practice, syntactic language models are often interpolated with $n$-gram models, which are trained on raw text, to ameliorate this problem. Unsupervised approaches can infer syntactic structures from raw text, but are slower and less accurate. In addition, however a probabilistic grammar is trained, applying it as a language model will be based on a search over the space of parse trees, which is typically resource inten- 
sive. Thus, although syntax gives us the most complete formalism for describing the structure of sentences, in practice the fact that this structure is not directly observable in raw text inhibits its utility.

\subsubsection{Ngram Models}

An alternative to exploiting syntactic structure for language modelling is simply to use linear order. The linear order of words in a sentence is explicit and relatively simple, in contrast to syntax, which has a hierarchical structure and can only be inferred implicitly. In terms of linear order, the strongest dependencies are highly local, between words that are very close to each other. Thus, effective language models can be built by simply conditioning the probabilities on the most recent prior words. In other words, we truncate the history when we consider the conditional probability of a word. These $n$-gram models approximate the full conditional probability of a word by its conditional probability given only the last $n-1$ words, for some low value of $n$, e.g., $n=3$.

$$
p\left(w_{n} \mid w_{n-1}, \ldots w_{2}, w_{1}\right) \approx p\left(w_{n} \mid w_{n-1}, w_{n-2}\right)
$$

Ignoring dependencies to words outside this $n$ word window reduces the complexity of the model to a manageable scale and the probabilities can be derived from counting $n$-grams in a corpus. The simplest approach would be to convert counts directly to probabilities using the maximum likelihood estimate as in Equation 6.4.

$$
p\left(w_{3} \mid w_{2}, w_{1}\right)=\frac{\operatorname{freq}\left(w_{3}, w_{2}, w_{1}\right)}{\text { freq }\left(w_{2}, w_{1}\right)}
$$

Unfortunately, this approach assigns all the probability mass only to $n$-grams which were seen in the training data. However large the training corpus it is unlikely that it will contain every plausible $n$-gram. This means that in applying the model to novel data there will be word sequences which are assigned zero probability. Worse, there will even be some sequences which cannot be assigned a meaningful probability because the denominator in Equation 6.4 is zero. The solution is to use a smoothing 
technique to shift some of the probability mass onto unseen events. Two questions need to be addressed: how much probability mass to reassign and how to distribute it among the unseen events.

There are a number of approaches to answering both these questions. For example, absolute discounting (Ney et al., 1994) provides a very simple means of deciding how much probability mass to re-assign, by simply subtracting the same amount from all non-zero counts. These quantities can then be redistributed to the unseen events. Whereas this approach discounts all counts by the same amount, the Good-Turing (Good, 1953) method takes into account the number of different $n$-grams having a given count in determining the discounts. However discounting is carried out, this still leaves open the question of how to assign it across the zero counts. A common method for distributing the discounted probability mass over unseen events is in terms of back off probabilities (Katz, 1987). The basic idea is to use a lower order model, i.e. with coarser dependencies, to assign probabilities to the zero count events. So, a trigram model could back-off to a bigram model, with the bigram counts being less affected by sparsity, and the bigram model might itself back off to a unigram model. Typically, these lower order models are estimated in the same way as the top level model, in terms of counting $n$-grams. Kneser and Ney (1995) show that this may not be optimal and propose a form of back off in which the lower order models are specifically adapted to the task of making predictions for unseen events.

Another issue that arises in applying a trained model to a novel corpus is that of domain differences. It is obvious that the vocabulary of documents from a news corpus will differ significantly from those in a biomedical corpus, and a model trained on one will not be optimal when tested on the other. Moreover, even within the same general domain, data from different sources can have significantly different statistics. For example, Rosenfeld (1996) found that a trigram model trained on the Wall Street Journal doubled its perplexity when applied to Associated Press articles.

A variety of approaches to domain adaptation for $n$-gram models have been pro- 
posed (e.g., see Bellegarda, 2004). For example, Kuhn and de Mori (1990) proposed interpolating the unadapted model with a model derived from a cache of recently observed words from the test corpus. In this way the probabilities of common vocabulary items from the test domain are increased. While this only incorporates a dependency between a word and its own re-occurence later in the corpus, Rosenfeld (1996) used a maximum entropy framework to select arbitrary trigger word pairs. In this way encountering a given trigger can modulate the probabilities of a number of related words in its wake.

Rather than thinking of these models as adapting a language model to a new domain, we can instead think of them as extending the model to include history outside the $n$-gram window. Both the cache and the triggers allow longer range dependencies between words to be captured. Nonetheless, these models are still based on dependencies between specific individual words, albeit over greater spans.

\subsubsection{Semantic Models}

Both the syntactic and $n$-gram models exploit lexical dependencies to make predictions. But there are of course more general factors which might help in predicting which words are likely to come next. In particular, any given text will have some overall subject matter, and its particular content will generally be coherent and relevant to that subject. A common approach to capturing these sort of effects in a language model is to base the probability of an upcoming word on its similarity to the history of previous words (Bellegarda, 2000; Coccaro and Jurafsky, 1998; Wandmacher and Antoine, 2007; Gildea and Hofmann, 1999). In this way, the language model prefers words which fit the semantic topic established so far. Two issues need to be resolved in implementing this idea. First, a probabilistic measure for comparing the semantic representations of a word and its history is required. Second, building a representation of the history requires some means of combining word representations.

With representations derived from Latent Semantic Analysis (Landuaer and Du- 
mais, 1997) the obvious way to compare vectors is in terms of the cosine measure, and this is the approach taken by Bellegarda (2000) and Coccaro and Jurafsky (1998). Unfortunately, the cosine measure does not directly yield a valid probability because its values do not sum to one. Worse, it even produces negative values for some comparisons. The solution proposed by Coccaro and Jurafsky (1998) is to rescale and normalise these values to produce a valid probability measure. They first add or subtract the same amount to all values, shifting the minimum to zero, and then normalise, producing a distribution that sums to one. Following that, to increase the dynamic range, the values are raised to some power (e.g. 7) and renormalised.

While this method defines the transformation of cosine values into probability estimates algebraically, Bellegarda (2000) takes a different approach by defining this function empirically. Specifically, the probability associated with a given cosine value is based on the number of times a value of that size was encountered in the training data.

Whatever transformation is used, the cosine measure is still nonetheless an imperfect basis from which to derive probabilities. In particular, it is simply a measure of similarity and takes no account of the underlying frequency of the items involved. So, for example, otiose and lazy may be equally similar to some context, but the latter, being more common, may nonetheless be more probable. The approach of Gildea and Hofmann (1999) overcomes this difficulty by using representations constructed with pLSA, which have a direct probabilistic interpretation. The pLSA model is based on the assumption that each document in a collection is a mixture of topics, which are themselves unigram distributions over the vocabulary. The probability of finding a word, $w$, in a document, $d$, is then expressed as a sum over topics, $t$.

$$
p(w \mid d)=\sum_{t} p(w \mid t) p(t \mid d)
$$

If the history can be treated as a kind of document then this expression provides a means of deriving probabilities naturally and directly, avoiding the need for ad-hoc transformations. 
This still leaves the question of how to construct the representation of history. This will involve combining the individual word vectors in some way to form a single representation of the whole prior context. Coccaro and Jurafsky (1998) do this by simply summing them, which is motivated in terms of the geometric interpretation of vectors. Bellegarda (2000) instead appeals to the mathematical foundations of the LSA procedure to represent the history as a pseudo-document, which is basically a weighted sum of the same word vectors. Similarly, Gildea and Hofmann (1999) derive their approach from the representation of documents within pLSA. They use a single iteration of an online EM algorithm to infer a pseudo-document representation for the history.

While these methods of constructing history representations are reasonably well motivated in terms of the assumptions of the models to which they are applied, without an empirical investigation of the performance of a range of approaches we cannot determine how optimal they are. In particular, Chapter 4 discussed a number of composition functions which could be applied to the combination of the word vectors in the prior history.

The elements of the language model that have been defined so far (the construction of the history vector and its comparison to upcoming words) are only weakly predictive. The model, as it stands, only gives an indication of which words are coherent with the subject matter so far, and takes no account of the structure or order these words are likely to be found in. Typically, the scheme is extended by integrating the semantic language model with an $n$-gram model. Bellegarda (2000) and Gildea and Hofmann (1999) both use a rescaling approach to combine the probabilities of the two models:

$$
p\left(w \mid h_{1}, h_{2}\right) \propto p\left(w \mid h_{1}\right) \frac{p\left(w \mid h_{2}\right)}{p(w)}
$$

where $h_{1}$ is the $n$-gram representation of history and $h_{2}$ is the semantic representation of history. In other words, the $n$-gram model is rescaled by a factor based on the ratio of the semantic probability to the unigram probability. This can be shown to be equivalent to an assumption that $h_{1}$ and $h_{2}$ are independent. Coccaro and Jurafsky (1998) instead base their integration on the geometric mean of the two models. They also incorporate 
a confidence factor for determining the strength of the semantic models contribution.

Extensions on the basic semantic language models sketched above involve representing the history by multiple LSA models of varying granularity in an attempt to capture topic, subtopic, and local information (Zhang and Rudnicky, 2002); incorporating syntactic information by building the semantic space over words and their syntactic annotations (Kanejiya et al., 2004); and treating the LSA similarity as a feature in a maximum entropy language model (Deng and Khundanpur, 2003).

Instead of constructing separate semantic and $n$-gram models which then need to be combined in some way, Wallach (2006) integrates a topic model with a bigram model directly. Whereas a standard LDA topic model expresses the content of a document as a mixture of topics, with each topic being a unigram distribution over the vocabulary, Wallach (2006) expresses the content of a document as a mixture of bigrams. In this formulation, as in LDA, each document is associated with a distribution over topics, but the topics in this case are bigram distributions, and in this way the model captures both the local bigram structure and the topical semantic structure in one model.

\subsubsection{Connectionist Language Models}

The language models discussed so far in this section have exploited a set of dependencies which were fixed in advance: e.g., to the head of a phrase or to the most recent previous words. Connectionist approaches to modelling the structure of language have taken a different approach by implementing distributed representations that learn which features and dependencies are most useful.

Rather than being concerned with optimising a probabilistic model of word sequences for application to some practical task, early connectionist work on language was often motivated by theoretical criticisms from the symbolic camp (Fodor and Pylyshyn, 1988). This led to a focus on the question of how linguistic structures could be represented in distributed representations and whether these representations could be learned. For example, Elman (1991) applied a simple recurrent network to the 
problem of predicting the next word in sentences generated by a toy grammar. Examination of the network's hidden units showed that the representation learned to encode both lexical and grammatical information.

In contrast, the concern of Bengio et al. (2003) is directed to the practical benefits of connectionist models, rather than their theoretical representational capacities. In this case distributed representations are employed to reduce the free parameters in an $n$ gram model and attain better generalisation. Each word in the vocabulary is associated with a vector, and $n-1$ of these vectors form the input to the network, representing the $n$-gram context. These vectors and the function which predicts the next word from them are learnt during training by stochastic gradient ascent on the log-likelihood of the training data. The network itself has a feed forward architecture, with a single layer of hidden units that combines the input word vectors to form a merged representation which is used to predict probabilities for the next word. This combination of word vectors has something in common with our vector composition models, being a way of integrating word vectors to produce a single representation of a multi-word structure. One major difference, however, is that the word vectors in this case are not designed to represent the semantic properties of the words.

Mnih and Hinton (2007) propose a log-bilinear model in which, again, each word in the vocabulary is associated with a distributed representation. However, in this case the prediction of upcoming words is not simply made in terms of a function computed by a feed forward network. Instead, the probability of the next word is based on its similarity, as measured by the dot product, of its word vector to a history vector derived by combining the $n-1$ prior input vectors. These word vectors and the combination function are learnt by the network by maximising the log-likelihood of a training corpus. Here too, although the representations cannot be considered a purely semantic, the issue of vector combination we are considering is relevant.

Both connectionist models build vectors representing individual words and combine them in some way to represent word sequences. The question of how best to 
implement this, specifically for semantic representations, is the general topic of this thesis. Interestingly, both connectionist models make use of general additive functions (Equation 4.2) in their approach to vector combination, without exploring what multiplicative functions (Equation 4.3) could achieve.

\subsection{Vector Composition}

In this section we will develop a language model based on vector composition. As in previous work, the conditional probability of a word given its history will be based on the semantic similarity of that word to the history. In our case, however, we will investigate the influence of vector composition functions in constructing the representation of the history. Where possible, we will make the implementation of the compositional model as close to that presented in Chapter 5 as possible. So, we will use the same definition of vector components and use the same simple additive and multiplicative composition functions. However, lemmatisation will not be appropriate for the current task. Another difference will be in the manner we measure similarity. While cosine similarity was effective in modelling similarity ratings, we will argue that it has a number of drawbacks for this application. Nonetheless, our derivation of the new measure will be a modification of cosine similarity.

Our focus will be on the simplest composition functions: the simple multiplicative and additive functions. An obvious benefit of these functions is that they can be applied in linear order, because they are insensitive to syntax and word order, making them suitable for forming representations of sentence prefixes in a left to right, word by word manner. Applying the syntactic composition functions would require not only setting the parameters for all the syntactic structures in the grammar, but also figuring out how to build representations for the partial trees of sentence prefixes, as opposed to the complete phrases considered in Chapter 5.

In any case, the simple models proved to give robust results in modelling phrase 
similarity judgements. So far we have considered these functions in terms of their semantic and vectorial properties. From the perspective of a language model, however, it becomes important to understand their probabilistic properties. First we show how these two composition functions can be given a probabilistic interpretation, and following that we derive a language model which compositionally constructs a semantic representation of prior context. Experiment 3 then evaluates these language models in terms of their perplexity on a news corpus.

\subsubsection{Vector Composition from a Probabilistic Perspective}

Experiments 1 and 2 examined the ability of vector composition models to simulate human similarity ratings for phrases. We now want to motivate vector composition as a basis for a probabilistic model by giving the simple additive and multiplicative models a probabilistic interpretation.

The vector components we have been using, in the case of both the simple semantic space and the LDA based representation, have the form of a ratio of probabilities:

$$
v_{i}=\frac{p\left(c_{i} \mid w\right)}{p\left(c_{i}\right)}
$$

where $\mathbf{v}$ is a vector representing the word $w$, and $c_{i}$ represents a context word in the case of the simple semantic space, and in the case of LDA a topic. This component definition was adopted because it performed well in modelling similarity ratings. However, we can also examine the probabilistic properties of these components in relation to the composition functions we are considering.

Let us assume vectors $\mathbf{u}$ and $\mathbf{v}$ represent target words $w_{1}$ and $w_{2}$. Now, when we compose these vectors using the multiplicative model and the components definition in (6.7), we obtain:

$$
h_{i}=v_{i} \cdot u_{i}=\frac{p\left(c_{i} \mid w_{1}\right)}{p\left(c_{i}\right)} \frac{p\left(c_{i} \mid w_{2}\right)}{p\left(c_{i}\right)}
$$

Applying Bayes' theorem:

$$
h_{i}=\frac{p\left(w_{1} \mid c_{i}\right) p\left(w_{2} \mid c_{i}\right)}{p\left(w_{1}\right) p\left(w_{2}\right)}
$$


Assuming $w_{1}$ and $w_{2}$ are independent and applying Bayes' theorem again, $h_{i}$ becomes:

$$
h_{i} \approx \frac{p\left(w_{1} w_{2} \mid c_{i}\right)}{p\left(w_{1} w_{2}\right)}=\frac{p\left(c_{i} \mid w_{1} w_{2}\right)}{p\left(c_{i}\right)}
$$

By comparing to (6.7), we can see that the expression on the right hand side gives us something akin to the vector components we would expect when our target is the co-occurrence of $w_{1}$ and $w_{2}$. Thus, for the multiplicative model, the combined vector $h_{i}$ can be thought of as an approximation to a vector representing the distributional properties of the phrase $w_{1} w_{2}$.

If multiplication results in a vector which is something like the representation of $w_{1}$ and $w_{2}$, then addition produces a vector which is more like the representation of $w_{1}$ or $w_{2}$. Suppose we were unsure whether a word token $x$ was an instance of $w_{1}$ or of $w_{2}$. It would be reasonable to express the probabilities of context words around this token in terms of the probabilities for $w_{1}$ and $w_{2}$, assuming complete uncertainty between them:

$$
p\left(c_{i} \mid x\right)=\frac{1}{2} p\left(c_{i} \mid w_{1}\right)+\frac{1}{2} p\left(c_{i} \mid w_{2}\right)
$$

Therefore, we could represent $x$ with a vector, based on these probabilities, having the components:

$$
x_{i}=\frac{1}{2} \frac{p\left(c_{i} \mid w_{1}\right)}{p\left(c_{i}\right)}+\frac{1}{2} \frac{p\left(c_{i} \mid w_{2}\right)}{p\left(c_{i}\right)}
$$

Which is exactly the vector averaging approach to semantic composition.

Thus, these two composition models have distinct probabilistic interpretations. The multiplicative model is associated with the conjunction of its constituents, that is it treats the pair as a phrase. Whereas, the additive model is based on disjunction, treating the constituents as separate. The success of multiplication or addition as a model of composition is likely then to depend on the appropriateness of conjunction and disjunction in the underlying scheme in which vectors are constructed. 


\subsubsection{Deriving a Language Model}

Our aim now is to derive probabilities, $p(w \mid h)$, given the semantic representations of a word, $w$, and its history, $h$, based on the assumption that probable words should be semantically coherent with the history. Semantic coherence may be measured in terms of similarity, which is commonly calculated via the cosine of the angle between two vectors:

$$
\begin{gathered}
\operatorname{sim}(\mathbf{w}, \mathbf{h})=\frac{\mathbf{w} \cdot \mathbf{h}}{|\mathbf{w}||\mathbf{h}|} \\
\mathbf{w} \cdot \mathbf{h}=\sum_{i} w_{i} h_{i}
\end{gathered}
$$

where $\mathbf{w} \cdot \mathbf{h}$ is the dot product of $\mathbf{w}$ and $\mathbf{h}$. Coccaro and Jurafsky (1998) utilize this measure in their approach to language modeling. Unfortunately, they find it necessary to resort to a number of ad-hoc mechanisms to turn the cosine similarities into useful probabilities. The primary problem with the cosine measure is that its values do not sum to 1 , as probabilities must. Thus, normalization over the entire vocabulary is required. A further problem concerns the fact that such a measure takes no account of the underlying frequency of $w$, which is crucial for a probabilistic model. For example, encephalon and brain are roughly synonymous, and may be equally similar to some context, but brain may nonetheless be much more likely, as it is generally more common.

A better measure would take account of the underlying probabilities of the elements involved and produce values that sum to 1 . Our approach is to modify the dot product (Equation 6.14) on which the cosine measure is based. Assuming that our vector components are given by Equation 6.7, the dot product becomes:

$$
\mathbf{w} \cdot \mathbf{h}=\sum_{i} \frac{p\left(c_{i} \mid w\right)}{p\left(c_{i}\right)} \frac{p\left(c_{i} \mid h\right)}{p\left(c_{i}\right)}
$$

which we modify to derive probabilities as follows:

$$
p(w \mid h)=p(w) \sum_{i} \frac{p\left(c_{i} \mid w\right)}{p\left(c_{i}\right)} \frac{p\left(c_{i} \mid h\right)}{p\left(c_{i}\right)} p\left(c_{i}\right)
$$


This expression now weights the sum with the independent probabilities of the context words and the word to be predicted. That this is indeed a valid probability can be seen by the fact it is equivalent to $\sum_{i} p\left(w \mid c_{i}\right) p\left(c_{i} \mid h\right)$. However, in constructing a representation of the history $h$, it is more convenient to work with Equation 6.16 as it is based on vector components (Equation 6.7) and can be readily used with the composition models we are considering.

Equation 6.16 allows us to derive probabilities from vectors representing a word and its prior history. We must also construct a representation of the history up to the $n$th word of a sentence. To do this, we combine, via some (additive or multiplicative) function $f$, the vector representing that word with the vector representing the history up to $n-1$ words:

$$
\begin{gathered}
\mathbf{h}_{n}=f\left(\mathbf{w}_{n}, \mathbf{h}_{n-1}\right) \\
\mathbf{h}_{1}=\mathbf{w}_{1}
\end{gathered}
$$

One issue that must be resolved in implementing Equation 6.17 is that the history vector should remain correctly normalized. In other words, the products $h_{i} \cdot p\left(c_{i}\right)$ must themselves be a valid distribution over $c_{i}$. So, after each vector composition the history vector is normalized over the vector components as follows:

$$
h_{i}=\frac{\hat{h}_{i}}{\sum_{j} \hat{h}_{j} \cdot p\left(c_{i}\right)}
$$

Equations 6.16- 6.19 define a language model that incorporates vector composition. To generate probability estimates, it requires a set of word vectors whose components are based on the ratio of probabilities described by Equation 6.7.

\subsubsection{Integrating with Other Language Models}

The model defined above is based on little more than semantic coherence. As such it will be only weakly predictive, since word order is largely ignored, which $n$-gram models exploit. The simplest means to integrate semantic information with a standard 
language model involves combining two probability estimates as a weighted sum:

$$
p(w \mid h)=\lambda_{1} p_{1}(w \mid h)+(1-\lambda) p_{2}(w \mid h)
$$

Linear interpolation is guaranteed to produce valid probabilities, and has been used, for example, to integrate syntactic language models with $n$-gram models (Roark, 2001). However, it will work best when the models being combined are comparably predictive and have complementary strengths and weaknesses. If one model is much weaker than the other, linear interpolation will typically produce a model of intermediate strength (i.e., worse than the better model), with the weaker model contributing a form of smoothing at best.

Instead, we use a rescaling approach (Kneser et al., 1997; Gildea and Hofmann, 1999). Based on Equation 6.16, we can express our semantic probabilities as the product of the unigram probability, $p(w)$, and a semantic component, $\Delta$, which determines the factor by which this probability should be scaled up or down given the context in which it occurs.

$$
\begin{gathered}
p(w \mid h)=p(w) \cdot \Delta(w, h) \\
\Delta(w, h)=\sum_{i} \frac{p\left(c_{i} \mid w\right)}{p\left(c_{i}\right)} \frac{p\left(c_{i} \mid h\right)}{p\left(c_{i}\right)} p\left(c_{i}\right)
\end{gathered}
$$

Thus, it seems reasonable to integrate the $n$-gram model by replacing the unigram probabilities with the $n$-gram versions.

$$
\hat{p}\left(w_{n}\right)=p\left(w_{n} \mid w_{n-2}^{n-1}\right) \cdot \Delta\left(w_{n}, h\right)
$$

This is equivalent to assuming that $h$ is conditionally independent of $w_{n-2}^{n-1}$.

This rescaling is only applied to content words, as the semantic representations contain little useful information for function words. To obtain a true probability estimate, $\hat{p}\left(w_{n}\right)$ needs to be normalised, but the rescaling has to be carried out only on content words, with function words retaining the straight $n$-gram prediction:

$$
p\left(w_{n} \mid w_{n-2}^{n-1}, h\right)=\hat{p}\left(w_{n}\right) \frac{\sum_{w_{c}} p\left(w_{c} \mid w_{n-2}^{n-1}\right)}{\sum_{w_{c}} \hat{p}\left(w_{c}\right)}
$$


where the sum over $w_{c}$ is a sum over content words only. In other words, $\hat{p}\left(w_{n}\right)$ is normalised so that the total probability mass for content words is equal to the probability mass assigned to content words by the $n$-gram model. This leaves the remaining probability mass assigned to function words unchanged.

In integrating our semantic model with an $n$-gram model, we allow the latter to handle short range dependencies and have the former handle the longer dependencies outside the $n$-gram window. For this reason, the history $h$ used by the semantic model in the prediction of $w_{n}$ only includes words up to $w_{n-3}$ (i.e., only words outside the $n$-gram).

We also integrate our models with a syntactic language model (Roark, 2001), as this allows us to compare the benefits derived from incorporating long-range syntactic dependencies to those derived from our semantic model. However, in this case we use linear interpolation (Equation 6.20) because the models are roughly equally predictive and also because linear interpolation is widely used when probabilistic parsers are combined with $n$-grams and other information sources. This approach also has the benefit of allowing the models to be combined without out the need to renormalize the probabilities. In the case of the parser, normalizing across the whole vocabulary would be prohibitive.

\subsection{Experiment 3}

\subsubsection{Method}

We experimented with additive and multiplicative composition functions, and two semantic representations (LDA and the simpler semantic space model), resulting in four compositional models. In addition to implementing the stand alone semantic compositional model, we integrated this model with a standard $n$-gram model (see Equation 6.23). We also compared our models against a state of the art syntactic language model in order to assess the extent to which the information provided by the seman- 
tic representation is complementary to syntactic structure. Our experiments used the grammar-based language model of Roark (2001), which is suitable for integration with the semantic and $n$-gram components because of its incrementality.

All our models were evaluated by computing perplexity ${ }^{1}$ on a test set. Roughly, this quantifies the degree of unpredictability in a probability distribution, such that a fair $k$-sided dice would have a perplexity of $k$. More precisely, perplexity is the reciprocal of the geometric average of the word probabilities and a lower score indicates better predictions. We calculated this measure by taking negative logs of the probabilities, averaging them, and then exponentiating that value:

$$
\text { perplexity }=\prod p^{-1 / N}=x^{\Sigma-\log _{x}(p) / N}
$$

Although this is a commonly used metric for evaluating language models there is no established method of testing the significance of differences in this measure. Here, we chose to not only report a single overall perplexity for the whole test set but to also perform significance tests at the level of individual words, where we could identify a range of variation within the results for each model and compare that to the variation between models. It would be possible to calculate per-word perplexities but these values have an extremely skew distribution, and it is difficult to decide what should be tested and how that test relates to the overall perplexity values. Instead, we performed a paired $t$-test on the value $-\log (p)$, which is the $\log$ of the per-word perplexity, and these values have a distribution much closer to normality. The $t$-test tests for a difference in means and the mean of $-\log (p)$ is just the $\log$ of the overall perplexity, giving a fairly direct relationship between the hypothesis test and the perplexity values.

\footnotetext{
${ }^{1}$ Word error rate is an alternative measure for evaluating language models. Essentially this quantifies the benefit the model brings to a speech recognition system. Since this requires integration with an acoustic model, and we are not directly interested in speech recognition anyway, we choose to use perplexity as our evaluation metric.
} 


\subsubsection{Data}

The experiments were based on data from the Wall Street Journal in the BLLIP corpus (years 1987-89) and Penn Treebank (1989). We pre-processed and partitioned the BLLIP corpus into training, test and development sets as described in Chapter 3. Specifically, numbers were replaced with the symbol $\langle$ num $\rangle$, and a vocabulary of 20,000 words was chosen and the remaining tokens were replaced with $\langle$ unk $\rangle$. From this data, a 38,521,346 word training set was used to construct the trigram language model and the semantic representations, while perplexity results were calculated on a 50,006 word test set ${ }^{2}$. The Penn Treebank was pre-processed in the same way, with the whole treebank being used as a training set for the syntactic language model.

\subsubsection{Model Parameters}

The semantic components of the language models were based on the simple semantic space and LDA model constructed on the BLLIP corpus described in Chapter 3. Our composition models were the unparametrised simple additive and multiplicative functions.

We integrated our compositional models with a trigram model which we also trained on BLLIP. The model was built using the SRILM toolkit (Stolcke, 2002) with backoff and modified Kneser-Ney smoothing (Chen and Goodman, 1999). Following previous work on syntactic language modeling (Roark, 2001; Charniak, 2001; Chelba and Jelinek, 1998), we trained the parser on sections 2-21 of the Penn Treebank containing 936,017 words. Note that Roark's (2001) parser produces prefix probabilities for each word of a sentence which we converted to conditional probabilities by dividing each current probability by the previous one. 


\begin{tabular}{|l|c|}
\hline Model & Perplexity \\
\hline Unigram & 2604 \\
Add $_{\text {SSM }}$ & 2099 \\
Multiply $_{\text {SSM }}$ & 1734 \\
Add $_{\text {LDA }}$ & 1593 \\
Multiply $_{\text {LDA }}$ & 6659 \\
\hline
\end{tabular}

Table 6.1: Perplexities for unigram, and compositional language models on content words in the test set; subscripts SSM and LSA refer to the semantic space and LDA models, respectively.

\subsubsection{Results}

Table 6.1 shows perplexity results for the stand alone semantic models. Because these models are based purely on semantic similarity they are applied only to the 26,583 content words in the test set, and not function words such as the and with. With regard to the simple semantic space model (SSM), we observe that both additive and multiplicative approaches to constructing history are successful in reducing perplexity over the unigram baseline, with the multiplicative model outperforming the additive one. A $t$-test confirms that these differences are significant $(p<0.01)$. Since the unigram model takes no history into account, this demonstrates that these models successfully capture at least some of the semantic dependencies within the test sentences.

The results for the LDA model are also reported in the table. This model reduces perplexity with an additive composition function, but performs worse than the unigram with a multiplicative function. Again, these differences are significant $(p<0.01)$. The additive LDA model also significantly $(p<0.01)$ outperforms the simple semantic space models and is the best performing model overall.

Table 6.2 reports the perplexities for these semantic models when they are inte-

\footnotetext{
${ }^{2}$ The test set was drawn from the year 1987 to avoid overlap with the Penn Treebank.
} 


\begin{tabular}{|c|c|}
\hline Model & Perplexity \\
\hline$n$-gram & 77.42 \\
\hline$n$-gram $+\operatorname{Add}_{\mathrm{SSM}}$ & 75.24 \\
\hline$n$-gram + MultiplyssM & 73.77 \\
\hline$n$-gram $+\operatorname{Add}_{\mathrm{LDA}}$ & 73.06 \\
\hline$n$-gram+MultiplyLDA & 143.66 \\
\hline parser & 173.35 \\
\hline$n$-gram + parser & 75.23 \\
\hline$n$-gram + parser + Add $_{S S M}$ & 73.27 \\
\hline$n$-gram + parser + MultiplysSM $_{\text {SSM }}$ & 71.27 \\
\hline$n$-gram + parser $+\operatorname{Add}_{L D A}$ & 70.02 \\
\hline$n$-gram + parser + Multiply ${ }_{\text {LDA }}$ & 91.08 \\
\hline
\end{tabular}

Table 6.2: Perplexities for $n$-gram, composition and syntactic language models, and their combinations on the full test set; subscripts SSM and LSA refer to the semantic space and LDA models, respectively.

grated with an $n$-gram model. In this case, the perplexities are for the whole vocabulary, with the $n$-gram probabilities for function words left unaltered but content words rescaled by the semantic model. Here, the results follow much the same pattern as before with the multiplicative model producing significantly $(p<0.01)$ better results for the simple semantic space and the additive LDA model being significantly $(p<0.01)$ better than all the other models including the simple semantic space models. Comparison to the plain $n$-gram baseline shows that the semantic models are successful in exploiting semantic dependencies to words outside the $n$-gram window, except notably for the multiplicative LDA model.

For comparison, Figure 6.1 plots the perplexity of the combined LDA and $n$-gram models against the number of topics. Increasing the number of topics produces higher 


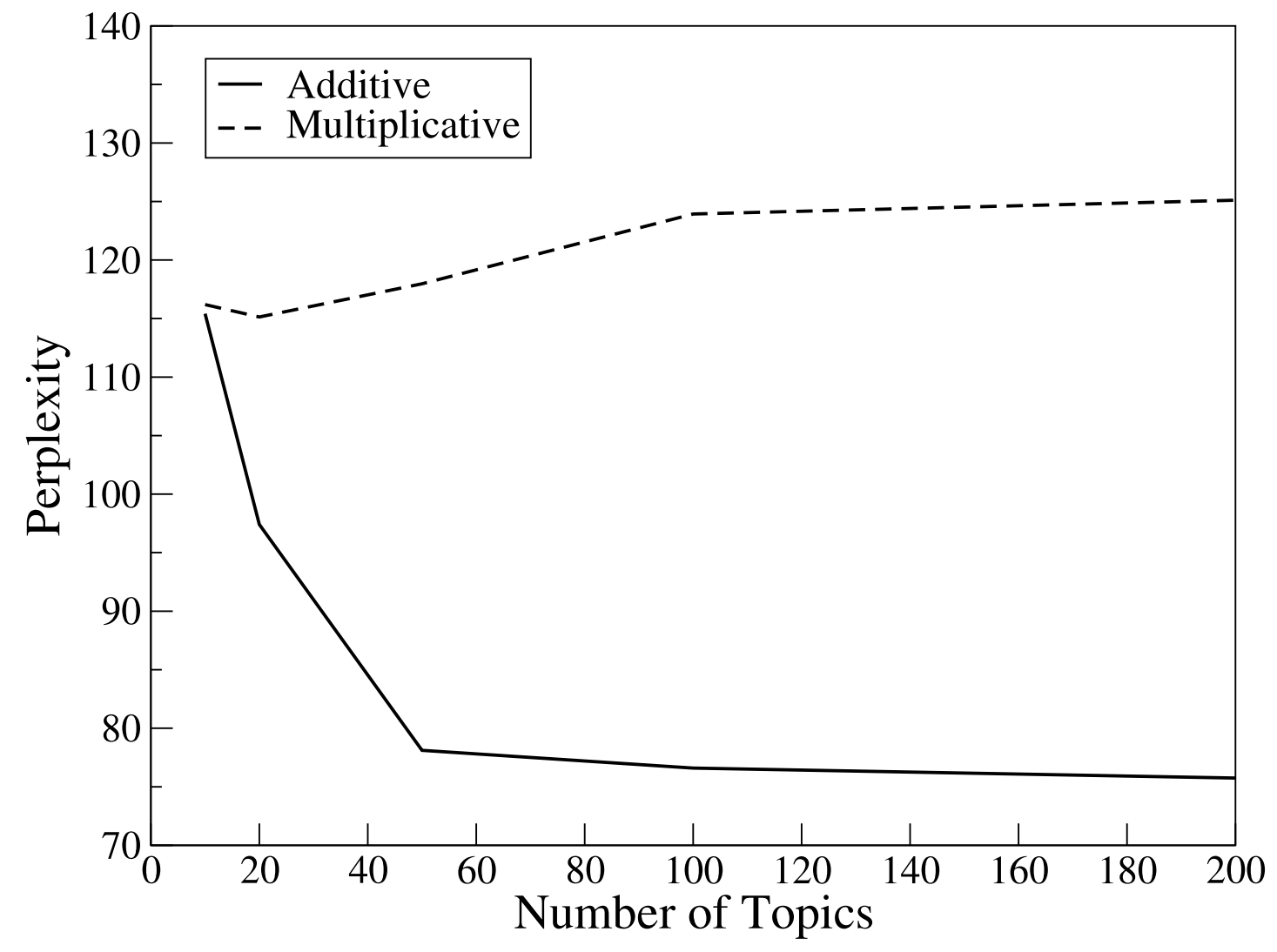

Figure 6.1: Perplexity versus Number of Topics for the LDA models using additive and multiplicative composition functions.

dimensional representations which ought to be richer, more detailed and therefore more predictive. While this is true for the additive model, a greater number of topics actually increases the perplexity of the multiplicative model, indicating it has become less predictive.

We compared these perplexity reductions against those obtained with a syntactic language model. Following Roark (2001), we combined the probabilistic parser with a trigram model using linear interpolation (the weights were optimized on the development set). This model ( $n$-gram + parser $)$ is significantly $(p<0.01)$ outperformed by our best compositional models ( $n$-gram + MultiplysSM, $n$-gram $\left.+\operatorname{Add}_{\text {LDA }}\right)$. While both models incorporate long range dependencies, the parser is trained on a hand annotated treebank, whereas the compositional model uses raw text, albeit from a larger cor- 
pus. Furthermore, three interpolated compositional models $\left(n\right.$-gram + parser $+\operatorname{Add}_{\mathrm{SSM}}$, $n$-gram + parser + Multiply $_{S S M}$ and $n$-gram + parser $\left.+\operatorname{Add}_{\text {LDA }}\right)$ are all also significantly better then the interpolated model without composition $(n$-gram + parser $)$ This suggests that these semantic models are encoding useful predictive information about long range dependencies, which is distinct from and potentially complementary to the parser's syntactic information about such dependencies. The interpolated additive LDA model $\left(n\right.$-gram + parser $\left.+\operatorname{Add}_{\text {LDA }}\right)$ significantly $(p<0.01)$ outperforms all the other models.

\subsubsection{Discussion}

Our results indicate that the compositional models are successful in representing semantic dependencies both on their own and in combination with an $n$-gram model. Moreover, by integrating with a parser we achieved further perplexity reductions, demonstrating that these syntactic and semantic components exploit distinct long range dependencies.

For the simple semantic space, both the additive and multiplicative models produced predictive representations. Interestingly, in the LDA setting only the additive model produced improvements over the baseline with the multiplicative model proving detrimental. Increasing the representational power of the LDA model, by using a greater number of topics, rendered the multiplicative model less predictive.

Given this lack of performance, we would like to know why the multiplicative model is not effective when applied to the LDA representation. In particular, Equations 6.8 to 6.10 of Section 6.2.1 appear to show that the multiplicative model can be derived from some simple assumptions. However, our experimental results demonstrate that this derivation is not valid for LDA.

The problem arises because in the LDA model each word of a phrase may be generated by a distinct topic. The representation of the whole phrase, therefore, should contain all these topics. However, the derivation of the multiplicative model assumes 
that the topics which are relevant to the whole phrase are those that could generate all its constituents. Addition by contrast was related in Section 6.2.1 to a disjunction of the words in a phrase, and in this way is more effective at gathering the disjoint topics associated with the words in a phrase into a single representation.

The multiplicative model is much more successful on the simple semantic space. We can explain this in terms of the fact that if a context word is associated with a whole phrase, it is likely to be associated with each of the constituents. In other words if $c$ never appears in the context around $w$, it is unlikely to appear in the context surrounding any phrase containing $w$.

More pragmatically, the effectiveness of these compositional models is related to the sparsity of the underlying vector representations. LDA representations tend to be fairly sparse, and multiplication increases this sparsity resulting in useful information being discarded. Whereas, the simple semantic space representations are much more distributed and multiplication in this case is effective in whittling down these vectors to the most relevant components.

\subsection{Conclusions}

In this chapter we demonstrated that a language modelling task could be used to evaluate the ability of compositional representations to capture the semantic dependencies in natural text. We constructed representations of prior history compositionally and derived probabilities for upcoming words based on the assumption that they should be similar to that history. We evaluated this as a stand alone model and also in combination with an $n$-gram model and a syntactic model. Our results are broadly in agreement with those of Chapter 5, with the multiplicative model being most successful for the simple semantic space and the additive model being better for the LDA model.

Neither of these forms of semantic representation is in fact designed for the language modelling task. It would be interesting therefore to develop representations 
which are derived from the language model outlined in Section 6.2.2. That is, we could treat the components of word vectors as parameters in the probabilistic model defined by Equations 6.16 to 6.19. Optimising these parameters on the likelihood of this model would allow us to find word representations which are adapted to the language modelling task. This would also have the benefit that the representations would be constructed in a manner that takes into account the composition function that combines them.

Another extension of the work presented in this chapter would be to integrate vector composition within a syntactic language model. The current model, for the purposes of incrementality, is based on word order, as opposed to syntactic structure. However, as our ultimate goal is to develop a syntax aware approach to vector composition, embedding these models in a probabilistic grammar would be desirable.

Having shown that vector composition has practical applications in language modelling, we would now like to show that these models are cognitively relevant; that they are related to the cognitive processes of real language users. One way to gain insight into these cognitive processes is to exploit the information about processing load that is revealed in reading times. In particular, greater cognitive loads, and therefore longer reading times, are associated with anomalous or unexpected inputs. The language models of this chapter permit us to derive a precise measure of how semantically and syntactically expected an input word is given its history based on its probability of occurence. In Chapter 7 we will examine the use of these models in analysing reading times as revealed in an eye movement study, and the extent to which the distributional models of composition contribute to the prediction of processing load. 


\section{Chapter 7}

\section{Predicting Eye-movements in Reading}

This chapter describes our experiments on the Dundee corpus of eye-movement data, assessing the utility of our approach to semantic composition in modelling the influence of semantic constraint on language comprehension processes. Chapter 6 showed that compositional representations of prior semantic content could be used to predict upcoming words within a language model. Here we relate these semantic predictions to measures of processing difficulty associated with reading text. Reading times, as revealed by eye-tracking studies, provide an insight into the cognitive load generated in processing words in context, with longer reading times indicating greater processing effort. One determinant of this processing load is the degree of semantic constraint; the extent to which upcoming words are predictable from their context. Modelling this effect requires some way of constructing a representation of the prior context and quantifying the relation between this context and upcoming words. Our distributional representations and composition functions are therefore ideal candidates for use in modelling semantic constraint. Experiment 4 integrates semantic and other effects into a single surprisal based measure of processing cost derived from the language models of the previous chapter, and investigates different approaches to composing vectors for constructing the representation of semantic context. 


\subsection{Cognitive Processes and Eye-movements in Read- ing}

A wealth of evidence demonstrates the strong relation between eye-movements and cognitive activity during reading (Rayner, 1998). In particular, the amount of time spent reading a particular piece of text gives some insight into the overall cognitive load associated with processing it. Here, we wish to investigate the cognitive plausibility of our compositional representations by analysing their effectiveness as predictors of the processing load associated with long range semantic dependencies. It is well known that input which is semantically coherent with the previous context is read more quickly than anomalous input (Stanovich and West, 1981). In Chapter 6, we derived language models that exploited our compositional representations to incorporate the effects of semantic coherence, and evaluated them in terms of their perplexity on a test set. In this chapter, we will investigate the effects on reading of these coherence effects, deriving a measure of processing cost from our integrated language model and evaluating it as a predictor of reading times.

To do this, we will use the eye-movement data contained in the Dundee corpus (Kennedy and Pynte, 2005). However, because this data comes from an eye-tracking study of self-paced reading of natural text, rather than a controlled experiment that isolates a single effect of interest, the reading times are subject to a number of confounding factors which need to be accounted for. In the following discussion we will outline the general characteristics of eye-movements and discuss some of the factors which affect them.

\subsubsection{Eye-movements}

The activity of the eye during reading can be broken down, at a gross level, into two main components: fixations (on a timescale of hundreds of milliseconds) and rapid movements between fixations known as saccades (of the order of tens of millisec- 
onds). Most of these movements follow the direction of text in a left to right manner jumping around 7 to 9 letters. However, $10 \%$ to $15 \%$ of saccades are regressions in the right to left direction, returning to prior input. During all saccades, input from the eyes is suppressed (Campbell and Wurtz, 1978; Riggs et al., 1974), and so visual information is gathered by readers entirely during fixations. It has been shown that the required information for normal reading can be acquired within around 50ms (Rayner et al., 1981), so the remainder of fixation time is available for further processes: e.g., lexical access, semantic integration and planning subsequent saccades. Although all the required visual information is obtained during fixations, many words are skipped entirely, with only $35 \%$ of function words being fixated, compared to $85 \%$ for content words (Carpenter and Just, 1983). At least some of the information about unfixated words is acquired from parafoveal input. During fixations, information is taken in from an asymmetric visual field of limited extent. This perceptual span covers at most 3 or 4 letters to the left, while to the right it extends to 14 or 15 characters (McConkie and Rayner, 1976, 1975). However, information gained about words to the right of that being fixated consists mainly of its first 3 letters and total word length (Rayner et al., 1982; Lima and Inhoff, 1985).

Eye-tracking studies can thus provide a rich source of information about the timecourse of processing textual information. Comprehension processes in reading can be studied in terms of whether words are skipped or fixated (Brysbaert and Vitu, 1998), when regressions occur (Vitu et al., 1998) and how long fixations last (Liversedge et al., 1998). This latter aspect will be the focus of the work presented here. However, multiple measures of fixation time have been proposed and analysed in previous research: first fixation duration (the duration of the first fixation on a word regardless of whether it is the first fixation on a word or the first of multiple fixations on the same word), first pass duration, also known as gaze duration, (the sum of all fixations made on a word prior to looking at another word), and total reading time (the sum of all fixations on a word including refixations after moving on to other words). One hypothesised dif- 
ference between these times is the distinction between early and late measures. Early measures, such as first fixation duration, are more strongly driven by the initial analysis of a word, reflecting factors such as visual recognition and lexical access, whereas late measures, such as total reading time, reflect high level processes of integration and comprehension. Given that we are interested here in the influence of semantic dependencies, we will focus on total reading time in our analyses. It is also worth noting that although the total time spent reading an entire text is indicative of the total processing cost for that text, the breakdown of this relationship to a word-by-word basis is less reliable, due to spillover effects (Rayner and Duffy, 1986). It is entirely conceivable that processing of prior input continues after a saccade has been made onto a new fixation. Nonetheless, fixation times have been an extremely useful tool in probing the processing difficulty associated with specific regions within a text.

\subsubsection{Cognitive Load}

A number of factors have been investigated as sources of cognitive load during reading. Infrequent or unfamiliar words incur greater processing costs, in terms of processing them both as isolated words (Cattell, 1886) and also in context (Inhoff and Rayner, 1986; Rayner and Raney, 1996). In context, the first occurrence of a word incurs the greatest processing cost, with subsequent reading times decreasing (Rayner et al., 1995). Similarly, the introduction of new discourse referents incurs a processing cost (Haviland and Clark, 1974; Garrod and Sanford, 1994), and the resolution of pronouns to these referents also incurs a cost (Ehrlich and Rayner, 1983). It is also hypothesised that an integration cost is associated with incorporating each word into the structure built so far (Gibson, 2000), for example integrating a verb with its arguments. Evidence also indicates that the extent to which this partial structure constrains the subsequent input affects the processing load (Ehrlich and Rayner, 1981; Rayner and Well, 1996), with more constrained words having shorter fixations and being skipped more. Two competing explanations can be used to explain these constraint effects (Kamide, 2008). 
The first is that highly constraining contexts allow the prediction of their completions and processing of these predicted items is then facilitated. The second is that the integration cost for integrating such input into a highly constraining context is reduced. Unfortunately, reading times by themselves cannot distinguish between these prediction and integration hypotheses. To investigate this issue more deeply, in particular to identify whether the language processor is capable of making the necessary predictions required for the former explanation, a number of reasearchers have turned to the visual world paradigm (Cooper, 1974; Tanenhaus et al., 1995; Altmann and Kamide, 1999). In this approach, linguistic input is paired with a visual context in which potential referents are located, with an eye-tracking setup allowing the identification of the subjects attention during the experiment. For example, Altmann and Kamide (1999) presented participants with the image in Figure 7.1, which contains a boy, a cake, a toy car, a toy train and a ball, while they heard sentences such as:
(1) a. The boy will eat the cake.
b. The boy will move the cake.

They found that, on hearing the verb, a significantly greater number of subjects made anticipatory eye-movements towards an object (i.e. the cake) when this target could be predicted from the verb (i.e. eat) in comparison to when it wasn't (i.e. move). This shows that subjects are indeed capable of predicting the arguments of verbs before they hear them. In further experiments, Kamide et al. (2003) showed that such argument prediction could occur even before the relevant head had been encountered, in Japanese, a head-final language. Nonetheless, although these experiments show that subjects can and do make highly specific predictions about upcoming input, they do not tell us what effect these predictions have on reading times. The question remains of how prediction and integration effects contribute to cognitive load. 


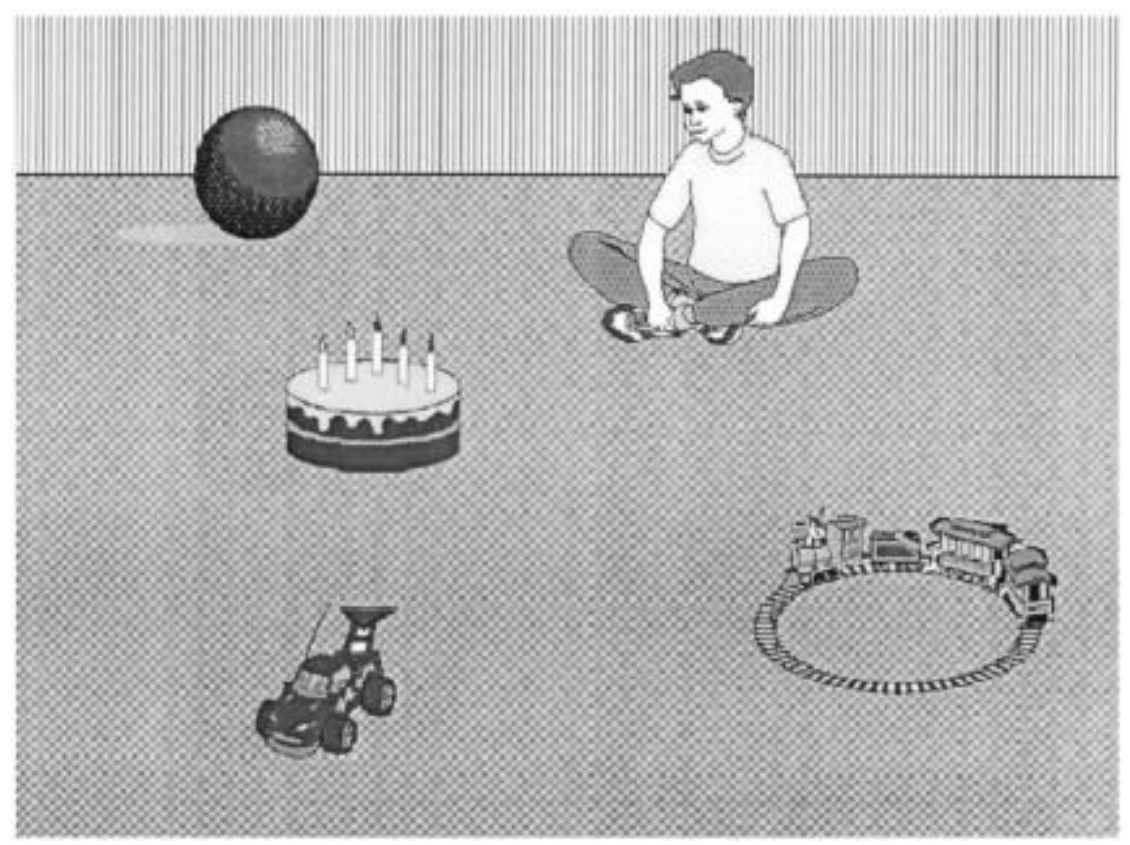

Figure 7.1: Visual display from Altmann and Kamide (1999).

\subsubsection{Integration and Prediction Costs}

Discourse Locality Theory (DLT, Gibson 2000) attempts to quantify the processing costs associated with the integration of heads and dependents in terms of the distance between them. The main assumption of this proposal is that integration costs are a function of the complexity of the intervening material. In particular, processing cost increases as the number of new discourse referents between the head and dependent increases. This proposal can be used to explain why object extracted relative clauses are more difficult to process than subject extracted relative clauses, and why object extracted relative clauses with pronoun subjects are easier to process than those with full noun phrases.

To quantify the processing costs associated with the predictability of the input, Hale (2001) has proposed surprisal, a measure derived from a probability distribution over word strings. Hale (2001) suggests that the language processor maintains expectations over possible continuations of the current input. These expectations are essentially probability distributions over word strings consistent with what has been seen so far. 
As new input is processed, these probability distributions change, and the magnitude of this change corresponds to the processing difficulty associated with that input. Hale (2001) proposes, specifically, that the level of cognitive load is related to the proportion of probability mass which is disconfirmed by the new input. This is quantified in terms of the log of the ratio of the previous probability mass to the new mass. So, the surprisal for $w_{n+1}$ is given by:

$$
\text { Surprisal }=\log \frac{p\left(w_{0} w_{1} w_{2} \ldots w_{n}\right)}{p\left(w_{0} w_{1} w_{2} \ldots w_{n} w_{n+1}\right)}
$$

And this can be expressed in terms of the conditional probability of $w_{n+1}$ :

$$
\text { Surprisal }=-\log p\left(w_{n+1} \mid w_{0} w_{1} w_{2} \ldots w_{n}\right)
$$

Thus, surprisal measures fairly directly the predictability of a word in context, with entirely predictable words $(p=1)$ associated with zero surprisal. In principle, its calculation could be based on any method that assigns probabilities to strings of words, e.g., an $n$-gram language model. Commonly, however, probabilistic parsers have been used in this regard, because the phenomena of interest are frequently the differential loads associated with various syntactic structures.

An alternative derivation of the same measure is suggested by Levy (2008), who argues that resources for alternative interpretations of the current input are allocated in parallel according to the probability of those interpretations. As new words are encountered, resources are re-allocated and this is associated with a processing cost. The size of this cost is determined by the difference between the old expectations and new expectations, which can be measured in terms of the Kullback-Leibler divergence. Levy (2008) shows that this reduces to the same surprisal measure proposed by Hale (2001).

Hale (2001) illustrates the surprisal measure with a small PCFG, calculating the prefix probabilities used in Equation 7.1 using an Earley parser (Stolcke, 1995). He demonstrates that it can be used to explain the processing difficulty encountered at gar- 
den path sentences ${ }^{1}$ and also the increased difficulty associated with object extracted relative clauses in comparison to subject extracted relative clauses. Interestingly, this explanation is made in entirely different terms to DLT's explanation of the same phenomenon. Whereas DLT ascribes the increased difficulty of object extracted relative clauses to an integration cost associated with the complexity of intervening material, the surprisal based explanation appeals simply to the relative frequency of the two constructions; i.e. the subject extracted relative clause is more expected.

Demberg and Keller (2008) consider the contributions of the two formalisms to predictions of eye-movement data. They analysed reading times in the Dundee corpus (Kennedy and Pynte, 2005) in terms of both surprisal and DLT costs, finding that only surprisal was an effective predictor across the whole corpus. While DLT was a significant predictor for subsets of words, such as verbs or nouns, its overall utility was undermined by the fact it assigned zero cost to a large proportion of the corpus.

Roark et al. (2009) also model reading times in terms of surprisal derived from an incremental parser. They partition this overall surprisal measure into syntactic and lexical components and find that both are significant predictors of processing difficulty. Frank (2009) uses both an unlexicalised incremental parser and a simple recurrent neural network to construct language models and derive surprisal measures. He finds that for models of equivalent perplexity, the recurrent network surprisal is a more effective predictor of reading times then the parser, and interprets this result as indicating that the network provides a better model of human language processes.

Surprisal is therefore a robust model of processing costs during reading. However, a number of other measures based on the predictability of the input have been proposed. As discussed, reading times are influenced by unigram word frequencies (Inhoff and Rayner, 1986; Rayner and Raney, 1996), with less frequent words taking longer to process. In addition, McDonald and Shillcock (2003) showed that bigram statistics

\footnotetext{
${ }^{1}$ While hearing a sentence like The horse raced past the barn fell (Bever, 1970), English speakers are inclined to interpret horse as the subject of raced expecting the sentence to end at the word barn. So, upon hearing the word fell they are forced to revise their analysis of the sentence thus far and adopt a reduced relative reading.
} 
also have an effect, for both forward and backward transition probabilities ${ }^{2}$.

Although the measures of processing difficulty associated with the language processor's expectations about the input discussed so far have been based on probabilities, semantic expectations are commonly treated in a different manner. In this case, semantic similarity of new input to the history is often used as a measure of expectedness, with highly similar input assumed to be coherent and expected, while dissimilar input is surprising and semantically anomalous. For example, Pynte et al. (2008) measure semantic coherence in terms of LSA similarities and find this to be a significant predictor of reading times on the French part of the Dundee corpus (Kennedy and Pynte, 2005).

\subsubsection{Low Level Costs}

In addition to factors relating to the high level processing of semantic and syntactic structure, a number of low level costs also contribute to the overall reading time. Word length carries a processing cost, with longer words taking longer to read (Just and Carpenter, 1980). The length of a saccade also affects reading time, as does where the saccade lands in a word (Vitu et al., 2001). Vitu et al. (2001) found that landing positions near the center of a word result in greater reading times than those near the edge, and dubbed this the inverted optimal viewing position effect. As already discussed, spillover effects may also contribute to the reading time for a single word, and so reading time for the previous fixation can also be predictive of reading times for the current fixation.

\subsection{Surprisal for Compositional Language Models}

The compositional language models of Chapter 6 integrate semantic, syntactic and trigram factors to derive the probability of a word given its history. These models

\footnotetext{
${ }^{2}$ Forward transition probability is just the bigram conditional probability of a word given the previous word, whereas the backward probability is the probability of the same word given the next word.
} 
therefore provide an ideal basis for the calculation of a single surprisal measure which takes into account the semantic, syntactic and lexical dependencies which might be used to anticipate upcoming input. Moreover, the effectiveness of this measure as a predictor of eye-movements ought to be informative about the cognitive plausibility of the factors it incorporates. In particular, we will use the evaluation of these factors as predictors of reading times to draw inferences about the validity of our compositional semantic representations as models of comprehension processes during reading. Our assumption is that the more closely our composition processes correspond to the cognitive processes of combining and integrating meanings, the more effective our surprisal measures will be as predictors of reading times. In addition, we would like to confirm that these surprisal measures balance the contribution of the semantic, lexical and syntactic components appropriately. This balance of components for the integrated language model was set largely with the intention of maximising perplexity. In contrast, an integrated measure of processing cost should scale the contributions of its various component factors to match their effect on cognitive load.

We now examine this integrated surprisal measure in more detail, showing how it can be broken down into its component factors to allow the evaluation of their individual contributions. Recall that the integrated language model, $p(w \mid h)$, of Chapter 6 is an interpolation of a compositional-trigram model, $p_{1}$, and a syntactic model, $p_{2}$.

$$
p(w \mid h)=\lambda p_{1}+(1-\lambda) p_{2}
$$

Furthermore, $p_{1}$ is itself the product of a semantic factor, $\Delta$, and a trigram probability, $p_{t}$ :

$$
p_{1}=p_{t} \Delta
$$

Now, the probabilities produced by this language model can be converted to surprisal values:

$$
\text { Surprisal }=-\log p(w \mid h)
$$


Which, given Equation 7.3 can be expressed as:

$$
\text { Surprisal }=-\log \left(\lambda p_{1}+(1-\lambda) p_{2}\right)
$$

We can break this expression down into the sum of two parts:

$$
\text { Surprisal }=-\log \left(p_{1}\right)-\log \left(\lambda+(1-\lambda) \frac{p_{2}}{p_{1}}\right)
$$

Using Equation 7.4, the first term, $-\log \left(p_{1}\right)$, can also be broken down further:

$$
\text { Surprisal }=-\log \left(p_{t}\right)-\log (\Delta)-\log \left(\lambda+(1-\lambda) \frac{p_{2}}{p_{1}}\right)
$$

This breakdown then gives us three components which combine additively to produce the total surprisal. We can then enter these terms into a linear model for reading time, alongside other predictors of processing cost.

$$
\text { Time }=-\beta_{1} \log \left(p_{t}\right)-\beta_{2} \log (\Delta)-\beta_{3} \log \left(\lambda+(1-\lambda) \frac{p_{2}}{p_{1}}\right)+\ldots
$$

If $\beta_{1}, \beta_{2}$ and $\beta_{3}$ are optimised to predict reading times then the relative sizes of these coefficients can be taken as indicating the relative contributions of the trigram, semantic and syntactic factors to the overall cognitive load. While this breakdown allows the weightings of these factors to be set freely, they are constrained to be equal in the case of the integrated surprisal measure. Thus, in the case that $\beta_{1}, \beta_{2}$ and $\beta_{3}$ are all equal to the same value, $\beta_{0}$, Equation 7.9 reduces to a simple relationship between time and surprisal:

$$
\text { Time }=-\beta_{0} \text { Surprisal }+\ldots
$$

In fact, this is equivalent to entering the total integrated surprisal measure as a term itself in the linear model instead of its three separated components. Thus, if the integrated measure balances the contributions of the trigram, semantic and syntactic components appropriately, we should expect that the coefficients, $\beta_{1}, \beta_{2}$ and $\beta_{3}$, of these terms should all be approximately equal to the coefficient, $\beta_{0}$, of the surprisal term on its own. This approximate equality would then indicate that the contributions of the component factors in the integrated measure, chosen for the purposes of language modelling, was also valid in the context of measuring processing cost. 


\subsection{Experiment 4}

\subsubsection{Analysis Methodology}

We evaluated our integrated surprisal measures as predictors of reading times using linear mixed effects models (LME, Pinheiro and Bates 2000). The benefit of these models is that they allow us to handle the systematic differences between subjects in a statistically rigorous manner. The assumption, made by ordinary least squares regression, that samples are independently identically drawn is broken for the eye-tracking data, because we have repeated measures for each subject. That is, the reading times for a single subject are not independent of each other and are likely to be correlated. Thus, rather than applying ordinary regression methods inappropriately to this data, LME methods are recommended for the analysis of reading times (Richter, 2006). This technique allows us to treat subjects as if they were a random factor, sampled from a larger population, and assign an appropriate portion of the variance in the dependent variable to them. Essentially, this means each participant is assigned their own intercept term to account for individual differences in the rates at which they read. LME models also typically contain fixed effects, which are comparable to the independent variables in a standard regression analysis, with each factor being assigned a coefficient which quantifies its effect on the dependent variable. We evaluate the effect of adding a factor to an LME model by comparing the likelihoods of the models with and without that factor. If a $\chi^{2}$ test on the likelihood ratio is significant, then this indicates that the new factor significantly improves model fit. The parameters of the model are set by an iterative procedure which optimises the likelihood of the model and this process is most effective when all the variables are centred and decorrelated from each other. The overall approach we took was to construct a baseline model of reading times using a set of predictors based on low level factors and on short range dependencies, and then evaluate our surprisal based predictors in terms of their ability to predict the remaining variance in reading times. 


\subsubsection{Data}

Two sources of data were used in the analysis presented here. The semantic representations and language models were constructed on the BLIIP corpus, as described in the previous chapter and we also calculated word frequency and bigram statistics from this data. We then evaluated these as predictors of reading times, which were derived from the English portion of the Dundee Corpus (Kennedy and Pynte, 2005), containing eye-tracking data for 10 English native speakers on 20 texts. These texts are drawn from from The Independent newspaper, consisting of 51,502 tokens and 9,776 types in total. Following the methodology of Demberg and Keller (2008), this eye-movement data was preprocessed by first removing the first and last words on each line of visual input as well as words immediately followed by punctuation, to avoid edge and wrapup effects. Regions where four or more consecutive words had been skipped indicate possible tracking errors or disruption of the subjects attention, and these were excluded too. From this data, we computed total reading time for each word in the corpus. Our statistical analyses were based on actual reading times, and so we only included words that were not skipped. We also excluded words for which the previous word had been skipped, and words on which the normal left-to-right movement of gaze had been interrupted, i.e., by blinks, regressions, etc. Lastly, because our focus is the influence of semantic context, we selected only content words whose prior sentential context contained at least two further content words, resulting in the final set of 53,704 data points.

We performed various transformations on our data to achieve three aims. First, we wanted to avoid highly skew distributions. Second, we attempted to decorrelate, as much as possible, our independent variables. Third, we centred our variables, for preparation for the LME analysis. Skew distributions are associated with a number of problems which are best avoided. Variation contained in the sparse tail of the distribution often dominates the variation in the main body of the distribution, producing outliers which have disproportionate influence. In addition, this sort of structure can 
indicate that the variation in the variable would be better represented on the multiplicative scale. Ideally, we would like the factors we analyse to be measured on a scale in which one unit of variation has the same meaning across the full range of the distribution. Taking the example of pay rises, a change of $\$ 1$ in a total of $\$ 10$ is not really the same as a change of $\$ 1$ in a total of $\$ 1,000,000$. In this case, it would probably make more sense to represent these changes as multiplicative factors, for example percentages. A highly skewed distribution, particularly when it is bounded below by zero, often suggests that the underlying processes are multiplicative. Commonly such distributions are transformed by taking logs. This tends to stretch out the body of the distribution and squeeze its tail, resulting in a more even distribution. This also puts the variable onto a multiplicative scale, in which multiplying the original variable by a given factor results in a fixed additive change in the log. We applied this transformation to reading times, word frequencies, forward and backward bigram probabilities, launch distance and word length, all of which showed skewed distributions. In addition, a reasonable argument can be made for measuring each of these variables on a multiplicative scale. Taking logs of our dependent variable, total reading time, makes sense in particular, as this means the effects of factors in the linear model become multiplicative rather than additive. That is, if the regression predictions for the $\log$ of $t$ are a linear sum of factors, such that $\log t=\sum \beta_{i} x_{i}$, then $t$ itself can be expressed as a product of factors: $t=\prod e^{\beta_{i} x_{i}}$. This means that rather than the effects of a given predictor being additive, for example increasing reading times by $20 \mathrm{~ms}$, the effects will be multiplicative, increasing reading times by $10 \%$ for example. This makes particular sense for the differences between individuals, which are captured in the model by a set of intercepts, one for each subject. Thus rather than these intercept terms defining a fixed number of milliseconds by which a subject is faster or slower than average on each word, they define a factor by which the reading rate is slower or faster, affecting each word proportionately.

Substantial correlations were found between word length and word frequency, and 
also between launch distance, landing position and word length. The correlation of the latter three variables can be understood in terms of the fact that longer launch distances tend to result in landing positions further into a word, and given that readers prefer to land somewhere near the middle of a word, longer words tend to have greater landing positions. This correlation was removed by subtracting landing position from launch distance, so that launch distance now measured the distance from the launch site to the start of the fixated word, and also representing landing position as a proportion of word length measured from its midpoint. In addition, we included a quadratic landing position term in our set of candidate predictors, to allow the model to capture the inverted optimal viewing position effect (Vitu et al., 2001). Unfortunately, we found no effective means of removing the correlation of word frequency and word length, without undermining their predictiveness. The probabilities produced by the parser and trigram model are also, in their raw form, highly correlated. However, the breakdown of surprisal given in Equation 7.7 helps to decorrelate these two factors.

\subsubsection{Results}

We first constructed a baseline model, against which to compare our surprisal based measures. To show that these new measures are significant predictors of reading time, over and above the effects of the simpler low-level factors, our baseline contains as many of these factors as possible while retaining significance. To this end, we found a maximal set of variables which all significantly improved the model fit when introduced into a model containing the remainder. These were the log of word length, the $\log$ of transformed launch distance, the quadratic landing position, the log of unigram word probability and the $\log$ of the previous reading time. The coefficients of these terms are listed in Table 7.1.

We then took the residuals of this baseline and used them as the dependent variable in a new LME model. This allowed us to assess the contribution of the surprisal based measures after the variance due to the baseline factors had been removed, while 


\begin{tabular}{|lr|}
\hline Factor & Coefficient \\
\hline Intercept & -0.011 \\
Word Length & 0.264 \\
Launch Distance & 0.109 \\
Landing Position & 0.612 \\
Word Frequency & -0.010 \\
Reading Time of Last Word & 0.151 \\
\hline
\end{tabular}

Table 7.1: Coefficients of the baseline LME model for total reading time

\begin{tabular}{|l|rr|}
\hline Composition & SSS Coefficient & LDA Coefficient \\
\hline Additive & $.00817^{* * *}$ & $.00804^{* * *}$ \\
Multiplicative & $.00819^{* * *}$ & $.00640^{* * *}$ \\
\hline
\end{tabular}

Table 7.2: Coefficients of LME models with integrated surprisal measure (based on SSS or LDA) as factor

avoiding including the strongly correlated surprisal and word frequency variables in the same model. Collinearity effects for such variables often make the parameter estimates unreliable and can undermine significance testing.

Initially, we evaluated our integrated surprisal measures as single predictors in a linear model as in Equation 7.10. We used both the simple semantic space (SSS) and Latent Dirichlet Allocation (LDA) representations in combination with additive and multiplicative composition functions and the coefficients of the resulting surprisal measures are given in Table 7.2. All the forms of compositional representation in this case lead to a surprisal which is significant. However, we would like to know that not only is the overall measure significant, but also that each component is making a significant contribution.

To test this, we broke the integrated measure into its trigram, semantic and syntactic components as described in Section 7.2. We then entered these in turn into a model 


\begin{tabular}{|ll|lc|}
\hline Composition & Factor & SSS Coef & LDA Coef \\
\hline \multirow{2}{*}{ Additive } & $-\log \left(p_{t}\right)$ & $.00760^{* * *}$ & $.00760^{* * *}$ \\
& $-\log (\Delta)$ & $0.0381^{* * *}$ & $.00622^{* * *}$ \\
& $-\log \left(\lambda+(1-\lambda) \frac{p_{2}}{p_{1}}\right)$ & $.00953^{* * *}$ & $.00943^{* * *}$ \\
\hline \multirow{2}{*}{ Multiplicative } & $-\log (\Delta)$ & $.00760^{* * *}$ & $.00760^{* * *}$ \\
& $\log \left(\lambda+(1-\lambda) \frac{p_{2}}{p_{1}}\right)$ & $.00882^{* * *}$ & .00133 \\
\hline
\end{tabular}

Table 7.3: Coefficients of nested LME models with the components of SSS or LDA surprisal as factors; only the coefficient of the additional factor at each step is shown

and tested the significance of each. This allows us to test the contribution of each factor over and above the effect of the previously entered terms, and Table 7.3 gives the details of these tests.

All the surprisal components are significant, except the semantic and syntactic components for the multiplicative composition on LDA representations. This is roughly in line with the findings of Chapter 6 where we found that perplexity results were worse than the baseline for a language model using the same combination. As before, the sparsity of the LDA representations leads to a loss of useful information under multiplicative composition. This means that such an approach fails to capture the semantic dependencies relevant to modelling readers expectations and produces probability estimates which fail to reflect the actual structure of text. In addition, we can also see that the coefficients for each of the three components are of the same order of magnitude as the corresponding coefficient for the integrated surprisal measure (see Table 7.2), except in the case of the multiplicative LDA model. In Section 7.2, we argued that if the integrated surprisal measure balanced the contributions of its three components appropriately, we would find that the coefficients of those components should approximate the coefficient of the integrated measure on its own. The significant coefficients in Table 7.3 suggest this is indeed the case. 


\subsubsection{Discussion}

Our results show that an integrated measure of surprisal, combining trigram, semantic and syntactic dependencies, can be used to predict processing load during reading. Consolidating these factors into a single measure is preferable over a situation in which syntactic constraint is modelled in terms of surprisal (Hale, 2001) whereas semantic constraint is modelled in terms of cosine similarity (Pynte et al., 2008). Given that they are both attempting to quantify the same thing, i.e. the cognitive costs associated with the language processors expectations about upcoming input, measuring them on incommensurable scales is unappealing. Furthermore, integrating these factors into a single integrated measure simplifies their treatment and reduces the number of parameters required in modelling.

Using this integrated measure, we have evaluated our compositional representations as predictors of processing load during reading, and found results that are consistent with our language modelling and phrase similarity experiments. However, while these results both accord roughly with the conclusions of previous chapters and also support the claim that semantic composition can be modelled in terms of distributional representations, the LME method we used has a substantial drawback. That is, it cannot compare two alternative measures and provide an analysis of whether one is a significantly better predictor than the other. So, for instance, we were unable to conclude whether additive composition or multiplicative composition was better on the simple semantic space. This is related to the repeated measures nature of eye-tracking data, and this issue might be alleviated by using a different experimental paradigm.

Another criticism of the analysis carried out in this chapter is that we considered only a limited range of factors contributing to processing cost. For example, we did not consider costs relating to the introduction of discourse referents or the integration costs proposed in DLT. A more scrupulous analysis would deal with these factors too. However, the current evidence suggests that these measures may not be as wide-coverage as surprisal. 


\subsection{Conclusions}

In this chapter, we demonstrated that distributional models of semantic composition can be evaluated in terms of their ability to predict the cognitive load incurred during reading. We constructed a surprisal based measure of processing cost from the language models proposed in Chapter 6, and used this measure to predict reading times derived from an eye-tracking study. All of the semantic representations and composition functions we considered were significant predictors, with the exception multiplicative composition on LDA representations. This finding is consistent with those of previous chapters, where previous experiments have shown the deficiency of this particular combination.

In future work, it would be desirable to integrate more deeply the syntactic and semantic components of the language model to produce a more psychologically plausible model. Similarly, incorporating a wider range of factors, such as discourse and integration costs, would be appealing. In fact, Demberg and Keller (2009) propose an incremental parsing model which incorporates both prediction based surprisal costs and also locality based integration costs. 


\section{Chapter 8}

\section{Conclusions}

This chapter will summarise the research presented in previous chapters and will discuss possible future work following on from this. We will begin by reviewing the aims of this thesis before describing the contributions of this research, including the general framework and novel composition functions that were proposed, the similarity ratings that were gathered and the language model that was developed and applied to modelling reading times. Following that we will discuss the implications of our findings and identify potential weaknesses. Finally, we will outline possible future work which develops and applies the approach presented here.

\subsection{Aims of the Thesis}

The subject matter of the thesis is the question of how composition operates in distributional models of semantics. These models construct a representation for the meaning of a word from its pattern of usage, and have been used, for example, to model semantic priming (Landuaer and Dumais, 1997; Lund and Burgess, 1996). While there has been substantial research into the representation of individual words within such models, the representation of larger structures has received relatively less attention. It is widely recognised that semantic composition plays a central role in linguistic communication, allowing complex meanings to be built up for phrases and sentences through 
a process of combining and integrating the semantics of the constituents (Frege, 1884; Pinker, 1994; Partee, 1995). For example, a model of semantic priming in sentential contexts ought to take account of the fact that the effect is not simply based on a relationship between individual words, but is dependent on the combination of word meanings and their syntactic structure (Duffy et al., 1989; Morris, 1994). However, work on the composition of distributional representations to produce phrase and sentence representations has typically made unjustified assumptions (e.g., that addition is the appropriate approach: Landauer et al., 1997; Foltz et al., 1998; Coccaro and Jurafsky, 1998), or made only weak evaluations of the possible approaches (e.g., Kintsch, 2001; Widdows, 2008). Consequently, the aim of this thesis is to consider a wide range of potential composition operations, and to show that they can be evaluated on substantial quantities of natural data. Our approach has been to use a general discussion of the nature of semantic composition to derive a framework for composition in distributional models, containing existing proposals and novel approaches, and then to carry out three empirical evaluations. The first of these tests the models' predicted similarity ratings directly against human similarity judgements. The second evaluates these relations in a practical task, by exploiting them to enhance $n$-gram language models using long range semantic dependencies. Finally, the third task assesses the relevance of these dependencies to cognitive processes in reading, in terms of a model of eye-movements.

\subsection{Summary of Contributions}

In Chapter 4 we introduced a framework for considering the operation of composition in distributional models. The form of this framework was based on the discussion of the general nature of semantic composition in Section 2.3, and assumed that a compositional structure has a meaning which is a function of the constituent meanings, their syntax and also any relevant background knowledge. This formulation allowed 
us to differentiate between additive and multiplicative composition models and encompassed several existing proposals, including simple vector addition, Kintsch's (2001) model, the tensor product (Aerts and Czachor, 2004; Clark and Pulman, 2007; Widdows, 2008), and circular convolution (Widdows, 2008). Furthermore, this framework enabled us to derive a number of novel proposals. The simple multiplicative model combines the components of a pair of vector constituents by taking the product of corresponding components, and is the multiplicative equivalent of simple vector addition. The weighted additive model also generalises vector addition by weighting the contribution of each constituent by a linear factor. Finally, dilation stretches one constituent in the direction of the other to emphasise the components most salient to their combination.

In Chapter 5, we evaluated these composition functions on their ability to predict subject similarity judgements for short phrases and sentences. The experimental materials included subject-verb, adjective-noun, noun-noun and verb-object constructions and the performance of our models was measured in terms of their correlation with the subject ratings. Our experiments improve on previous approaches by being based on a substantial number of items drawn from a corpus of natural text, rather than a few hand picked examples, and by testing the significance of the model performances on ratings collected from a large number of subjects.

The candidate composition functions were applied to two distributional models: a simple semantic space based on word co-occurence and an LDA model based on the document structure of a corpus. Of these models, the best overall performance was achieved by the simple multiplicative function on the simple semantic space. In contrast, the same approach to composition on the LDA space produced much weaker results. Strong results across both spaces were attained by the weighted additive and the dilation model. Whereas circular convolution produced very poor correlations for both types of representation. Interestingly, the compositional approach to modelling phrase similarities outperformed an alternative approach based on treating the phrase 
as a single non-compositional unit.

Having evaluated these composition models in terms of their ability to predict subject similarity ratings, Chapter 6 then investigated their ability to make predictions about the semantic relations present in a corpus of news data, in terms of their contribution to probabilistic language models. By modifying the cosine measure used to produce predicted similarity ratings in Chapter 5, we derived an expression for the probability of upcoming words given their history, which took into account the semantic relatedness and also the overall frequencies of the upcoming words. Perplexity results for the resulting language models showed that the simple multiplicative model was most effective for the simple sematic space, whereas for the LDA representations, the best results were achieved with the simple additive model. In fact, the simple multiplicative model in this case actually made the perplexity worse. These results are consistent with those of the similarity rating evaluations of Chapter 5 , where the simple multiplicative and simple additive models showed a similar pattern of results on the two types of representation. Integrating these semantic probabilities with an $n$-gram model and an incremental parser yielded further perplexity reductions, demonstrating that the compositional models are effective in capturing long range semantic dependencies which are distinct from the syntactic dependencies exploited by the parser.

These results show that the compositional representations can be used to quantify the semantic relations between words and their histories in the context of predicting word sequences in language modelling. The question remained, however, whether these relations and predictions were relevant to the semantic structure perceived by language users during comprehension. In Chapter 7 we therefore investigated the use of these compositional language models in predicting the cognitive load experienced during reading. We derived a surprisal based predictor of processing load from the integrated $n$-gram, semantic, syntactic probabilities and assessed this alongside a number of other predictors in a regression model of reading times. Each of the three components of this integrated surprisal measure was found to be a significant predictor, 
except in the case of the multiplicative semantic model on the LDA space, which again is consistent with our previous findings that such a combination yields poor results.

\subsection{General Discussion}

The experiments of Chapters 5, 6 and 7 all find a similar pattern of performance with regard to the application of the simple additive and multiplicative composition functions to the simple semantic space and LDA based representations. In summary, multiplicative composition is more effective on the simple semantic space, whereas the additive function is better in the LDA model. This consistency is reassuring, in that it suggests we are probing a single well defined question with all three tasks.

However, it also demonstrates the interdependence of composition operations and the underlying semantic representation. It is perhaps obvious that the success of a particular vector composition function depends to a great extent on the structure of the representations it is applied to. In the case of the LDA representations, the weak performance of the simple multiplicative model is most easily understood as being the result of the sparsity of these vectors, which in turn is a result of the structure of the LDA model. Two contributing factors are worth drawing attention to in this regard. The first is that the contexts which LDA uses to infer its topic based representations are much wider and less specific than the word co-occurrence contexts of the simple semantic space, and consequently the semantic information captured in patterns of distribution across these contexts is blunter and can be represented as the mixture of a small number of topics. The second factor is the use of Dirichlet priors which bias the representations towards topic mixtures which are highly localised, consisting of only a few active components. This sparsity means that in the combination of two content words we need to represent the union of the topics that are associated with both constituents, and addition is a reasonable means of capturing this. Multiplication, on the other hand, of the typically disjoint vectors, results in a loss of useful information. 
In contrast, the simple semantic space vectors are generally highly distributed, with a typical target word co-occurring with many different context words. In this case, combination of two constituents is better represented by intersection of the context sets, with multiplication being an effective means of accentuating the contributions of those components that are relevant to both words' semantic content. Addition, in contrast, produces much weaker results on this space, because by including all content relevant to either word it produces something too general.

This strong dependence of the performance of these composition functions on the type of representation they are applied to suggests that, rather than develop these two components separately, it would be advantageous to integrate both into a single model from the beginning, allowing us to construct vectors which are adapted to the chosen form of composition. To some extent, the compositional language model of Chapter 6 could achieve this. As suggested in Section 6.4, vector representations could be constructed by optimising the likelihood of this model. However, this model, as it stands, is not entirely satisfactory, as it ignores syntax in order to achieve incrementality and efficiency. Nonetheless, such simple models provide a useful baseline for comparison against enhanced models which take syntactic structure into account.

Of the syntax aware composition functions, the weighted addition and dilation models produced reasonably good results for both types of representation on the phrase similarity tasks of Chapter 5. Circular convolution, on the other hand, produced the poorest results overall on these tasks. This indicates that such vector binding operations are not, by themselves, an appropriate model for semantic composition. As discussed in Section 2.2, these models are designed to mimic the syntactic process of symbol concatenation by allowing a pair of vectors to be bound together and then unbound at some later time, and can be thought of in terms of memory for hierarchical structures. However, semantic composition is distinct from the memorisation of constituents and their structure, as the experiments on sentence recall (Sachs, 1967, 1988; Begg, 1971) discussed in Section 2.3 show. Thus, it is not surprising that semantic composition 
cannot be reduced to the simple syntactic concatenation of representations.

However, neither vector binding operations nor symbolic representations are being argued to be irrelevant to modelling semantic composition. Instead, we are simply pointing out that concatenation, whether of symbols or vectors, is not by itself sufficient. These operations may have their part to play in a larger scheme for composing semantic representations, but they do not, by themselves, supply the full answer. Ultimately, we need to answer the question, raised in Section 2.3, of what sort of function of the parts composition requires. Our experiments show that the function is not simply concatenation of the constituents.

In evaluating what sort of function of the parts is required we investigated the performance of our models on the previously discussed similarity prediction and language modelling tasks in addition to the final eye-movement experiments. These latter experiments on reading times allowed us to assess the extent to which our compositional models reflect subject's incremental processing of semantic information during reading. However, to achieve this incrementality we chose to use non-syntactic composition models, specifically the simple additive and simple multiplicative models, which could be applied in linear order to the sentence words. This choice was largely motivated by the fact that standard approaches to syntactic structure are focused on the representation of complete constituents, as opposed to the partial structures required for incrementality. For example, while practical difficulties and practical difficulties slowed progress can be assigned the types $N P$ and $S$ respectively, no such simple syntactic type can be assigned to the sentence prefix practical difficulties slowed. Furthermore, a semantic representation for such a fragment cannot be constructed by recursive composition on the parse tree, because this structure is incomplete. Instead, we applied a syntax free approach, using the simple additive and multiplicative models, to the construction of semantic representations for such structures. However, this approach to composition is able, nontheless, to produce effective results, given the right choice of vector combination function for the chosen distributional model. 


\subsection{Future Work}

One of the main prospects for enhancing this work is in the handling of syntax. While we were successful in incorporating a role for syntax in a number of areas, this aspect of our models has the most potential to be developed and elaborated, because of its importance in the process of composition. The dependence of semantic composition on syntactic structure was represented explicitly in our framework, facilitating the development of syntax aware approaches, such as the weighted addition and dilation models, and these models gave competitive results on the phrase similarity task. However, their dependence on syntax was essentially based on a simplistic differential weighting of the constituents.

Furthermore, our underlying semantic representations took no account of syntactic structure at all, being based on contexts that were treated as bags-of-words. In contrast, many other distributional representations exploit the syntactic relations within contexts to enhance their performance. One approach is to use word co-occurence counts that are further broken down by the syntactic dependencies between those words (Padó and Lapata, 2007; Lin, 1998; Grefenstette, 1994). However, the dependencies available to one part of speech are often substantially different to those for a different part of speech. This means that such dependency based distributional representations produce very different vectors for distinct syntactic classes. This becomes a problem when combining two such vectors and the disjoint components have to be reconciled.

The model of Erk and Pado (2008) attempts to overcome this issue by creating vector representations not only of a target word itself, but also of its dependencies. The representation for a particular dependency is then the sum of the word vectors of the tokens that stand in that dependency relation to the target word. For example, a verb's subject dependency vector would be the sum of all the vectors for nouns that are found in the subject slot for that verb. When a noun subject and verb then compose, that noun's vector is combined with the verb's subject vector, and since this is also built from noun vectors, the two representations have comparable components. Erk 
and Pado (2008) apply this method to a word sense disambiguation task which involves finding synonyms which may be substituted for a given word in context (McCarthy and Navigli, 2007). However, this approach is not truly compositional, because it combines vectors of two different types - word representations and dependency representations with the result that its products cannot be recursively combined with other constituents higher in the tree. For example, once the subject and verb have composed there is no means to compose the result with the representation of a model or auxiliary which governs the original verb. Instead, Erk and Pado (2008) present their method as model of word meaning in context, in other words, how an individual word's meaning is modulated by the other words around it. Nonetheless, this approach probably provides a useful basis on which to explore the problem of composition further. In particular, the idea that the semantic representation of a dependent should interact with a syntactically relevant part of the head, rather than the whole head vector, seems like a promising direction for further investigation.

Rather than explore these issues here, we chose to use the simpler bag-of-words approach to context which treats all parts of speech in the same manner. This allowed us to evaluate a range of different approaches to vector combination and develop a compositional language model. However, in future work, we would ideally like to create a model in which syntax plays a sophisticated role in both the creation of the vector representations and also their composition to represent phrases and sentences. In fact, it is probable that handling the construction of representations and their composition in a holistic manner from the start will yield better results than treating them as separate issues to be reconciled later.

It is also likely that a probabilistic model that explains the distribution of words across contexts, in the mould of LDA, will provide a better opportunity to deal with these issues consistently than an ad-hoc approach, such as our simple semantic space. LDA is a generative model of the occurrence of words within documents, but it fails to deal with the internal structure, syntactic or otherwise, of those documents. Moreover, 
within this model, words are generated separately and independently, reducing the potential for modelling how meanings interact or combine. A model that addresses the issue of semantic composition will need to take account of the semantic dependencies between words and the role of syntax in these relationships.

Integrating the compositional models into a probabilistic parser may be an effective way to approach this problem. The aim would be that while the syntactic structure discovered by the parser would drive the composition of semantic representations, this semantic structure would in turn inform the comparison of alternative parses. Such a model could be evaluated in a number of ways, including the standard measures of parsing accuracy. However, because our interest in this model is as a tool to develop compositional representations, it may be more appropriate to employ semantic tasks such as predicting word and phrase similarities or modelling contextual priming effects.

Boyd-Graber and Blei (2008) integrate semantic and syntactic structure in a single model based on LDA. Their approach allows the syntactic part of the model to control the overall structure of sentences while content selection for the words within that structure is controlled by the semantic component. However, this is not a compositional model. Instead, the semantic part of the model deals with the topical vocabulary within documents and the probable transitions between topics. A compositional model would use syntactic structure to construct representations of the full meanings of sentences and their phrasal constituents, rather than just using topic representations to select the right vocabulary.

A more sophisticated approach to the role of syntax probably also requires that we move beyond viewing it simply as a relationship between content words to a perspective that incorporates representations of function words and also methods of handling complex structures, such as relative clauses and co-ordinations. Furthermore, we probably also need to develop a more sophisticated approach to handling incrementality. In Chapters 6 and 7, we produced incremental semantic interpretations by ignoring syn- 
tax. Future work ought to reconcile the way that syntax is handled with the desire for incrementality, possibly by employing a more sophisticated form of grammar, such as Tree Adjoining Grammar (TAG, Joshi et al., 1975), which has been argued to provide a closer fit to the psycholinguistic evidence concerning incrementality (Sturt and Lombardo, 2005). Moreover, the incremental parser of Demberg and Keller (2009) uses the TAG formalism to construct a unified model of prediction and integration effects on cognitive load, making it an ideal basis on which to develop a psycholinguistic model of semantic processing.

Hierarchical Hidden Markov Models (HHMM, Schuler et al., 2008) provide another grammar formalism which is directed at incrementality and psycholinguistic plausibility. $\mathrm{Wu}$ (2010) proposes an extension of this model, based on vector operations, which incorporates semantic dependencies. However, the semantic concepts employed are not the spatial representations considered here, but are instead lexical heads, clusterings of heads or logical structures. Nonetheless, the framework $\mathrm{Wu}$ (2010) proposes could be an interesting basis on which to build more research.

In addition to enhancing our models, we would also like to extend the range of their evaluation and application. One particularly interesting task would be modelling inductive inference. Deductive inference, for comparison, involves determining that a conclusion must be true given the assumptions and is the primary application of logical representations, being particularly amenable to symbolic processing. In contrast, the conclusions of inductive inferences are merely probable generalisations from the assumptions. The process by which subjects make such generalisations has often been linked to similarity relations (Heit and Rubinstein, 1994), and so may be more amenable to distributional representations. More generally, entailment (Dagan et al., 2006) and paraphrasing tasks (Dolan et al., 2004) are likely to provide appropriate applications for a model of semantics designed to quantify the similarity between pairs of phrases or sentences.

Distributional methods have also been applied to the tasks of word sense disam- 
biguation (McCarthy et al., 2004) and semantic role labelling (Fürstenau and Lapata, 2009). Moreover, these tasks involve the semantics of multiple constituents within some syntactic structure, rather than of single isolated words. As such, compositional models are likely to be relevant to their analysis. In particular, if we wish to know which training examples are most similar, and thus most informative, to a particular test construction, then composition of distributional representations may be an ideal approach to quantifying this. 


\section{Appendix A}

\section{Simple Vector and Tensor Algebra}

Formally, vectors are defined as objects within a vector space, which is itself defined in terms of the abstract relations and properties of the objects it contains. Informally, we usually think of a vector as something having magnitude and direction, such as an arrow in space. From the point of view of computation, we can always represent vectors concretely in terms of some particular basis, that is as a set of co-ordinates. Thus, a vector $\mathbf{v}$ is represented by its components $v_{i}$ in that basis.

An important relation between vectors is the dot product, defined as the sum of the products of the components:

$$
\mathbf{u} \cdot \mathbf{v}=\sum_{i} u_{i} v_{i}
$$

where the index $i$ ranges over all the components (i.e., the dimensions of the space). This allows us to define the length of vectors:

$$
|\mathbf{v}|=\sqrt{\mathbf{v} \cdot \mathbf{v}}
$$

The dot product also has a useful relation to the angle, $\theta$, between the two vectors:

$$
\mathbf{u} \cdot \mathbf{v}=|\mathbf{u}||\mathbf{v}| \cos (\theta)
$$

This implies that the dot product of any two orthogonal vectors $\left(\theta=90^{\circ}\right)$ is zero. Equations A.1 - A.3 allow us to calculate the cosine of the angle as:

$$
\cos (\theta)=\frac{\sum_{i} u_{i} v_{i}}{\sqrt{\sum_{i} u_{i} u_{i}} \sqrt{\sum_{i} v_{i} v_{i}}}
$$


The two most basic transformations which operate on vectors are addition and multiplication by a scalar:

$$
\begin{gathered}
\mathbf{p}=\mathbf{u}+\mathbf{v} \\
p_{i}=u_{i}+v_{i} \\
\mathbf{q}=s \mathbf{v} \\
q_{i}=s v_{i}
\end{gathered}
$$

Any vector $\mathbf{v}$, can be expressed in terms of a component, $\mathbf{v}_{\|}$, parallel to and a component, $\mathbf{v}_{\perp}$, orthogonal to a second vector, $\mathbf{u}$.

$$
\mathbf{v}=\mathbf{v}_{\|}+\mathbf{v}_{\perp}
$$

Taking the dot product of $\mathbf{u}$ on both sides of this equation yields:

$$
\mathbf{v} \cdot \mathbf{u}=\left|\mathbf{v}_{\|}\right||\mathbf{u}| \operatorname{Cos}\left(0^{\circ}\right)+\left|\mathbf{v}_{\perp}\right||\mathbf{u}| \operatorname{Cos}\left(90^{\circ}\right)
$$

Since $\operatorname{Cos}\left(0^{\circ}\right)=1$ and $\operatorname{Cos}\left(90^{\circ}\right)=0$, this implies:

$$
\left|\mathbf{v}_{\|}\right|=\frac{\mathbf{v} \cdot \mathbf{u}}{|\mathbf{u}|}
$$

Thus, we can construct $\mathbf{v}_{\|}$by normalizing $\mathbf{u}$ to give a unit vector which points in the right direction, and then multiplying by the right magnitude $\left|\mathbf{v}_{\|}\right|$.

$$
\mathbf{v}_{\|}=\frac{\mathbf{v} \cdot \mathbf{u}}{|\mathbf{u}|} \frac{\mathbf{u}}{|\mathbf{u}|}=\frac{\mathbf{v} \cdot \mathbf{u}}{\mathbf{u} \cdot \mathbf{u}} \mathbf{u}
$$

The orthogonal component, $\mathbf{v}_{\perp}$, can then be calculated from the fact that the two components must combine to give $\mathbf{v}$.

$$
\mathbf{v}_{\perp}=\mathbf{v}-\frac{\mathbf{v} \cdot \mathbf{u}}{\mathbf{u} \cdot \mathbf{u}} \mathbf{u}
$$

Another important set of transformations of vectors are the linear transformations induced by matrices.

$$
\mathbf{w}=\mathbf{M v}
$$




$$
w_{i}=\sum_{j} M_{i j} v_{j}
$$

A matrix, $\mathbf{M}$, is represented by an array of values, $M_{i j}$, indexed by a pair of indices, $i$ and $j$. The element $M_{i j}$ can be thought of as determining how much the $j$ th component of the original vector, $\mathbf{v}$, contributes to the $i$ th component of the transformed vector, $\mathbf{w}$.

Alternatively, we can think of $\mathbf{M}$ in terms of its polar decomposition into a matrix, $\mathbf{D}$, of dilations, and a matrix, $\mathbf{R}$, of rotations.

$$
\mathbf{M}=\mathbf{D R}
$$

The action of $\mathbf{D}$ on a vector is to stretch it by various amounts in various directions, and the action of $\mathbf{R}$ is to rotate it around various axes, without changing its length. The directions in which a matrix dilates vectors without changing their direction are known as eigenvectors, and the amounts by which they are stretched are known as eigenvalues.

The tensor product, $\otimes$, takes a pair of vectors and combines them to form a higher dimensional vector.

$$
\mathbf{t}=\mathbf{u} \otimes \mathbf{v}
$$

In particular, if $\mathbf{u}$ and $\mathbf{v}$ have dimension $n$, then $\mathbf{t}$ has dimension $n^{2}$. The components of $\mathbf{t}$ are all the pairwise products of the components of $\mathbf{u}$ and $\mathbf{v}$.

$$
t_{i j}=u_{i} v_{j}
$$

It is possible to project such a product of vectors down onto a vector of the same dimension using a rank 3 tensor.

$$
\mathbf{r}=\mathbf{C u v}
$$

Again this is a linear transformation. The tensor $\mathbf{C}$ has three indices, corresponding to the three vectors $\mathbf{r}, \mathbf{u}$ and $\mathbf{v}$.

$$
r_{i}=\sum_{j k} C_{i j k} u_{k} v_{j}
$$

A simple example of such a rank 3 tensor would be one in which $C_{i j k}=1$ when $i=$ $j=k$ and 0 otherwise, which yields:

$$
r_{i}=u_{i} v_{i}
$$


which can be also written as:

$$
\mathbf{r}=\mathbf{u} \odot \mathbf{v}
$$

A more complex example is the tensor, $\mathbf{C}$, with components $C_{i j k}=1$ when $k=(i-j) \bmod _{n}$ and 0 otherwise.

$$
r_{i}=\sum_{j} u_{j} v_{(i-j) \bmod _{n}}
$$

also written as:

$$
\mathbf{r}=\mathbf{u} \circledast \mathbf{v}
$$

Multiplying a single vector, $\mathbf{u}$, by a rank 3 tensor, $\mathbf{C}$, produces a matrix, $\mathbf{U}$.

$$
\begin{gathered}
\mathbf{U}=\mathbf{C u} \\
U_{i j}=\sum_{k} C_{i j k} u_{k}
\end{gathered}
$$

Multiplication of $\mathbf{v}$ by this matrix, $\mathbf{U}$, then results in the same vector, $\mathbf{r}$, produced by the product Cuv:

$$
\begin{gathered}
\mathbf{U v}=\mathbf{C u v}=\mathbf{r} \\
\sum_{j} U_{i j} v_{j}=\sum_{j} \sum_{k} C_{i j k} u_{k} v_{j}=r_{i}
\end{gathered}
$$

It is also possible to define higher order tensors, such as a rank 4 tensor which acts on three vectors:

$$
\begin{gathered}
\mathbf{x}=\text { Duvy } \\
x_{i}=\sum_{j k l} D_{i j k l} u_{l} v_{k} y_{j}
\end{gathered}
$$




\section{Appendix B}

\section{Instructions for Experiment 1}

In this experiment you will be shown a pair of sentences. Your task is to use your first impression of the meaning of each sentence to judge how similar they are. You will make this judgement by choosing a rating from 1 (not very similar) to 7 (very similar).

For example, if you were asked to make the following comparison:
(1) a. The employees were canned.
b. The employees were tinned.

you would give this a low similarity rating (e.g. 1 or 2). In this case, 'canned' is being used to mean 'fired'. On the other hand, if you were given the following comparison:
(2) a. The sardines were canned.
b. The sardines were tinned.

you would probably choose a high similarity rating (e.g. 6 or 7).

Sometimes the two sentences will have meanings that are moderately different though still have much in common. For instance, in this comparison:
(3) a. The helicopter hovered.
b. The helicopter lingered.

you would choose a middling rating (e.g. 3, 4 or 5) if you felt that the meanings of the 
two sentences were fairly similar but also reasonably different.

There are no 'correct' answers, so whatever choice seems appropriate to you is a valid response. Simply try to rate how similar the meanings of the two sentences are. Base your judgement on your first impression of what each sentence means. The whole experiment should take only about 10 minutes. 


\section{Appendix C}

Materials for Experiment 1 
Table C.1: Materials for eliciting similarity judgments on subject-verb combinations.

The discussion strayed. - The discussion digressed., The child strayed. - The child digressed., Her eyes strayed. - Her eyes roamed., Her thoughts strayed. - Her thoughts roamed., His mind reeled. - His mind whirled., The industry reeled. - The industry whirled., The man reeled. - The man staggered., His head reeled. - His head staggered., Optimism rebounded. - Optimism rallied., The ball rebounded. - The ball rallied., Her shot rebounded. - Her shot ricocheted., The shares rebounded. - The shares ricocheted., The screen flickered. - The screen flicked., Hope flickered. - Hope flicked., Her interest flickered. - Her interest wavered., Her tongue flickered. - Her tongue wavered., Its value slumped. - Its value declined., The man slumped. - The man declined., His shoulders slumped. - His shoulders slouched., Sales slumped. - Sales slouched., His head bowed. - His head stooped., The government bowed. - The government stooped., The company bowed. - The company submitted., The butler bowed. - The butler submitted., Her voice throbbed. - Her voice shuddered., Her head throbbed. - Her head shuddered., The vein throbbed. - The vein pulsed., His body throbbed. - His body pulsed., The argument flared. - The argument erupted., The cigarette flared. - The cigarette erupted., Her eyes flared. Her eyes flamed., The row flared. - The row flamed., Her temper erupted. - Her temper flared., The storm erupted. - The storm flared., The fountain erupted. - The fountain burst., The conflict erupted. - The conflict burst., Her eye recoiled. - Her eye flinched., Her heart recoiled. - Her heart flinched., Her rifle recoiled. - Her rifle kicked., His hand recoiled. His hand kicked., The gun boomed. - The gun thundered., Sales boomed. - Sales thundered., Exports boomed. - Exports prospered., The noise boomed. - The noise prospered., Her determination wavered. - Her determination faltered., Her concentration wavered. Her concentration faltered., Opinion wavered. - Opinion fluctuated., His courage wavered. - His courage fluctuated., Her fear subsided. - Her fear lessened., The flood subsided. The flood lessened., The island subsided. - The island sank., The symptoms subsided. The symptoms sank., The machine chattered. - The machine clicked., The child chattered. - The child clicked., The girl chattered. - The girl gabbled., Her teeth chattered. - Her teeth gabbled., His face glowed. - His face beamed., The cigar glowed. - The cigar beamed., The fire glowed. - The fire burned., His skin glowed. - His skin burned. 
Table C.2: Materials for eliciting similarity judgments on subject-verb combinations continued.

Her thoughts strayed. - Her thoughts digressed., Her eyes strayed. - Her eyes digressed., The child strayed. - The child roamed., The discussion strayed. - The discussion roamed., His head reeled. - His head whirled., The man reeled. - The man whirled., The industry reeled. - The industry staggered., His mind reeled. - His mind staggered., The shares rebounded. - The shares rallied., Her shot rebounded. - Her shot rallied., The ball rebounded. - The ball ricocheted., Optimism rebounded. - Optimism ricocheted., Her tongue flickered. - Her tongue flicked., Her interest flickered. - Her interest flicked., Hope flickered. - Hope wavered., The screen flickered. - The screen wavered., Sales slumped. - Sales declined., His shoulders slumped. - His shoulders declined., The man slumped. - The man slouched., Its value slumped. - Its value slouched., The butler bowed. - The butler stooped., The company bowed. - The company stooped., The government bowed. - The government submitted., His head bowed. - His head submitted., His body throbbed. - His body shuddered., The vein throbbed. - The vein shuddered., Her head throbbed. - Her head pulsed., Her voice throbbed. - Her voice pulsed., The row flared. - The row erupted., Her eyes flared. - Her eyes erupted., The cigarette flared. - The cigarette flamed., The argument flared. - The argument flamed., The conflict erupted. - The conflict flared., The fountain erupted. - The fountain flared., The storm erupted. - The storm burst., Her temper erupted. - Her temper burst., His hand recoiled. - His hand flinched., Her rifle recoiled. - Her rifle flinched., Her heart recoiled. - Her heart kicked., Her eye recoiled. - Her eye kicked., The noise boomed. - The noise thundered., Exports boomed. - Exports thundered., Sales boomed. - Sales prospered., The gun boomed. - The gun prospered., His courage wavered. - His courage faltered., Opinion wavered. - Opinion faltered., Her concentration wavered. - Her concentration fluctuated., Her determination wavered. - Her determination fluctuated., The symptoms subsided. - The symptoms lessened., The island subsided. The island lessened., The flood subsided. - The flood sank., Her fear subsided. - Her fear sank., Her teeth chattered. - Her teeth clicked., The girl chattered. - The girl clicked., The child chattered. - The child gabbled., The machine chattered. - The machine gabbled., His skin glowed. - His skin beamed., The fire glowed. - The fire beamed., The cigar glowed. The cigar burned., His face glowed. - His face burned. 


\section{Appendix D}

\section{Instructions for Experiment 2}

In this experiment you will be shown a pair of noun phrases. Your task is to judge how similar the two phrases are. You will make this judgement by choosing a rating from 1 (not very similar) to 7 (very similar). The focus is on the similarity of the concepts named by the phrases, not any association between the two phrases.

For example, if you were asked to make the following comparison:
(1) a. professional advice
b. expert opinion

you would give this a high similarity rating (e.g. 6 or 7). Both these phrases concern guidance or instruction from a knowledgeable person and so have highly similar meanings. On the other hand, if you were given the following comparison:
(2)
a. social worker
b. wide range

you would probably choose a low similarity rating (e.g. 1 or 2 ), since one is an occupation and the other is a magnitude. Likewise, for this comparison:
(3) a. increasing taxation
b. public protest 
you would also choose a low similarity rating (e.g. 1 or 2 ), since they are different things, even though they might be associated, in that the first could lead to the second. Of course, associated phrases may also be similar.

Sometimes the two phrases will have meanings that are moderately different though still have much in common. For instance, in this comparison:
(4)
a. human behaviour
b. social activity

you would choose a middling rating (e.g., 3, 4 or 5) if you felt that the meanings of the two phrases were reasonably different but also had some similarities. For instance both involve the interactions of people, although the two phrases also invoke other distinct concepts.

There are no 'correct' answers, so whatever choice seems appropriate to you is a valid response. Simply try to rate how similar the meanings of the two phrases are. Base your judgment on your first impression of what each phrase means. The whole experiment should take only about 10 minutes.

Remember:

- Rate the similarity of the phrases not their association.

- Base your judgment on your first impression of what each phrase means.

- There are no correct answers.

At the start of the experiment you will be given a few examples to practice on. 


\section{Appendix E}

\section{Materials for Experiment 2}

Our experimental stimuli for adjective-noun, noun-noun, and verb-object combinations are shown in Tables E.1-E.3, respectively.

Table E.1: Materials for eliciting similarity judgments on adjective-noun combinations.

High

American country-European state, industrial area-whole country, vast amountlarge quantity, new body-whole system, small house-little room, early eveningprevious day, special circumstance-particular case, black hair-dark eye, new information-further evidence, economic development-rural community, economic problem-practical difficulty, new law-public building, general principle-basic rule, central authority-local office, older man-elderly woman, high price-low cost, different kind-various form, old person-elderly lady, better job-good place, new lifeearly age, certain circumstance-economic condition, earlier work-early stage, federal assembly-national government, effective way-efficient use, social activitypolitical action, similar result-good effect, major issue-social event, different partnorthern region, important part-significant role, new situation-present position, right hand-left arm, general level-high point, large number-great majority, long period-short time, hot weather-cold air, modern language-new technology 


Medium
new life-modern language, good place-high point, social activity-economic con-
dition, different part-various form, better job-good effect, old person-right hand,
local office-new technology, high price-short time, social event-low cost, early
stage-long period, efficient use-significant role, national government-cold air, large
number-vast amount, economic problem-new situation, new information-general
level, small house-important part, European state-present position, political action-
economic development, large quantity-great majority, dark eye-left arm, northern
region-industrial area, little room-similar result, major issue-American country,
hot weather-further evidence, new law-basic rule, certain circumstance-particular
case, older man-new body, previous day-early age, earlier work-early evening,
public building-central authority, elderly woman-black hair, different kind-whole
system, effective way-practical difficulty, whole country-general principle, rural
community-federal assembly, special circumstance-elderly lady




\section{Low}

new situation-different kind, effective way-important part, general level-federal assembly, central authority-political action, major issue-earlier work, older mangreat majority, large number-certain circumstance, general principle-present position, similar result-basic rule, northern region-early age, left arm-elderly woman, hot weather-elderly lady, new law-modern language, previous day-long period, whole country-different part, social activity-whole system, new technologypublic building, high point-particular case, social event-special circumstance, new body-significant role, early evening-good effect, black hair-right hand, practical difficulty-cold air, short time-rural community, new life-economic development, small house-old person, local office-industrial area, national governmentnew information, efficient use-little room, various form-European state, better jobeconomic problem, economic condition-American country, early stage-dark eye, large quantity-good place, vast amount-high price, further evidence-low cost 
Table E.2: Materials for eliciting similarity judgments on noun-noun combinations.

\begin{tabular}{|l|}
\hline High \\
development plan-action programme, telephone number-phone call, marketing \\
director-assistant manager, support group-computer system, training programme- \\
education course, training college-education officer, planning committee-education \\
authority, oil industry-computer company, health service-community care, \\
wage increase-tax rate, environment secretary-defence minister, office worker- \\
health minister, tv set-television programme, party official-government leader, \\
state control-government intervention, tax charge-interest rate, news agency- \\
intelligence service, service department-personnel manager, research work- \\
development project, company director-assistant secretary, labour cost-capital mar- \\
ket, league match-football club, world economy-market leader, state benefit- \\
housing department, town council-city centre, party leader-opposition member, \\
management structure-datum system, kitchen door-bedroom window, committee \\
meeting-board member, town hall-county council, research contract-future de- \\
velopment, railway station-bus company, care plan-business unit, study group- \\
management skill, tax credit-family allowance, security policy-housing benefit \\
\hline
\end{tabular}




Medium
state control-town council, party official-opposition member, intelligence service-
bus company, state benefit-county council, interest rate-business unit, government
intervention-party leader, research work-city centre, capital market-future devel-
opment, football club-town hall, market leader-board member, tv set-bedroom
window, labour cost-housing benefit, care plan-action programme, management
structure-computer system, datum system-support group, study group-computer
company, research contract-training programme, security policy-defence minister,
family allowance-tax rate, tax credit-wage increase, management skill-planning
committee, committee meeting-phone call, railway station-oil industry, kitchen
door-office worker, education authority-service department, development plan-
television programme, community care-tax charge, assistant manager-company
director, marketing director-personnel manager, health service-assistant secre-
tary, education officer-development project, education course-housing department,
health minister-government leader, telephone number-league match, environment
secretary-news agency, training college-world economy




\section{Low}

development project-care plan, television programme-research contract, government leader-security policy, tax charge-datum system, news agency-study group, world economy-management structure, assistant secretary-committee meeting, company director-tax credit, league match-family allowance, service departmentrailway station, housing department-kitchen door, personnel manager-management skill, bus company-health service, city centre-community care, business unitdevelopment plan, town hall-education course, future development-telephone number, party leader-environment secretary, town council-education authority, board member-assistant manager, bedroom window-education officer, county councilmarketing director, opposition member-health minister, housing benefit-training college, action programme-tv set, support group-interest rate, tax rate-market leader, training programme-research work, defence minister-government intervention, office worker-party official, computer company-intelligence service, computer system-state control, oil industry-capital market, planning committeefootball club, phone call-state benefit, wage increase-labour cost 
Table E.3: Materials for eliciting similarity judgments on verb-object combinations.

\begin{tabular}{|l|}
\hline High \\
produce effect-achieve result, require attention-need treatment, present problem- \\
face difficulty, leave company-join party, satisfy demand-meet requirement, use \\
power-exercise influence, shut door-close eye, sell property-buy land, reduce \\
amount-increase number, send message-receive letter, suffer loss-cause injury, \\
use test-pass time, write book-read word, start work-begin career, reach level- \\
achieve end, stress importance-emphasise need, use method-develop technique, \\
hold meeting-attend conference, use knowledge-acquire skill, win match-play \\
game, like people-ask man, follow road-cross line, help people-encourage child, \\
pose problem-address question, raise head-lift hand, pay price-cut cost, leave \\
house-buy home, wave hand-stretch arm, discuss issue-consider matter, provide \\
help-offer support, win battle-fight war, remember name-hear word, set example- \\
provide system, provide datum-collect information, pour tea-drink water, share \\
interest-express view
\end{tabular}




\section{Medium}

write book-hear word, address question-raise head, read word-remember name, follow road-set example, use method-drink water, hold meeting-lift hand, win match-fight war, play game-win battle, start work-wave hand, achieve end-express view, develop technique-provide help, attend conference-share interest, provide system-use power, cut cost-reduce amount, buy home-sell property, consider matter-produce effect, leave house-buy land, pay price-require attention, collect information-receive letter, offer support-need treatment, discuss issue-present problem, stretch arm-close eye, pour tea-join party, provide datum-shut door, face difficulty-pose problem, achieve result-reach level, exercise influence-use knowledge, satisfy demand-emphasise need, send message-ask man, use test-acquire skill, meet requirement-help people, leave company-encourage child, pass timecross line, suffer loss-begin career, increase number-like people, cause injurystress importance 


\section{Low}

use knowledge-provide system, pose problem-consider matter, encourage childleave house, reach level-provide datum, ask man-stretch arm, acquire skill-buy home, stress importance-cut cost, begin career-pay price, cross line-offer support, help people-discuss issue, like people-collect information, emphasise need-pour tea, drink water-use test, remember name-pass time, share interest-exercise influence, hear word-send message, wave hand-leave company, fight war-increase number, provide help-satisfy demand, raise head-cause injury, lift hand-achieve result, set example-face difficulty, express view-suffer loss, win battle-meet requirement, buy land-write book, receive letter-read word, produce effect-start work, present problem-address question, use power-develop technique, sell property-hold meeting, shut door-follow road, join party-play game, close eye-achieve end, reduce amount-win match, need treatment-use method, require attention-attend conference 


\section{Bibliography}

Aerts, D. and Czachor, M. (2004). Quantum aspects of semantic analysis and symbolic artificial intelligence. Journal of Physics A-Mathematical and General, 37:L123L32.

Altmann, G. T. M. and Kamide, Y. (1999). Incremental interpretation at verbs: Restricting the domain of subsequent reference. Cognition, 73:247-264.

Anderson, J. R. (1993). Rules of the Mind. Erlbaum, Hillsdale, NJ.

Baldwin, T., Bannard, C., Tanaka, T., and Widdows, D. (2003). An empirical model of multiword expression decomposability. In Proceedings of the ACL 2003 workshop on Multiword expressions, pages 89-96, Morristown, NJ, USA. Association for Computational Linguistics.

Bannard, C., Baldwin, T., and Lascarides, A. (2003). A statistical approach to the semantics of verb-particles. In Proceedings of the ACL 2003 workshop on Multiword expressions, pages 65-72, Morristown, NJ, USA. Association for Computational Linguistics.

Barzilay, R. and Lee, L. (2003). Learning to paraphrase: An unsupervised approach using multiple-sequence alignment. In Proceedings of the Human Language Technology Conference and Annual Meeting of the North American Chapter of the Association for Computational Linguistics, pages 16-23, Edmonton, AL. 
Begg, I. (1971). Recognition memory for sentence meaning and wording. Journal of Verbal Learning and Behaviour, 10:176-181.

Bellegarda, J. R. (2000). Exploiting latent semantic information in statistical language modeling. Proceedings of the IEEE, 88(8):1279-1296.

Bellegarda, J. R. (2004). Statistical language model adaptation: review and perspectives. Speech Communication, 42(1):93-108.

Bengio, Y., Ducharme, R., Vincent, P., and Janvin, C. (2003). A neural probabilistic language model. Journal of Machine Learning Research, 3:1137-1155.

Bever, T. G. (1970). The cognitive basis for linguistic strutures. In Hayes, J. R., editor, Cognition and the Development of Language, pages 279-362. Wiley, New York.

Blackburn, P. and Bos, J. (2005). Representation and Inference for Natural Language: A First Course in Computational Semantics. Stanford: CSLI Press, Seattle, WA.

Blei, D. M., Ng, A. Y., and Jordan, M. I. (2003). Latent dirichlet allocation. Journal of Machine Learning Research, 3:993-1022.

Booth, T. L. (1969). Probabilistic representation of formal languages. In Proceedings of the 10th Annual Symposium on Switching and Automata Theory, pages 74-81, Washington, DC, USA. IEEE Computer Society.

Boyd-Graber, J. L. and Blei, D. M. (2008). Syntactic topic models. In Koller, D., Schuurmans, D., Bengio, Y., and Bottou, L., editors, NIPS, pages 185-192. MIT Press.

Briscoe, E. and Carroll, J. (2002). Robust accurate statistical annotation of general text. In Third International Conference on Language Resources and Evaluation (LREC 2002), pages 1499-1504, Las Palmas, Canary Islands. 
Brysbaert, M. and Vitu, F. (1998). Word skipping: Implications for theories of eye movement control in reading. In Underwood, G., editor, Eye Guidance in Reading and Scene Perception, pages 125-147. Elsevier, Oxford.

Bullinaria, J. and Levy, J. (2007). Extracting semantic representations from word cooccurrence statistics: A computational study. Behavior Research Methods, 39:510526.

Burgess, C. and Lund, K. (1997). Representing abstract words and emotional connotation in high-dimensional memory space. In Shafto, M. G. and Langley, P., editors, Proceedings of the nineteenth annual conference of the cognitive science society, pages 61-66, Mahwah, NJ. Lawrence Erlbaum Associates.

Burgess, C. and Lund, K. (1998). Modeling cerebral asymmetries in high-dimensional space. In Beeman, M. and Chiarello, C., editors, Right hemisphere language comprehension: Perspectives from cognitive neuroscience, pages 215-244. Lawrence Erlbaum Associates, Mahwah, NJ.

Campbell, F. W. and Wurtz, R. H. (1978). Saccadic omission: why we do not see a grey-out during a saccadic eye movement. Vision Research, 18(10):1297-1303.

Carpenter, P. A. and Just, M. A. (1983). What your eyes do while your mind is reading. In Rayner, K., editor, Eye movements in reading: Perceptual and language processes, pages 275-307. Academic Press, New York.

Cattell, J. M. (1886). The time taken up by cerebral operations. Mind, 11(43):377-392.

Charniak, E. (2001). Immediate-head parsing for language models. In Proceedings of 35th Annual Meeting of the Association for Computational Linguistics and 8th Conference of the European Chapter of the Association for Computational Linguistics, pages 116-123, Toulouse, France. 
Chelba, C. and Jelinek, F. (1998). Exploiting syntactic structure for language modeling. In Proceedings of the 17th International Conference on Computational Linguistics and 36th Annual Meeting of the Association for Computational Linguistics, pages 225-231, Montréal, Canada.

Chen, S. F. and Goodman, J. (1999). An empirical study of smoothing techniques for language modeling. Computer Speech \& Language, 13(4):359-393.

Chomsky, N. (2000). New Horizons in the Study of Language and Mind. Cambridge University Press, Cambridge, UK.

Clark, S., Coecke, B., and Sadrzadeh, M. (2008). A compositional distributional model of meaning. In Proceedings of the Second Symposium on Quantum Interaction (QI2008), pages 133-140, Oxford, UK. College Publications.

Clark, S. and Pulman, S. (2007). Combining symbolic and distributional models of meaning. In Proceedings of the AAAI Spring Symposium on Quantum Interaction, Stanford, CA, 2007, pages 52-55.

Clarke, D., Lutz, R., and Weir, D. (2010). Semantic composition with quotient algebras. In Proceedings of the 2010 Workshop on GEometrical Models of Natural Language Semantics, pages 38-44, Uppsala, Sweden. Association for Computational Linguistics.

Coccaro, N. and Jurafsky, D. (1998). Towards better integration of semantic predictors in satistical language modeling. In Proceedings of the 5th International Conference on Spoken Language Processing, pages 2403-2406, Sydney, Australia.

Cohen, J. and Cohen, P. (1983). Applied Multiple Regression/Correlation Analysis for the Behavioral Sciences. Hillsdale, NJ: Erlbaum.

Collins, A. M. and Quillian, M. R. (1969). Retrieval time from semantic memory. Journal of Verbal Learning and Verbal Behavior, (8):240-248. 
Cooper, R. M. (1974). The control of eye fixation by the meaning of spoken language: A new methodology for the real-time investigation of speech perception, memory, and language processing. Cognitive Psychology, 6(1):84-107.

Curran, J. R. (2003). From Distributional to Semantic Similarity. PhD thesis, University of Edinburgh.

Dagan, I., Glickman, O., and Magnini, B. (2006). The pascal recognising textual entailment challenge. In Quionero-Candela, J., Dagan, I., Magnini, B., and d'Alch Buc, F., editors, Machine Learning Challenges, volume 3944 of Lecture Notes in Computer Science, pages 177-190. Springer.

Davidson, D. (1967). Truth and meaning. Synthese, 17:304-323.

Deerwester, S. C., Dumais, S. T., Landauer, T. K., Furnas, G. W., and Harshman, R. A. (1990). Indexing by latent semantic analysis. Journal of the American Society of Information Science, 41(6):391-407.

Demberg, V. and Keller, F. (2008). Data from eye-tracking corpora as evidence for theories of syntactic processing complexity. Cognition, 101(2):193-210.

Demberg, V. and Keller, F. (2009). A computational model of prediction in human parsing: Unifying locality and surprisal effects. In Proceedings of the 31st meeting of the Cognitive Science Society, pages 1888-1893, Austin, TX. Cognitive Science Society.

Deng, Y. and Khundanpur, S. (2003). Latent semantic information in maximum entropy language models for conversational speech recognition. In Proceedings of the 2003 Conference of the North American Chapter of the Association for Computational Linguistics on Human Language Technology, pages 56-63, Morristown, NJ, USA. Association for Computational Linguistics. 
Denhire, G. and Lemaire, B. (2004). A computational model of children's semantic memory. In Proceedings of the 26th Annual Meeting of the Cognitive Science Society, pages 297-302, Mahwah, NJ. Lawrence Erlbaum Associates.

Dolan, B., Quirk, C., and Brockett, C. (2004). Unsupervised construction of large paraphrase corpora: exploiting massively parallel news sources. In COLING '04: Proceedings of the 20th international conference on Computational Linguistics, page 350, Morristown, NJ, USA. Association for Computational Linguistics.

Doumas, L. A. A. and Hummel, J. E. (2005). Modeling human mental representations: What works and what doesn't and why. In Holyoak, K. J. and Morrison, R. G., editors, The Cambridge Handbook of Thinking and Reasoning, pages 73-91. Cambridge University Press, Cambridge, UK.

Duffy, S. A., Henderson, J. M., and Morris, R. K. (1989). Semantic facilitation of lexical access during sentence processing. Journal of Experimental Psychology: Learning, Memory, and Cognition, 15:791-801.

Ehrlich, K. and Rayner, K. (1983). Pronoun assignment and semantic integration during reading: Eyemovements and immediacy of processing. Journal of verbal learning and verbal behaviour, 22:75-87.

Ehrlich, S. F. and Rayner, K. (1981). Contextual effects on word perception and eye movements during reading. Journal of Verbal Learning and Verbal Behavior, 20(6):641-655.

Elman, J. L. (1991). Distributed representations, simple recurrent networks, and grammatical structure. Machine Learning, 7:195-224.

Erk, K. and Pado, S. (2008). A structured vector space model for word meaning in context. In Proceedings of EMNLP 2008. 
Falkenhainer, B., Forbus, K. D., and Gentner, D. (1989). The structure-mapping engine: algorithm and examples. Artificial Intelligence, 41(1):1-63.

Fellbaum, C., editor (1998). WordNet: An Electronic Lexical Database (Language, Speech, and Communication). The MIT Press.

Finkelstein, L., Gabrilovich, E., Matias, Y., Rivlin, E., Solan, Z., Wolfman, G., and Ruppin, E. (2002). Placing search in context: The concept revisited. ACM Transactions on Information Systems, 20(1):116-131.

Firth, J. R. (1957). A synopsis of linguistic theory 1930-1955. In Studies in Linguistic Analysis, pages 1-32. Philological Society, Oxford.

Fodor, J. and Lepore, E. (2002). The Compositionality Papers. Clarendon Press, Oxford.

Fodor, J. and Pylyshyn, Z. (1988). Connectionism and cognitive architecture: A critical analysis. Cognition, 28:3-71.

Foltz, P., Kintsch, W., and Landauer, T. (1998). The measurement of textual coherence with latent semantic analysis. Discourse Process, 15:285-307.

Frank, S., Koppen, M., Noordman, L., and Vonk, W. (2007). World knowledge in computational models of discourse comprehension. Discourse Processes. In press.

Frank, S. L. (2009). Surprisal-based comparison between a symbolic and a connectionist model of sentence processing. In Proceedings of the 31st Annual Conference of the Cognitive Science Society, pages 139-1144, Austin, TX.

Frege, G. (1884). Die Grundlagen der Arithmetik. W. Koebner, Breslau.

Furbach, U., Glöckner, I., and und Björn Pelzer, H. H. (2010). Logic-based question answering. Künstliche Intelligenz, 24(1):51-55. 
Fürstenau, H. and Lapata, M. (2009). Semi-supervised semantic role labeling. In EACL '09: Proceedings of the 12th Conference of the European Chapter of the Association for Computational Linguistics, pages 220-228, Morristown, NJ, USA. Association for Computational Linguistics.

Garrod, S. C. and Sanford, A. J. (1994). Resolving sentences in discourse context: How discourse representation affects language understanding. In Gernsbacher, M. A., editor, Handbook of Psycholinguistics, pages 675-698. Academic Press, San Diego.

Ge, R. and Mooney, R. J. (2009). Learning a compositional semantic parser using an existing syntactic parser. In Proceedings of the Joint Conference of the 47th Annual Meeting of the ACL and the 4th International Joint Conference on Natural Language Processing of the AFNLP, pages 611-619, Morristown, NJ. Association for Computational Linguistics.

Gibson, E. (2000). Dependency locality theory: A distance-dased theory of linguistic complexity. In Marantz, A., Miyashita, Y., and O’Neil, W., editors, Image, Language, Brain: Papers from the First Mind Articulation Project Symposium, pages 95-126. MIT Press, Cambridge, MA.

Gildea, D. and Hofmann, T. (1999). Topic-based language models using EM. In Proceedings of the 6th European Conference on Speech Communiation and Technology, pages 2167-2170, Budapest, Hungary.

Golub, G. H., Luk, F. T., and Overton, M. L. (1981). A block lanczoz method for computing the singular values and corresponding singular vectors of a matrix. $A C M$ Transactions on Mathematical Software, 7:149-169.

Good, I. (1953). The population frequencies of species and the estimation of population parameters. Biometrika, 40:237-264. 
Grefenstette, G. (1994). Explorations in Automatic Thesaurus Discovery. Kluwer Academic Publishers, Norwell, MA, USA.

Griffiths, T. L., Steyvers, M., and Tenenbaum, J. B. (2007). Topics in semantic representation. Psychological Review, 114(2):211-244.

Hahn, U., Chater, N., and Richardson, L. B. (2003). Similarity as transformation. Cognition, 87(1):1 - 32 .

Hale, J. (2001). A probabilistic Earley parser as a psycholinguistic model. In Proceedings of the 2nd Conference of the North American Chapter of the Association, volume 2, pages 159-166, Pittsburgh, PA. Association for Computational Linguistics.

Harris, Z. S. (1954). Distributional structure. Word, 10:146-162.

Haviland, S. E. and Clark, H. H. (1974). Whats new? acquiring new information as a process in comprehension. Journal of verbal learning and behaviour, 13:512-521.

Heit, E. and Rubinstein, J. (1994). Similarity and property effects in inductive reasoning. Journal of Experimental Psychology: Learning, Memory, and Cognition, 20:411-422.

Hinton, G. E. (1990). Mapping part-whole hierarchies into connectionist networks. Artif. Intell., 46(1-2):47-75.

Hofmann, T. (2001). Unsupervised learning by probabilistic latent semantic analysis. Machine Learning, 42(1-2):177-196.

Holyoak, K. J. and Hummel, J. E. (2000). The proper treatment of symbols in a connectionist architecture. In Dietrich, E. and Markman, A., editors, Cognitive Dynamics: Conceptual change in humans and machines., pages 229-264. MIT Press, Cambridge, MA. 
Hopcroft, J. E. and Ullman, J. D. (1979). Introduction to Automata Theory, Languages, and Computation. Addison-Wesley Publishing Company.

Inhoff, A. and Rayner, K. (1986). Parafoveal word processing during eye fixations in reading: Effects of word frequency. Perception and Psychophysics, 40:431-439.

Jiang, J. J. and Conrath, D. W. (1997). Semantic similarity based on corpus statistics and lexical taxonomy. In Proceedings of International Conference on Research in Computational Linguistics, Taiwan.

Jones, M. and Mewhort, D. (2007). Representing word meaning and order information in a composite holographic lexicon. Psychological Review, 114(1):1-37.

Joshi, A. K., Levy, L. S., and Takahashi, M. (1975). Tree adjunct grammars. Journal of Computer and System Sciences, 10(1):136-163.

Just, M. A. and Carpenter, P. A. (1980). A theory of reading: From eye fixations to comprehension. Psychological Review, 87:329-354.

Kako, E. (1999). Elements of syntax in the systems of three language-trained animals. Animal Learning and Behavior, 27:1-14.

Kamide, Y. (2008). Anticipatory processes in sentence processing. Language and Linguistics Compass, 2(4):647-670.

Kamide, Y., Altmann, G. T., and Haywood, S. L. (2003). The time-course of prediction in incremental sentence processing: Evidence from anticipatory eye movements. Journal of Memory and Language, 49(1):133-156.

Kanejiya, D., Kumar, A., and Prasad, S. (2004). Statistical language modeling with performance benchmarks using various levels of syntactic-semantic information. In Proceedings of the 20th international conference on Computational Linguistics, pages 1161-1167, Morristown, NJ, USA. Association for Computational Linguistics. 
Katz, S. M. (1987). Estimation of probabilities from sparse data for the language model component of a speech recognizer. IEEE Transactions on Acoustics, Speech and Signal Processing, 35(3):400-401.

Keller, F., Gunasekharan, S., Mayo, N., and Corley, M. (2009). Timing accuracy of web experiments: A case study using the WebExp software package. Behavior Research Methods, 41(1):1-12.

Kennedy, A. and Pynte, J. (2005). Parafoveal-on-foveal effects in normal reading. Vision Research, 45:153-168.

Kintsch, W. (1988). The role of knowledge in discourse comprehension: a construction-integration model. Psychological Review, 95(2):163-182.

Kintsch, W. (2001). Predication. Cognitive Science, 25(2):173-202.

Kneser, R. and Ney, H. (1995). Improved backing-off for m-gram language modeling. In Proceedings of the IEEE International Conference on Acoustics, Speech and Signal Processing, pages 181-184.

Kneser, R., Peters, J., and Klakow, D. (1997). Language model adaptation using dynamic marginals. In Proceedings of EUROSPEECH 1997, pages 1971-1974.

Konieczny, L. (2000). Locality and parsing complexity. Journal of Psycholinguistic Research, 29(6):627-645.

Kuhn, R. and de Mori, R. (1990). A cache based natural language model for speech recognition. IEEE Transactions on Pattern Analysis and Machine Intelligence, 12(6):570-583.

Lakoff, G. (1977). Linguistic gestalts. In Beach, W., Fox, S., and Philosoph, S., editors, Papers from the Thirteenth Regional Meeting, Chicago Linguistic Society, pages 236-287, Chicago, Illinois. Chicago Linguistic Society. 
Landauer, T. K. and Dumais, S. T. (1996). How come you know so much? from practical problem to theory. In Hermann, D., McEvoy, C., Johnson, M., and Hertel, P., editors, Basic and applied memory: Memory in context, pages 105-126. Erlbaum, Mahwah, NJ.

Landauer, T. K., Laham, D., Rehder, B., and Schreiner, M. E. (1997). How well can passage meaning be derived without using word order: A comparison of latent semantic analysis and humans. In Nineteenth Annual Conference of the Cognitive Science Society, pages 412-417, Stanford, CA. Lawrence Erlbaum.

Landuaer, T. K. and Dumais, S. T. (1997). A solution to plato's problem: the latent semantic analysis theory of acquisition, induction and representation of knowledge. Psychological Review, 104(2):211-240.

Lapata, M. and Lascarides, A. (2003). A probabilistic account of logical metonymy. Computational Linguistics, 29(2):263-317.

Lesk, M. (1986). Automatic sense disambiguation using machine readable dictionaries: How to tell a pine cone from an ice cream cone. In Proceedings of the 5th SIGDOC, pages 24-26, New York, NY.

Levy, R. (2008). Expectation-based syntactic comprehension. Cognition, 106(3):1126-1177.

Li, Y., McLean, D., Bandar, Z., O’Shea, J., and Crockett, K. (2006). Sentence similarity based on semantic nets and corpus statistics. IEEE Transactions on Knowledge and Data Engineering, 18(8):1138-1149.

Lima, S. D. and Inhoff, A. W. (1985). Lexical access during eye fixations in reading: Effects of word-initial letter sequence. Journal of Experimental Psychology: Human Perception and Performance, 11(3):272-285. 
Lin, D. (1998). Automatic retrieval and clustering of similar words. In Proceedings of the 17th international conference on Computational linguistics, pages 768-774, Morristown, NJ, USA. Association for Computational Linguistics.

Liversedge, S. P., Paterson, K. B., and Pickering, M. J. (1998). Eye movements and measures of reading time. In Underwood, G., editor, Eye Guidance in Reading and Scene Perception, pages 55-76. Elsevier, Oxford.

Locke, J. (1690). An Essay Concerning Human Understanding.

Lowe, W. (2000). What is the dimensionality of human semantic space? In French, R. M. and Sougné, J. P., editors, Proceedings of the 6th Neural Computation and Psychology Workshop, pages 303-311, London. Springer Verlag.

Lowe, W. (2001). Towards a theory of semantic space. In Moore, J. D. and Stenning, K., editors, Proceedings of the Twenty-Third Annual Conference of the Cognitive Science Society, pages 576-581, Mahwah NJ. Lawrence Erlbaum Associates.

Lund, K. and Burgess, C. (1996). Producing high-dimensional semantic spaces from lexical co-occurrence. Behavior Research Methods, Instruments \& Computers, 28:203-208.

Marslen-Wilson, W. D. (1973). Linguistic structure and speech shadowing at very short latencies. Nature, 244(5417):522-523.

McCarthy, D., Koeling, R., Weeds, J., and Carroll, J. (2004). Finding predominant senses in untagged text. In Proceedings of the 42nd Annual Meeting of the Association for Computational Linguistics, pages 280-287, Barcelona, Spain.

McCarthy, D. and Navigli, R. (2007). Semeval-2007 task 10: English lexical substitution task. In Proceedings of the Fourth International Workshop on Semantic Evaluations (SemEval-2007), pages 48-53, Prague, Czech Republic. Association for Computational Linguistics. 
McConkie, G. W. and Rayner, K. (1975). The span of the effective stimulus during a fixation in reading. Perception and Psychophysics, 17:578-586.

McConkie, G. W. and Rayner, K. (1976). Asymmetry of the perceptual span in reading. Bulletin of the Psychonomic Society, 8:365-368.

McDonald, S. (2000). Environmental Determinants of Lexical Processing Effort. $\mathrm{PhD}$ thesis, University of Edinburgh.

McDonald, S. A. and Shillcock, R. C. (2003). Low-level predictive inference in reading: The influence of transitional probabilities on eye movements. Vision Research, $43: 1735-1751$.

Miller, G. A. and Charles, W. G. (1991). Contextual correlates of semantic similarity. Language and Cognitive Processes, 6(1).

Mitchell, J. and Lapata, M. (2008). Vector-based models of semantic composition. In Proceedings of ACL-08: HLT, pages 236-244, Columbus, Ohio. Association for Computational Linguistics.

Mitchell, J. and Lapata, M. (2009). Language models based on semantic composition. In Proceedings of the 2009 Conference on Empirical Methods in Natural Language Processing, pages 430-439, Singapore. Association for Computational Linguistics.

Mitchell, J. and Lapata, M. (2010). Composition in distributional models of semantics. Cognitive Science, 34(8):1388-1429.

Mitchell, J., Lapata, M., Demberg, V., and Keller, F. (2010). Syntactic and semantic factors in processing difficulty: An integrated measure. In Proceedings of the 48th Annual Meeting of the Association for Computational Linguistics, pages 196-206, Uppsala, Sweden. Association for Computational Linguistics.

Mnih, A. and Hinton, G. E. (2007). Three new graphical models for statistical language 
modelling. In Proceedings of the Twenty-Fourth International Conference, pages 641-648. ACM.

Moldovan, D., Clark, C., Harabagiu, S., and Maiorano, S. (2003). Cogex: A logic prover for question answering. In Proceedings of the 2003 Conference of the North American Chapter of the Association for Computational Linguistics on Human Language Technology, pages 87-93, Morristown, NJ. Association for Computational Linguistics.

Montague, R. (1974). English as a formal language. In Montague, R., editor, Formal Philosophy. Yale University Press, New Haven, CT.

Morris, R. K. (1994). Lexical and message-level sentence context effects on fixation times in reading. Journal of Experimental Psychology: Learning, Memory, and Cognition, 20:92-103.

Neville, H., Nichol, J. L., Barss, A., Forster, K. I., and Garrett, M. F. (1991). Syntactically based sentence processing classes: evidence from event-related brain potentials. Journal of Cognitive Neuroscience, 3:151-165.

Ney, H., Essen, U., and Kneser, R. (1994). On structuring probabilistic dependences in stochastic language modeling. Computer, Speech, and Language, 8:1-38.

Nunberg, G., Sag, I., and Wasow, T. (1994). Idioms. Language, 70:491-538.

Osgood, C. E., Suci, G. J., and Tannenbaum, P. H. (1957). The Measurement of Meaning. University of Illinois Press, Chicago.

Padó, S. and Lapata, M. (2007). Dependency-based construction of semantic space models. Computational Linguistics, 33(2):161-199.

Partee, B. (1995). Lexical semantics and compositionality. In Gleitman, L. and Liberman, M., editors, Invitation to Cognitive Science Part I: Language, pages 311-360. MIT Press, Cambridge, MA. 
Partee, B. (2004). Compositionality in Formal Semantics. Blackwell Publishing, Oxford, UK.

Pedersen, T., Patwardhan, S., and Michelizzi, J. (2004). WordNet::similarity - measuring the relatedness of concepts. In HLT-NAACL 2004: Demonstration Papers, pages 38-41, Boston, MA.

Phan, X.-H., Nguyen, L.-M., and Horiguchi, S. (2008). Learning to classify short and sparse text \& web with hidden topics from large-scale data collections. In $W W W$ '08: Proceeding of the 17th international conference on World Wide Web, pages 91-100, New York, NY, USA. ACM.

Pinheiro, J. C. and Bates, D. M. (2000). Mixed-effects Models in S and S-PLUS. Springer, New York.

Pinker, S. (1994). The Language Instinct: How the Mind Creates Language. HarperCollins, New York.

Plate, T. A. (1991). Holographic reduced representations: Convolution algebra for compositional distributed representations. In Mylopoulos, J. and Reiter, R., editors, Proceedings of the 12th International Joint Conference on Artificial Intelligence, Sydney, Australia, August 1991, pages 30-35, San Mateo, CA. Morgan Kaufmann.

Plate, T. A. (1993). Holographic recurrent networks. In Giles, C. L., Hanson, S. J., and Cowan, J. D., editors, Advances in Neural Information Processing Systems 5: NIPS * 92, Denver, CO, November 1992, pages 34-41, San Mateo, CA. Morgan Kaufmann.

Plate, T. A. (1995). Holographic reduced representations. IEEE Transactions on Neural Networks, 6(3):623-641.

Pollack, J. B. (1990). Recursive distributed representations. Artificial Intelligence, 46(1-2):77-105. 
Pullum, G. K. and Scholz, B. C. (2007). Systematicity and natural language syntax. Croatian Journal of Philosophy, 7(21):375-402.

Pynte, J., New, B., and Kennedy, A. (2008). On-line contextual influences during reading normal text: A multiple-regression analysis. Vision Research, 48:21722183.

Raaijmakers, J. G. W. and Schiffrin, R. M. (1981). Search of associative memory. Psychological Review, 8(2):98-134.

Rayner, K. (1998). Eye movements in reading and information processing: 20 years of research. Psychological Bulletin, 124(3):372-422.

Rayner, K. and Duffy, S. A. (1986). Lexical complexity and fixation times in reading: effects of word frequency, verb complexity, and lexical ambiguity. Memory and Cognition, 14(3):191-201.

Rayner, K., Inhoff, A. W., Morrison, R. E., Slowiaczek, M. L., and Bertera, J. H. (1981). Masking of foveal and parafoveal vision during eye fixations in reading. Journal of Experimental Psychology: Human Perception and Performance, $7(1): 167-179$.

Rayner, K. and Raney, G. E. (1996). Eye movement control in reading and visual search: Effects of word frequency. Psychonomic Bulletin and Review, 3(2):245248.

Rayner, K., Raney, G. E., and Pollatsek, A. (1995). Eye movements and discourse processing. In Lorch, R. F. and O'Brien, E. J., editors, Sources of coherence in reading, pages 9-36. Erlbaum, Hillsdale, NJ.

Rayner, K. and Well, A. D. (1996). Effects of contextual constraint on eye movements in reading: A further examination. Psychonomic Bulletin and Review, 3:504-509. 
Rayner, K., Well, A. D., Pollatsek, A., and Bertera, J. (1982). The availability of useful information to the right of fixation in reading. Perception and Psychophysics, $31: 537-544$.

Resnik, P. (1999). Semantic similarity in a taxonomy: An information-based measure and its application to problems of ambiguity in natural language. Journal of Artificial Intelligence Research, pages 95-130.

Resnik, P. and Diab, M. (2000). Measuring verb similarity. In Gleitman, L. R. and Joshi, A. K., editors, Proceedings of the 22nd Annual Conference of the Cognitive Science Society, pages 399-404, Mahwah, NJ. Lawrence Erlbaum Associates.

Richter, T. (2006). What is wrong with anova and multiple regression? analyzing sentence reading times with hierarchical linear models. Discourse Processes, 41(3):221-250.

Riggs, L. A., Merton, P. A., and Morton, H. B. (1974). Suppression of visual phosphenes during saccadic eye movements. Vision Research, 14(10):997-1011.

Roark, B. (2001). Probabilistic top-down parsing and language modeling. Computational Linguistics, 27(2):249-276.

Roark, B., Bachrach, A., Cardenas, C., and Pallier, C. (2009). Deriving lexical and syntactic expectation-based measures for psycholinguistic modeling via incremental top-down parsing. In Proceedings of the 2009 Conference on Empirical Methods in Natural Language Processing, pages 324-333, Singapore. Association for Computational Linguistics.

Rosenfeld, R. (1996). A maximum entropy approach to adaptive statistical language modeling. Computer Speech and Language, 10:187-228.

Rubenstein, H. and Goodenough, J. B. (1965). Contextual correlates of synonymy. Communications of the ACM, 8(10):627-633. 
Rudolph, S. and Giesbrecht, E. (2010). Compositional matrix-space models of language. In Proceedings of the 48th Annual Meeting of the Association for Computational Linguistics, pages 907-916, Uppsala, Sweden. Association for Computational Linguistics.

Rumelhart, D., Hinton, G., and Williams, R. (1986). Learning representations by backpropagating errors. Nature, 323(6088):533-536.

Sachs, J. S. (1967). Recognition memory for syntactic and semantic aspects of connected discourse. Perception and Psychophysics, 2:437-442.

Sachs, J. S. (1988). Memory in reading and listening to discourse. Memory and Cognition, 2:95-100.

Sahlgren, M., Host, A., and Kanerva, P. (2008). Permutations as a means to encode order in word space. In Proceedings of the 30th Annual Meeting of the Cognitive Science Society, pages 1300-1305, Mahwah, New Jersey. Lawrence Erlbaum Associates.

Salton, G., Wong, A., and Yang, C. S. (1975). A vector space model for automatic indexing. Communications of the ACM, 18(11):613-620.

Schuler, W., Miller, T., AbdelRahman, S., and Schwartz, L. (2008). Toward a psycholinguistically-motivated model of language processing. In COLING '08: Proceedings of the 22nd International Conference on Computational Linguistics, pages 785-792, Morristown, NJ, USA. Association for Computational Linguistics.

Schütze, H. (1998). Automatic word sense discrimination. Computational Linguistics, 24(1):97-124.

Simpson, G. B., Peterson, R. R., Casteel, M. A., and Burgess, C. (1989). Lexical and sentence context effects in word recognition. Journal of Experimental Psychology: Learning, Memory, and Cognition, 15:88-97. 
Smith, E. E., Shoben, E. J., and Rips, L. J. (1974). Structure and process in semantic memory: A featural model for semantic decisions. Psychological Review, 1:214241.

Smolensky, P. (1990). Tensor product variable binding and the representation of symbolic structures in connectionist systems. Artificial Intelligence, 46:159-216.

Spenader, J. and Blutner, R. (2007). Compositionality and systematicity. In Bouma, G., Krmer, I., and Zwarts, J., editors, Cognitive Foundations of Interpretation, pages 163-174. KNAW publications, Amsterdam.

Stanovich, K. E. and West, R. F. (1981). The effect of sentence context on ongoing word recognition: Tests of a two-pricess theory. Journal of Experimental Psychology: Human Perception and Performance, 7(3):658-672.

Stenning, K. and van Lambalgen, M. (2008). Human reasoning and cognitive science. MIT, Cambridge, MA.

Stolcke, A. (1995). An efficient probabilistic context-free parsing algorithm that computes prefix probabilities. Computational Linguistics, 21(2):165-201.

Stolcke, A. (2002). SRILM - an extensible language modeling toolkit. In Proceedings of the Internatinal Conference on Spoken Language Processing, Denver, Colorado.

Sturt, P. and Lombardo, V. (2005). Processing coordinated structures: Incrementality and connectedness. Cognitive Science, 29(2):291-305.

Tanenhaus, M. K., Spivey-Knowlton, M. J., Eberhard, K. M., and Sedivy, J. C. (1995). Integration of visual and linguistic information in spoken language comprehension. Science, 268(5217):1632-1634.

Thompson, C. A., Mooney, R. J., and Tang, L. R. (1997). Learning to parse natural language database queries into logical form. In Proceedings of the ICML-97 Workshop on Automata Induction, Grammatical Inference, and Language Acquisition. 
van Berkum, J. J. A., Brown, C. M., and Hagoort, P. (1999). Early referential context effects in sentence processing: Evidence from event-related brain potentials. Journal of Memory and Language, (41):147-182.

Vapnik, V. (1995). The Nature of Statistical Learning Theory. Springer, New York.

Vitu, F., McConkie, G. W., Kerr, P., and ORegan, J. K. (2001). Fixation location effects on fixation durations during reading: an inverted optimal viewing position effect. Vision Research, 41:3513-3533.

Vitu, F., McConkie, G. W., and Zola, D. (1998). About regressive saccades in reading and their relation to word identification. In Underwood, G., editor, Eye Guidance in Reading and Scene Perception, pages 101-124. Elsevier, Oxford.

Wallach, H. M. (2006). Topic modeling: beyond bag-of-words. In Proceedings of the 23rd International Conference on Machine Learning, pages 977-984, New York, NY, USA. ACM.

Wandmacher, T. and Antoine, J.-Y. (2007). Methods to integrate a language model with semantic information for a word prediction component. In Proceedings of the 2007 Joint Conference on Empirical Methods in Natural Language Processing and Computational Natural Language Learning, pages 506-513, Prague, Czech Republic. Association for Computational Linguistics.

Weeds, J. (2003). Measures and Applications of Lexical Distributional Similarity. $\mathrm{PhD}$ thesis, Department of Informatics, University of Sussex.

Weiss, S. M. and Kulikowski, C. A. (1991). Computer Systems that Learn: Classification and Prediction Methods from Statistics, Neural Nets, Machine Learning, and Expert Systems. Morgan Kaufmann, San Mateo, CA.

West, R. F. and Stanovich, K. E. (1986). Robust effects of syntactic structure on visual word processing. Journal of Memory and Cognition, 14:104-112. 
Widdows, D. (2008). Semantic vector products: Some initial investigations. In Proceedings of the Second Symposium on Quantum Interaction (QI-2008), Oxford, UK. College Publications.

Wittgenstein, L. (1953). Philosophical Investigations. Blackwell Publishers.

Wu, S. (2010). Vectorial Representations of Meaning for a Computational Model of Language Comprehension. $\mathrm{PhD}$ thesis, University of Minnesota.

Yamada, I., Torisawa, K., Kazama, J., Kuroda, K., Murata, M., De Saeger, S., Bond, F., and Sumida, A. (2009). Hypernym discovery based on distributional similarity and hierarchical structures. In Proceedings of the 2009 Conference on Empirical Methods in Natural Language Processing, pages 929-937, Morristown, NJ. Association for Computational Linguistics.

Zhang, R. and Rudnicky, A. I. (2002). Improve latent semantic analysis based language model by integrating multiple level knowldege. In Proceedings of the 7th International Conference on Spoken Language Processing, pages 893-897, Denver, $\mathrm{CO}$. 